UNIVERSITÉ DU QUÉBEC À RIMOUSKI

\title{
L'INFLUENCE DU CLAVARDAGE SUR LA MAÎTRISE DU FRANÇAIS ÉCRIT DES ÉLÈVES DU PREMIER CYCLE DU SECONDAIRE
}

\author{
Mémoire présenté \\ dans le cadre du programme de maîtrise en éducation \\ en vue de l'obtention du grade de maître ès arts \\ PAR \\ MARIE-EVE GONTHIER
}

Octobre 2011 


\title{
UNIVERSITÉ DU QUÉBEC À RIMOUSKI
}

Service de la bibliothèque

\begin{abstract}
Avertissement
La diffusion de ce mémoire ou de cette thèse se fait dans le respect des droits de son auteur, qui a signé le formulaire «Autorisation de reproduire et de diffuser un rapport, un mémoire ou une thèse ». En signant ce formulaire, l'auteur concède à l'Université du Québec à Rimouski une licence non exclusive d'utilisation et de publication de la totalité ou d'une partie importante de son travail de recherche pour des fins pédagogiques et non commerciales. Plus précisément, l'auteur autorise l'Université du Québec à Rimouski à reproduire, diffuser, prêter, distribuer ou vendre des copies de son travail de recherche à des fins non commerciales sur quelque support que ce soit, y compris l'Internet. Cette licence et cette autorisation n'entraînent pas une renonciation de la part de l'auteur à ses droits moraux ni à ses droits de propriété intellectuelle. Sauf entente contraire, l'auteur conserve la liberté de diffuser et de commercialiser ou non ce travail dont il possède un exemplaire.
\end{abstract}


Composition du jury :

Bastien Sasseville, président du jury, Université du Québec à Rimouski

Stéphanie Leblanc, directrice de recherche, Université du Québec à Rimouski

Jean-Yves Lévesque, correcteur, Université du Québec à Rimouski

Diane Huot, examinatrice externe, Université Laval

Dépôt initial le 13 mai 2011

Dépôt final le 11 octobre 2011 


\section{REMERCIEMENTS}

Je remercie toutes les personnes qui ont participé de près ou de loin à l'aboutissement de la rédaction de ce mémoire. Tout d'abord, je remercie ma directrice de recherche, Stéphanie Leblanc, pour son aide tout au long de la rédaction et pour ses conseils avisés. Ses suggestions m'ont été précieuses et m'ont permis de construire un mémoire correspondant à mon souci d'excellence. Sa grande disponibilité est également à souligner. Madame Leblanc m'a transmis son intérêt envers la recherche, ce qui fait en sorte que je me dirige vers le doctorat pour la prochaine année.

Également, je ne peux passer sous silence l'aide financière accordée par le Conseil de recherches en sciences humaines du Canada (CRSH), qui m'a accordé une bourse de maîtrise Joseph-Armand-Bombardier pour l'année scolaire 2010-2011. Cette bourse m'a permis de me consacrer à temps plein à mon mémoire de maîtrise lors de ma seconde année d'étude, ce qui fait en sorte que je peux aujourd'hui déposer dans les délais prévus.

L'implication de la commission scolaire et de l'école secondaire ayant accepté de collaborer à mon projet se doit également d'être mentionnée. Celles-ci, dont les noms ne peuvent être cités par souci de confidentialité, ont accepté avec ouverture de participer à ce projet, ce qui a été très apprécié. Je tiens aussi à souligner la collaboration des trois enseignants qui m'ont ouvert les portes de leur classe et qui ont intégré leurs groupes à la recherche. Sans eux, ce projet n'aurait pu être mené à terme. Je dois aussi soulever l'accueil exemplaire de la directrice adjointe de l'école. La collaboration des élèves, qui se sont soumis avec intérêt à mes activités et qui semblaient contents de prendre une part active à une recherche de niveau universitaire, a également contribué au plaisir que j'ai eu à réaliser cette recherche. 
De plus, j'adresse mes remerciements aux professeurs du département des sciences de l'éducation de l'Université du Québec à Rimouski. Les échanges lors des cours de maîtrise, les aspects théoriques de la recherche, ainsi que les recommandations avisées de plusieurs d'entre eux, autant lors des cours qu'à l'extérieur de ceux-ci, ont su développer chez moi une véritable passion pour la recherche. Les conseils de mes collègues de maîtrise ont aussi favorisé le développement de mon projet.

Par ailleurs, je remercie madame Paule Maranda, qui s'est chargée de la révision linguistique et de la mise en page finale de ce mémoire.

Mon amoureux, Jérôme, a su m'apporter son support tout au long de mes deux années de maîtrise. Il a toujours fait preuve d'intérêt quant à mon sujet de recherche et m'a écouté parler maintes fois de mon mémoire, de mes idées et de mes interrogations. Il a toujours pris le temps de me conseiller et de me rassurer en cas de besoin. Il n'aurait pu être un partenaire plus à l'écoute.

Enfin, je tiens à mentionner le soutien exemplaire et l'encouragement de ma famille. Les membres de ma famille se sont régulièrement informés de l'évolution de ma recherche et de l'écriture de mon mémoire; ils seront parmi les premiers à me lire. 


\section{AVANT-PROPOS}

Lors des dernières années, j'ai eu l'occasion d'agir comme enseignante en adaptation scolaire et sociale dans des groupes de niveau secondaire. En ma qualité d'enseignante, j'ai été étonnée lorsque j'ai constaté pour la première fois, dans les écrits de certains de mes élèves, la présence de mots qui semblaient correspondre au code écrit attribué au clavardage. Par exemple, un élève, à défaut d'écrire le groupe de mots parce que, avait tout simplement écrit $p c q$; il avait donc omis des lettres, afin d'en faire un seul mot. J'en suis ainsi venue à me poser un certain nombre de questions. Notamment, je me suis demandé s'il s'agissait d'une action volontaire, dans le but de sauver du temps et de remettre sa copie plus rapidement. Je me suis aussi interrogée à savoir si cet élève avait tout simplement été inattentif et n'avait pas pris conscience de sa méprise. Ce dernier point serait davantage inquiétant, puisque cela laisserait entendre qu'il est plausible que le clavardage ait une influence défavorable sur la maîtrise de la langue française écrite. En effet, la coupure de lettres constitue l'une des caractéristiques principales de l'écriture codée associée au clavardage, que j'ai eu l'occasion d'étudier rigoureusement dans le cadre de la réalisation de ce mémoire.

D'ailleurs, j'ai eu l'occasion, lors de mes années de pratique, d'interroger informellement des enseignants de français en classe régulière et en classe d'adaptation scolaire ayant plusieurs années d'expérience derrière eux. Ceux-ci m'ont fait part de leur vision personnelle concernant les possibles répercussions du clavardage et du fait qu'ils ont détecté des mots étant reliés au code de la cyberlangue (en tk, pkoi, pcq...) dans les productions écrites de leurs élèves. Ces expériences, discussions et opinions, ainsi que le fait que plusieurs articles ont été rédigés sur le sujet dans des journaux ou des revues d'actualité, sans toutefois s'y attarder d'un point de vue scientifique avec la clientèle ciblée pour les besoins de ce mémoire, suggèrent qu'il y a matière à investiguer la question, d'où l'intérêt de la présente recherche. 


\section{RÉSUMÉ}

Les jeunes d'aujourd'hui passent de plus en plus de temps à s'adonner au clavardage. Étant donné l'usage fréquent de l'ordinateur à des fins de communication et les caractéristiques particulières de la langue associée au clavardage, il est pertinent de réaliser une recherche visant à vérifier l'influence du clavardage sur la maîtrise du français écrit. C'est pourquoi la question de recherche soulevée dans le cadre de ce mémoire est la suivante: le clavardage a-t-il une influence sur la maîtrise du français écrit des élèves du premier cycle du secondaire?

Dans le but de répondre à cette question, l'hypothèse principale suppose que le clavardage a une influence défavorable sux la maîtrise du français écrit des élèves du premier cycle du secondaire. Afin de la vérifier, trois hypothèses issues de celle-ci ont été vérifiées, en comparant les erreurs de français associées au clavardage avec l'âge auquel les jeunes débutent à clavarder, le temps hebdomadaire consacré au clavardage, ainsi que le fait que l'élève clavarde ou non.

La méthodologie utilisée est de nature quantitative et est constituée de trois différentes étapes, soit des séances d'observations sur les sites francophones de clavardage, la réalisation d'une dictée intégrant les mots puisés lors de ces séances d'observations, de même que la passation d'un questionnaire sur les habitudes informatiques des sujets. L'analyse des données a été effectuée par l'intermédiaire de tests statistiques visant à déterminer s'il y a des liens significatifs entre les diverses variables. Cette recherche a été réalisée avec la participation de 158 élèves du premier cycle du secondaire.

Les résultats ont démontré que le clavardage n'a pas d'influence défavorable sur la maîtrise du français écrit des élèves de ce niveau. En effet, d'après nos analyses, nous avons constaté que les trois variables mesurant le clavardage n'ont pas d'incidence sur le nombre d'erreurs de français relevé dans la dictée. Il est toutefois nécessaire de demeurer prudents dans la généralisation de ces résultats puisque ceux-ci peuvent différer selon les échantillons d'élèves.

En somme, cette recherche apporte à la communauté des réponses concernant l'influence de ce média sur la maîtrise de la langue française. Notamment, les enseignants, qui constatent la présence de mots associés au clavardage dans les écrits de leurs élèves, de même que les parents, qui sont outrés et se questionnent lorsqu'ils observent leurs enfants clavarder, savent maintenant que l'essor de ce nouveau mode de communication n'est pas à craindre.

Mots-clés : clavardage, Internet, maîtrise du français écrit, adolescents. 


\begin{abstract}
Youngsters today spend more and more time chatting. Considering the frequency with which they use the computer for communication purposes and the particular characteristics of the language associated with online chatting, conducting research to verify the influence chatting has on written French proficiency seems quite relevant. This is why this memoire's research delves into the following question: Does chatting influence students' proficiency with written French during the first cycle of high school?

In order to answer this question, the primary assumption supposes that chatting has a negative influence on students' proficiency with written French during the first cycle of high school. To verify this, three secondary assumptions relative to the latter have been investigated, namely, to compare the level of French errors associated with chatting in reference to the age at which the youngster began to chat, the amount of time spent chatting over the course of one week and whether or not the student chats.
\end{abstract}

The methodology used is of quantitative nature and includes three different steps, namely observation sessions on French chatting sites, a dictation that integrates words drawn from these observation sessions, as well as a questionnaire on the subjects' computer habits. Data analysis was performed using statistic tests aimed at establishing whether or not the relationship between these variables is significant. This research was realized with the participation of 158 students in their first cycle of high school.

Results show that chatting does not have a negative influence on the proficiency of students of this level to write proper French. In fact, according to our analyses, we have observed no incidence on the number of French errors associated with chatting in relation to either of the variables analyzed. It is however necessary to be careful in the generalization of these results because they can differ according to the students.

Finally, this research provides the community with answers concerning the influence this media has on French language proficiency. Basically, teachers who observe the presence of words associated with chatting in their students' texts or parents who become outraged or question their kids' abilities when they see them chat, can now be relieved to know that this rapidly growing means of communication is not to be feared.

Key words : chatting, Internet, written French proficiency, teenagers. 


\section{TABLE DES MATIĖRES}

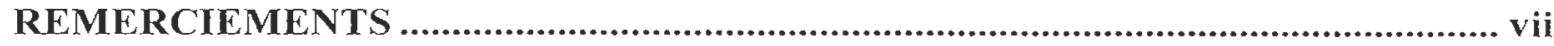

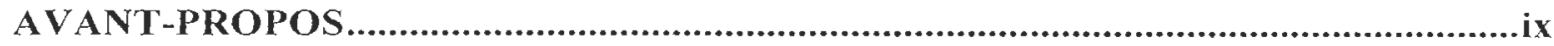

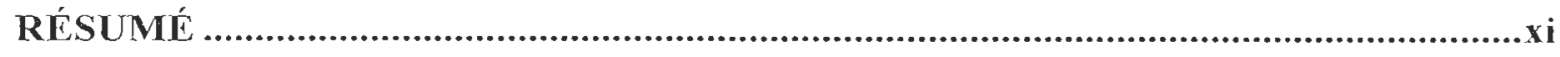

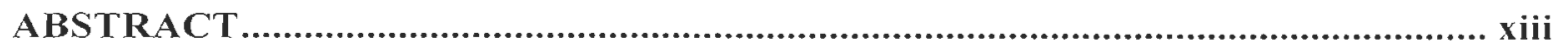

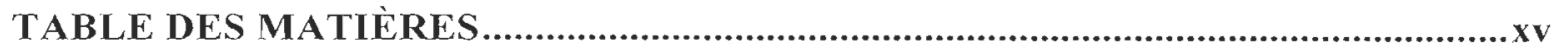

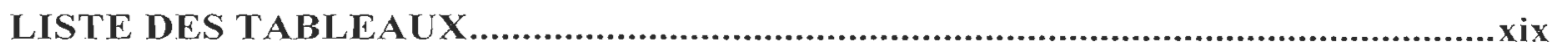

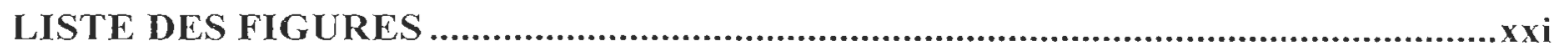

LISTE DES ABRÉVIATIONS, DES SIGLES ET DES ACRONYMES ............... xxiii

CHAPITRE 1 INTRODUCTION GÉNÉRALE ..................................................

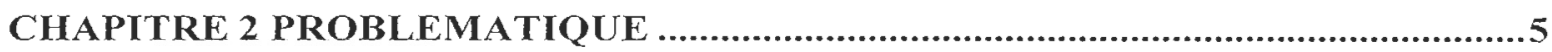

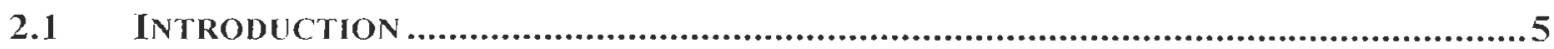

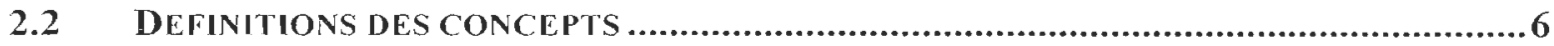

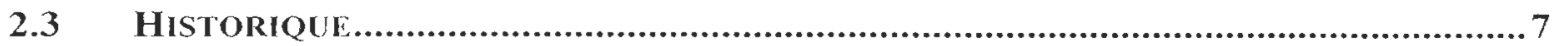

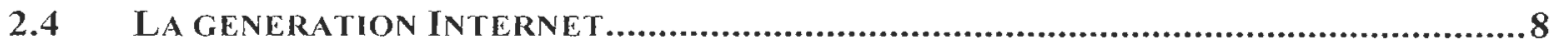

2.5 ASPECTS POSITIFS ET NEGATIFS DU CLAVARDAGE ....................................11

2.6 CARACTERISTIQUES PRINCIPALES DE LA LANGUE ECRITE CODEE UTILISEE LORS

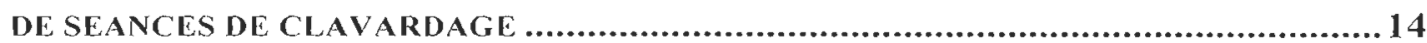

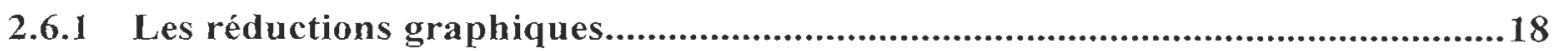

2.6.2 Les réductions et transformations avec variantes phonétiques .......................19

2.6.3 Particularités de ce mode de communication .....................................................20

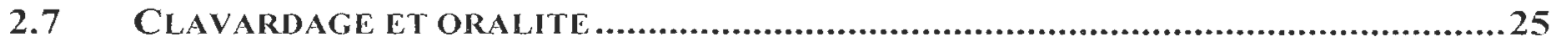

2.8 OPINIONS SUR L'EFFET DU CLAVARDAGE SUR LA MAITRISE DE LA LANGUE

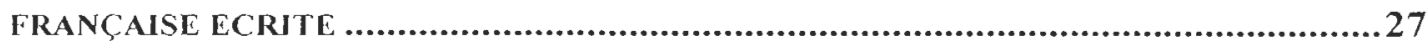

2.9 ÉTUDES SUR L'INFLUENCE DU CLAVARDAGE SUR LA LANGUE ...............................33

2.10 DIFFERENCES ENTRE LES SEXES AU NIVEAU DES PERFORMANCES EN ECRITURE ...37

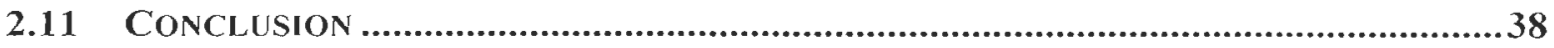

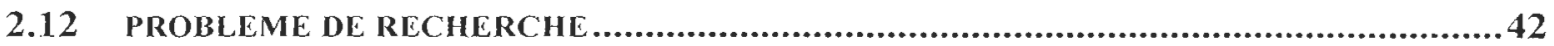




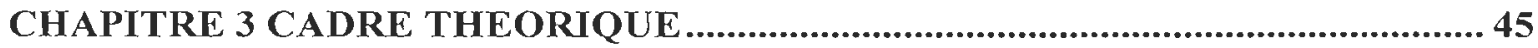

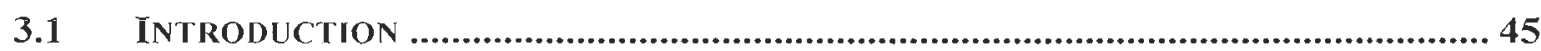

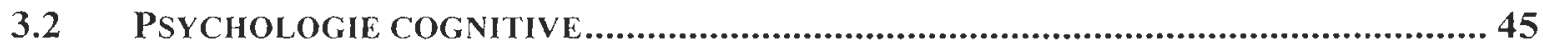

3.3 STRATEGIES METACOGNITIVES ET COGNITIVES............................................... 47

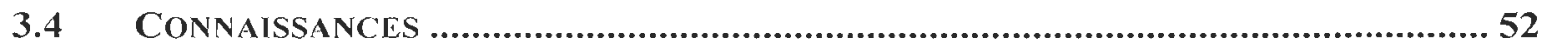

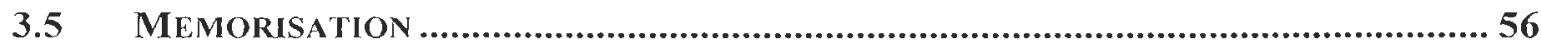

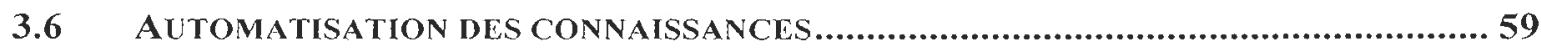

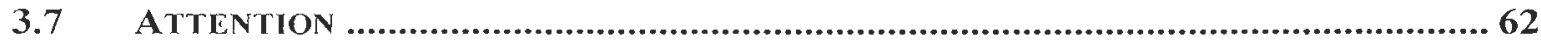

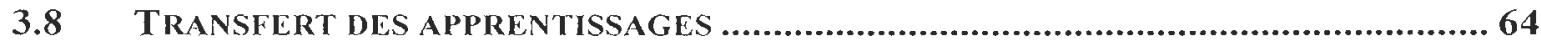

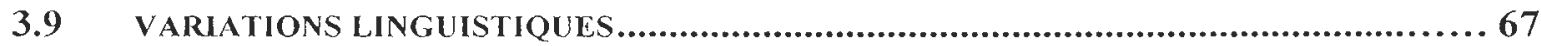

3.10 LE RECOURS AU VERLAN ET LA MAITRISE DE LA LANGUE FRANÇAISE ORALE ...... 72

3.11 ÉCRITURE ET STRATEGIES D'ECRITURE..................................................... 74

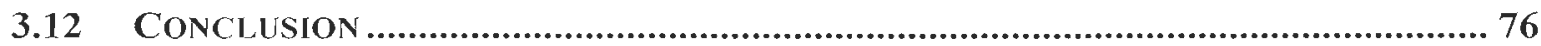

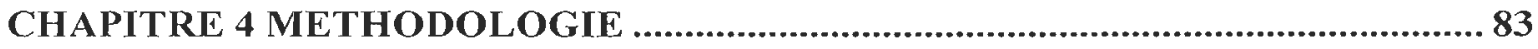

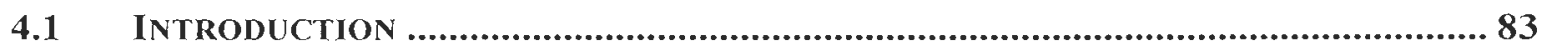

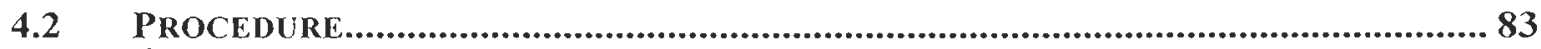

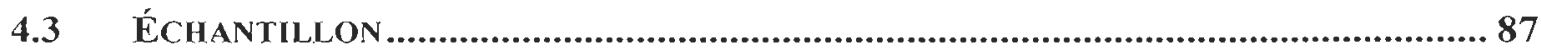

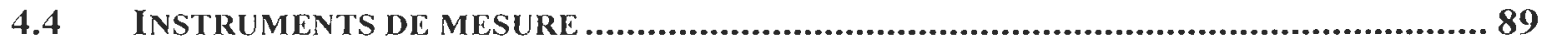

4.4.1 Séances d'observations sur les sites consacrés au clavardage ........................... 89

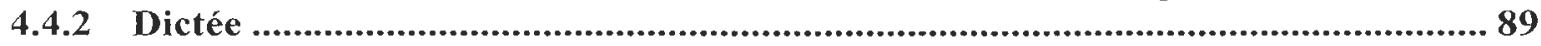

4.4.3 Questionnaire sur les habitudes informatiques ............................................. 92

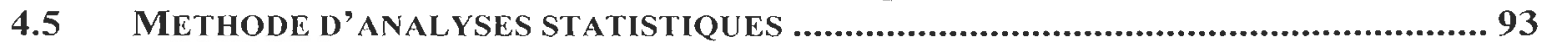

4.5.1 Méthodologie et recherche quantitative ........................................................ 93

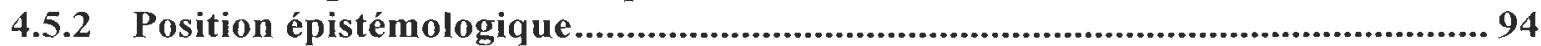

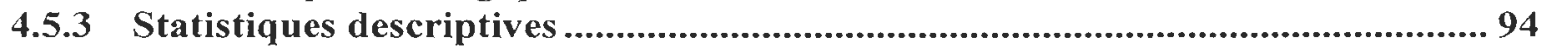

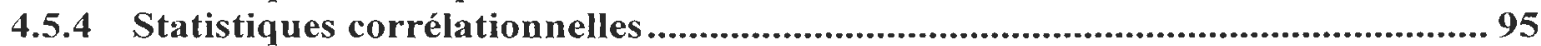

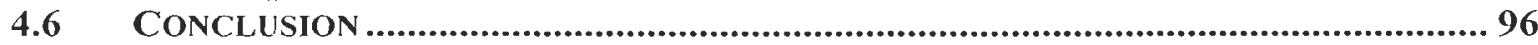

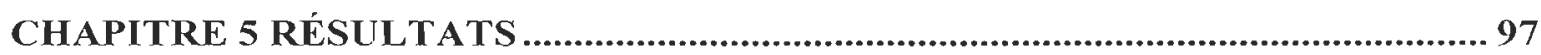

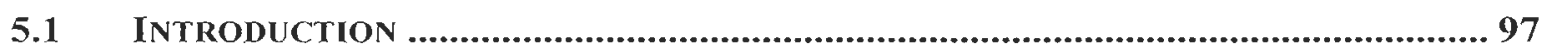

5.2 QUESTION DE RECHERCHE ET HYPOTHESES ................................................... 97

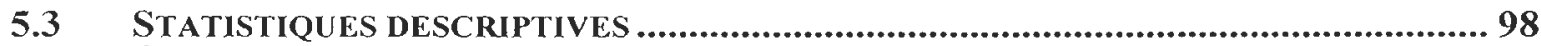

5.3.1 Âge de début du clavardage................................................................. 98

5.3.2 Temps hebdomadaire consacré au clavardage ....................................... 100

5.3 .3 Le fait que l'élève clavarde ou non ........................................................ 102

5.3.4 Erreurs de français associées au clavardage ................................................... 103

5.4 STATISTIQUES CORRELATIONNELLES ............................................................ 104

5.4.1 Lien entre l'âge de début du clavardage et les erreurs de français associées

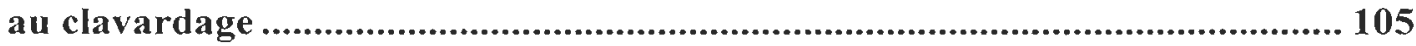

5.4.2 Lien entre le temps hebdomadaire consacré au clavardage et les erreurs de français associées au clavardage 
5.4.3 Lien entre le fait que l'élève clavarde ou non et les erreurs de français associées au clavardage.........................................................................................108

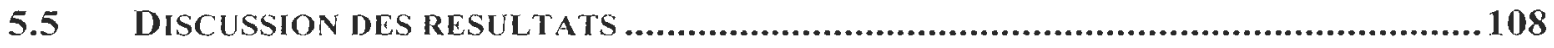

CHAPITRE 6 CONCLUSION GÉNÉRALE...............................................115

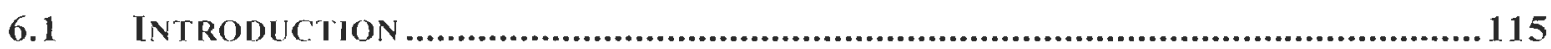

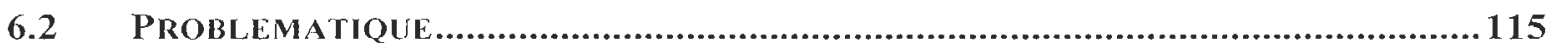

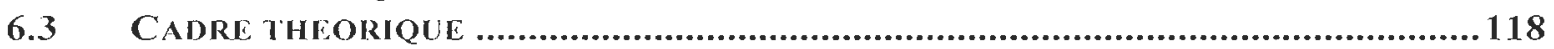

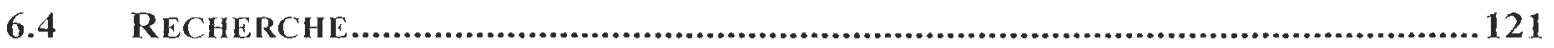

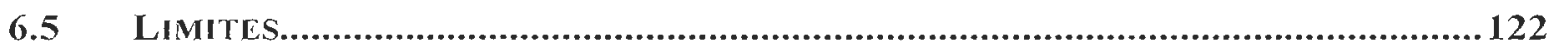

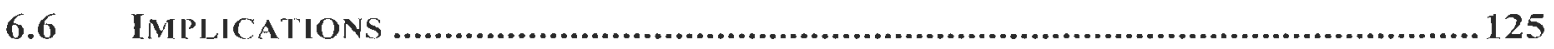

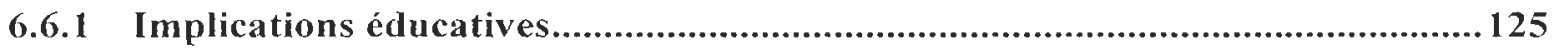

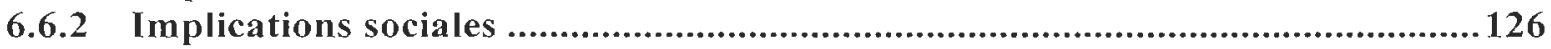

6.6.3 Implications scientifiques et avenues de recherches .................................... 128

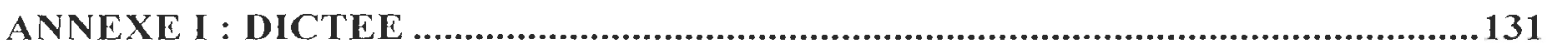

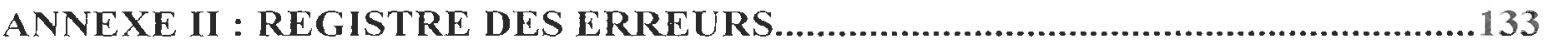

ANNEXE III : QUESTIONNAIRE SUR LES HABITUDES INFORMATIQUES...139

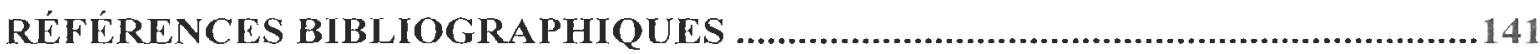


xviii 


\section{LISTE DES TABLEAUX}

Tableau 1: Les formes linguistiques associées au clavardage .................................... 15

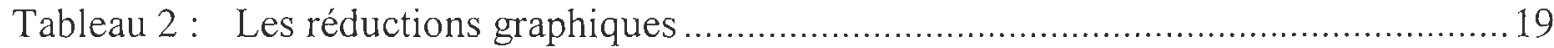

Tableau 3: Les réductions et transformations avec variantes phonétiques.....................20

Tableau 4: Particularités de ce mode de communication .........................................21

Tableau 5: Principales caractéristiques des sujets de l'échantillon .............................88

Tableau 6: Mots inclus dans le registre des erreurs ............................................. 134

Tableau 7: Groupes de mots inclus dans le registre des erreurs ........................... 136 


\section{LISTE DES FIGURES}

Figure 1: Âge de début du clavardage ........................................................... 99

Figure 2: Temps hebdomadaire consacré au clavardage ....................................... 102

Figure 3: Erreurs de français associées au clavardage ............................................. 103

Figure 4: Erreurs de français associées au clavardage selon l'âge de début du

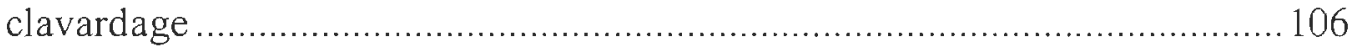

Figure 5: Erreurs de français associées au clavardage selon le temps hebdomadaire

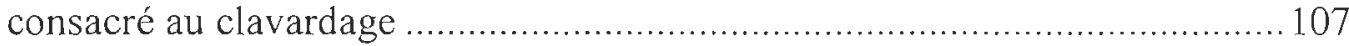




\title{
LISTE DES ABRÉVIATIONS, DES SIGLES ET DES ACRONYMES
}

\author{
Chat : Clavardage \\ ICQ : I seek you \\ IRC : Internet Relay Chat \\ MSN : Microsoft Network \\ SMS : Short message service (textos)
}


xxiv 


\section{CHAPITRE 1}

\section{INTRODUCTION GÉNÉRALE}

Le clavardage est une pratique très populaire au Québec et dans plusieurs autres pays. De nombreux adolescents le préconisent comme outil de conversation. Par le biais de ce récent mode de communication, les jeunes francophones transgressent les normes de la langue française, puisqu'ils utilisent une langue codée qui est très différente du français conventionnel. Cette recherche a pour objectif de vérifiel l'influence du clavardage sur la maîtrise du français écrit des élèves du premier cycle du secondaire, et ce, au regard des particularités de la langue codée associée au clavardage et de l'influence de la psychologie cognitive sur l'apprentissage.

Quelques études anglophones se sont attardées au phénomène du clavardage. Notamment, selon l'étude de Varnhagen, McFall, Pugh, Routhledge et Sumida-MacDonald (2009), le clavardage n'a pas d'influence sur la maîtrise de la langue anglaise des adolescents de 12 à 17 ans. En revanche, cette recherche était basée sur des tests d'épellation, ce qui diffère complètement des épreuves d'écriture. En effet, cette étude n'a donc pas vérifié l'influence du clavardage sur la maîtrise de la langue écrite. Du côté francophone, les connaissances sur le phénomène sont davantage du domaine des opinions. Effectivement, peu d'études se sont penchées sur la question. À notre connaissance, une seule recherche a étudié l'impact du clavardage sur la langue écrite au Québec. Il s'agit de l'étude de Lafontaine, Blouin-Bradette, Cantin-Fontaine et Fortier (2005), qui a conclu que le clavardage n'affecte pas la langue française écrite des élèves de cinquième secondaire, en comparant des tâches écrites requérant les mots codés du clavardage des clavardeurs et des non-clavardeurs. Par contre, la clientèle et la méthode ne sont pas les mêmes que pour la

présente recherche, de même que les différentes variables ciblées, d'oủ la contribution 
originale de celle-ci. De plus, de toute évidence, une seule étude sur le clavardage et son impact sur la langue française n'est pas suffisante pour le domaine éducatif, ce qui ajoute à la nécessité de mener une telle recherche sur le sujet.

D'ailleurs, il s'agit d'une problématique d'actualité, étant donné que nous savons que de nombreux jeunes clavardent quotidiennement. Nous pouvons également remarquer qu'il s'agit d'un phénomène qui se retrouve au cœur des débats, puisque plusieurs articles ont traité du thème à l'étude, sans toutefois déterminer scientifiquement l'effet du clavardage sur la langue écrite. Ces éléments rappellent la pertinence de centrer une étude sur le sujet. C'est pourquoi la question de recherche relative à ce mémoire de maîtrise se pose comme suit: le clavardage a-t-il une influence sur la maîtrise du français écrit des élèves du premier cycle du secondaire? Dans le but de répondre à cette question, l'hypothèse principale propose que le clavardage a une influence défavorable sur la maîtrise du français écrit des élèves du premier cycle du secondaire.

Le chapitre deux, qui fait suite à cette introduction, est centré sur la problématique et consiste en une recension des écrits concernant le sujet étudié. Cette recension se concentre principalement sur les caractéristiques de la langue couramment utilisée en situation de clavardage, sur les opinions des acteurs concernés par la question, ainsi que sur les différentes études réalisées sur le clavardage lors des années antérieures.

Ensuite, le chapitre trois présente le cadre théorique, qui utilise la psychologie cognitive et la théorie du traitement de l'information afin d'analyser le phénomène à l'étude. Il est nécessaire, dans le but de cerner les mécanismes de l'influence du clavardage sur la maîtrise du français écrit, de comprendre comment réfléchit l'élève au niveau cognitif, autant lorsqu'il est en processus de rédaction en contexte scolaire qu'en situation de clavardage. Divers aspects de la psychologie cognitive sont détaillés en lien avec l'objet de cette recherche, tels que la métacognition, le transfert des apprentissages et les variations linguistiques. Les liens établis entre le clavardage et cette forme de psychologie constituent aussi un aspect original de ce mémoire, étant donné que le clavardage est abordé selon ce point de vue, ce qui est nouveau et n'avait pas encore été investigué à ce jour. 
Le chapitre quatre aborde les aspects méthodologiques de cette étude. Principalement, la méthodologie est de nature quantitative et selon une perspective positiviste. Les variables âge de début du clavardage, temps hebdomadaire consacré au clavardage et le fait que l'élève clavarde ou non ont été recueillies par l'intermédiaire d'un questionnaire sur les habitudes informatiques. De même, les erreurs de français associées au clavardage ont été quant à elles mesurées à l'aide d'une dictée. La cueillette de ces données a permis de connaître de nouvelles statistiques autant descriptives que corrélationnelles concernant le phénomène, qui sont à ce jour peu connues. Il importe de mentionner que 158 élèves, répartis dans sept groupes du premier cycle du secondaire, ont participé à cette recherche.

Par la suite, le cinquième chapitre expose les résultats obtenus, qui sont ensuite discutés. L'hypothèse principale, qui suppose que le clavardage a une influence défavorable sur la maîtrise du français écrit des élèves du premier cycle du secondaire, a été vérifiée avec l'appui de trois autres hypothèses. Les tests statistiques utilisés en conséquence visaient à vérifier s'il y a un lien significatif entre les erreurs de français associées au clavardage et l'âge de début du clavardage, le temps hebdomadaire consacré au clavardage et le fait que l'élève clavarde ou non.

Pour terminer, la conclusion rappelle les éléments principaux de chaque partie du mémoire. Elle fait aussi mention des limites de la recherche, des implications éducatives, sociales et scientifiques de celle-ci, ainsi que des pistes de recherches futures.

En somme, cette étude permettra à la communauté de savoir si le clavardage a une influence défavorable sur la maîtrise de la langue française écrite. Les divers articles consultés et opinions recueillies permettent de constater que la réalisation d'une telle recherche est nécessaire, compte tenu de la popularité en effervescence de ce mode de communication et du peu de connaissances que nous avons jusqu'à maintenant de son impact sur la langue. 



\section{CHAPITRE 2}

\section{PROBLÉMATIQUE}

\subsection{INTRODUCTION}

Depuis plusieurs années, les communications ont subi des changements importants. Les gens explorent d'autres avenues que les lettres écrites à la main, les conversations téléphoniques et les discussions orales entre personnes réunies dans un même lieu. L'avènement de l'informatique et des nouvelles technologies fait en sorte que d'autres options sont aujourd'hui offertes à l'ensemble des individus. Ceux-ci ont maintenant la possibilité de communiquer par le biais du clavardage, c'est-à-dire par des discussions virtuelles en direct où les gens discutent avec les personnes de leur choix. Le clavardage est le thème à la base de la présente recherche, dont le but est de vérifier si son utilisation a une influence sur la maîtrise du français écrit des jeunes du premier cycle du secondaire. La problématique est divisée en huit parties. Dans ce chapitre figurent une définition des concepts, un bref historique concernant l'avènement de l'informatique, ainsi qu'une partie définissant les caractéristiques de la clientèle associée à ce mémoire, soit les adolescents entre 12 et 16 ans. Par ailleurs, il est pertinent de faire ressortir les aspects positifs et négatifs de ce média récent, de même que les liens qui ont été ciblés entre le clavardage et la langue orale. De plus, les statistiques quant à l'utilisation de ce nouveau mode de communication, ses principales caractéristiques, qui sont sensiblement les mêmes que celles des textos, les opinions des acteurs de l'éducation ainsi que les études menées sur le sujet seront abordées. 


\subsection{DEFINITIONS DES CONCEPTS}

Les termes clavardage (issu de clavier et bavardage), bavardage clavier et cyber bavardage ont été nommés par l'Office québécois de la langue française (1997) pour désigner ce nouveau phénomène. Selon cet organisme, le clavardage est défini comme étant une " activité permettant à un internaute d'avoir une conversation écrite, interactive et en temps réel avec d'autres internautes, par clavier interposé » (Office québécois de la langue française, 1997). Les discussions par le biais du clavardage se font par l'intermédiaire de sites Internet (bavardoirs) ou de programmes comme Msn Messenger. Les individus se servent également des réseaux sociaux pour clavarder; Facebook et Myspace sont devenus des outils de clavardage très appréciés des internautes. En somme, le clavardage peut être défini comme une forme de bavardage en ligne (discussion virtuelle, discussion en direct, messagerie instantanée, discussion virtuelle, chat) où la langue codée y est la langue associée. Le terme privilégié dans le cadre de cette recherche pour désigner le phénomène est clavardage et le code reconnu pour en désigner son code écrit sera la langue codée. Des personnes d'âges variés et du monde entier sont touchées par le phénomène du clavardage, malgré le fait que les adolescents en sont les plus grands adeptes (Paré, 2001). En effet, plusieurs d'entre eux passent de nombreuses heures par jour à clavarder sur des sites Internet et sur des programmes ayant cette fonction. De même, les textos, cette nouvelle technologie où les individus peuvent s'envoyer des messages par le biais de leur téléphone cellulaire, sont aussi particulièrement populaires. Ce dernier terme (texto) sera employé lorsque les messages de ce type seront abordés. D'ailleurs, les utilisateurs des textos recourent aussi à la langue codée, ce qui fait en sorte que la majorité des caractéristiques de la langue codée sont les mêmes pour le clavardage et pour les textos. Un autre terme permet l'appellation des textos : il s'agit des SMS (short message service). Le clavardage demeure le principal phénomène à l'étude dans le cadre de ce mémoire, bien que les textos et le courrier électronique, des modes de communication connexes, seront aussi abordés en recension des écrits. En effet, les internautes ont la possibilité de communiquer par courrier électronique (mail, courriel), qui constitue un service d'envoi de messages électroniques se 
réalisant par l'intermédiaire d'Internet. La langue codée est un peu moins présente dans le courrier électronique. Le terme courrier électronique sera préconisé dans cette recherche pour désigner ce mode de transmission de messages.

\subsection{HistoriQue}

Dans le cadre de l'actuelle recherche, un bref historique du clavardage permettra de mieux comprendre l'origine de ce mode de communication en pleine effervescence.

Internet a vu le jour en 1969 et a été développé par le département américain de la défense. À vrai dire, plusieurs centres scientifiques militaires, publics et privés ont contribué à sa création. Suite à la conception de ce nouveau média qui a révolutionné complètement la circulation de l'information dans le monde, le courrier électronique est né en 1971, année lors de laquelle deux programmes de cette nature ont été créés par Tomlinson. Par contre, les programmes de courrier électronique ont été normalisés plusieurs années plus tard, en 1977. En 1979, les forums de discussion ont été mis au point, utilisables par l'intermédiaire du réseau «Usenet». Par la suite, dans le but d'offrir un moyen fonctionnel de transmission de l'information, le "World Wide Web » a été conçu en 1984 (Marcoccia, 2003). IRC (Internet Relay Chat) a été quant à lui inventé en 1988 et constitue le premier système de clavardage sur Internet. Il s'agit de pages web où plusieurs personnes communiquent entre elles. Leurs commentaires sont alors à la vue de tous, autant les utilisateurs que les visiteurs. IRC a suscité rapidement l'intérêt des internautes, ce qui fait en sorte que ce logiciel a été très populaire dès son entrée dans le domaine informatique. Par la suite, des systèmes de clavardage permettant la communication en duo ont également été mis au point. ICQ (I seek you) a été créé en 1996 et constitue l'un des premiers programmes de clavardage permettant la communication en dyade (Fuks, Pimentel et De Lucena, 2006). Suite à l'apparition d'ICQ, d'autres logiciels ont été créés, par exemple Msn Messenger, qui est toujours très populaire en temps actuel (Bouffard et Pouliot, 2007). D'ailleurs, il est maintenant possible de clavarder avec un nombre de 
personnes illimitées. Selon Bouffard et Pouliot (2007), le nombre d'utilisateurs du clavardage se compte à 360 millions dans le monde entier, et ce nombre serait en constante augmentation. De nos jours, approximativement $74 \%$ des jeunes utilisent le clavardage pour communiquer selon ces auteurs.

\subsection{LA GENERATION INTERNET}

Il est de circonstance de faire ressortir quelques caractéristiques communes aux adolescents d'aujourd'hui à l'égard de l'utilisation qu'ils font de l'informatique, cela dans le but de se familiariser avec leur réalité.

La clientèle de jeunes étudiée par l'intermédiaire de ce mémoire est en quelque sorte née avec Internet. Paré (2001) a intitulé cette génération comme étant justement la génération Internet. Cette expression distingue bien les adolescents d'aujourd'hui de ceux des précédentes générations, étant donné qu'Internet est au centre de leur vie. En effet, ils utilisent souvent Internet pour, bien entendu, communiquer, mais également pour apprendre et pour jouer à des jeux de diverses catégories. Le rapport Bourgogne, rédigé par Paré (2001), a ressorti toutes les particularités associées aux adolescents de la présente génération, toujours par rapport aux cybercommunications. En fait, la génération Internet compte toutes les personnes nées entre 1977 et 1997, ce qui fait en sorte qu'elle est échelonnée sur une période de 20 ans. Paré précise que l'une des caractéristiques distinctives de cette génération est la présence accrue des technologies de l'information et de la communication, qui font partie intégrante de la vie de la grande majorité de ces individus.

Les jeunes et jeunes adultes ayant vécu avec l'ère des nouvelles technologies n'ont pas vécu avec l'attrait de la nouveauté qu'elle a apportée; ils sont nés à l'intérieur de celleci. Les adolescents de cette génération ont plusieurs points communs, peu importe leur nationalité. Internet a fait en sorte qu'ils peuvent communiquer ensemble et connaître les cultures des uns et des autres. Il y a donc maintenant une certaine homogénéité entre les 
différentes cultures, surtout chez les plus jeunes. De plus, de nos jours, les jeunes font preuve d'une certaine impatience; ils désirent tout, et tout de suite (Paré, 2001). Il est ici possible de faire un lien avec le clavardage, puisque par le biais de ce média, ils ont accès à une réponse de l'internaute avec qui ils communiquent de façon quasi-instantanée. Ils misent sur la rapidité en coupant des parties des mots employés. Internet favorise donc cette notion d'instantanéité, étant donné que les jeunes ont accès à une réponse immédiate, autant lorsqu'ils communiquent en ligne que lorsqu'ils recherchent une information sur le réseau virtuel. Il est donc prévisible que les adolescents, qui ont pris l'habitude de l'instantanéité, généralisent celle-ci à d'autres aspects de leur vie. Effectivement, c'est ce que laisse entendre Paré en précisant que les jeunes font aujourd'hui preuve d'impatience.

Leur maîtrise de l'ordinateur étant ce qu'elle est, les adolescents ont pour la plupart développé de très bonnes habiletés en rédaction, en mathématiques et en ce qui a trait au développement de la pensée critique (Gustin, Klein et Rosenberg, 1999). Les parents approuvent et reconnaissent les bienfaits intellectuels qu'apporte Internet dans la vie de leurs enfants, notamment au niveau de leur intérêt croissant par rapport à l'actualité générale (Paré, 2001).

L'utilisation de l'ordinateur recèle en effet bien des avantages. Spécifiquement, la fréquentation de sites concernant des sujets diversifiés contribue au fait que les enfants soient plus matures, développent leurs valeurs et aient la possibilité de travailler leur jugement, ce qui peut les faire cheminer au niveau de la confiance en soi (Tapscott, 1998). Paré (2001) souligne enfin que ces adolescents ont surtout tendance à être caractérisés comme étant une génération prometteuse, des jeunes positifs, ayant un fort esprit d'équipe et respectant l'autorité lorsque la personne y ayant droit est jugée compétente.

Les statistiques montrent que les adolescents canadiens âgés entre 12 et 17 ans passent autant de temps sur l'ordinateur que devant la télévision, ce qui n'était pas le cas quelques années auparavant (Paré, 2001). Les deux médias, autant l'un que l'autre, font ainsi partie intégrante de la vie des jeunes. Il est donc facile de constater que la popularité d'Internet est en pleine effervescence. 
Il est important d'expliquer la popularité d'Internet selon les groupes correspondant à l'âge adulte. Au Québec, $63 \%$ des 18-24 ans naviguent sur Internet, alors que $54 \%$ des personnes entre 25 et 34 ans le font. Chez les 35-44 ans, ce pourcentage est de $47 \%$ (Paré, 2001). Il est ainsi facile de constater que les 18-24 ans en sont les principaux adeptes, ce qui semble correspondre aux caractéristiques de cette génération, étant donné que les individus se situant dans cette tranche d'âge font partie de la génération Internet.

Une étude du Réseau Éducation-Médias (2005) suggère pour sa part que $94 \%$ des jeunes québécois de neuf à 17 ans ont accès à Internet à la maison. En cinquième secondaire, $50 \%$ des jeunes possèdent leur ordinateur personnel. Le clavardage est l'activité la plus populaire sur Internet, cela pour $62 \%$ des filles et $63 \%$ des garçons canadiens. Les adolescents d'aujourd'hui considèrent Internet comme étant un lieu de communication et de socialisation. Également, $56 \%$ des jeunes canadiens utilisent quotidiennement le clavardage pour communiquer; $50 \%$ des jeunes québécois font appel à Internet durant au moins une heure par jour et $39 \%$ d'entre eux considèrent que l'avantage majeur d'Internet est de pouvoir communiquer avec autrui. D'ailleurs, $56 \%$ des adolescents québécois clavardent régulièrement, dont $25 \%$ quotidiennement (Réseau Éducation-Médias, 2005). En général, ceux-ci clavardent à la maison, avec leurs amis ou leurs proches. Les internautes ont la possibilité de s'adonner à d'autres activités tout en clavardant, comme écouter de la musique, faire des devoirs et rechercher des informations de tous genres, ce qui constitue un avantage non négligeable relié à cette forme de communication.

Le clavardage et les textos ont su conquérir un immense public, malgré le fait que les jeunes en sont les principaux utilisateurs. En lien avec les textos, David et Goncalves (2007) mentionnent que cinq millions de messages sont échangés en une seule journée, ce qui prouve l'ampleur de ce nouveau mode de communication, qui possède plusieurs similitudes avec le clavardage. Statistiquement, $90 \%$ des adolescents ont avoué avoir une préférence pour les textos lorsqu'ils doivent communiquer avec leurs proches, plutôt que de communiquer verbalement par le biais du téléphone. David et Goncalves précisent 
également que $78 \%$ des jeunes français, entre 18 et 24 ans, ont l'habitude d'envoyer fréquemment des textos.

En résumé, nous pouvons constater que l'actuelle génération de jeunes contribue grandement à l'immense popularité du clavardage et du monde virtuel, puisqu'ils sont particulièrement nombreux à s'adonner régulièrement au clavardage et à naviguer sur la toile.

\subsection{ASPECTS POSITIFS ET NEGATIFS DU CLAVARDAGE}

Dans le cadre de cette étude, il importe de considérer les aspects positifs et négatifs du clavardage. Concernant ses aspects positifs, celui-ci suscite notamment l'envie de communiquer par le biais d'un processus d'écriture vif. Les adolescents se sentent proches des autres internautes, puisque la communication se déroule en temps réel et qu'ils sont constamment en interaction sociale, ce qui est valorisant au niveau interpersonnel. Les jeunes développent également un esprit de collaboration. L'absence de jugement des autres internautes, étant donné qu'ils utilisent le même langage, est aussi remarquable. Par ailleurs, les adolescents utilisant ce nouveau mode de communication développent une communication fluide assez rapidement (Léger, 2006), puisqu'ils s'habituent au fil de la pratique aux particularités de la langue codée.

David et Goncalves (2007) précisent que la popularité de la langue codée peut avoir un lien avec le sentiment d'appartenance à un groupe; en effet, les internautes ont alors l'impression de faire partie d'une communauté de gens usant des mêmes procédés, ce qui les rapproche les uns des autres. Il s'agit ici de la communauté virtuelle. Tattosian (2008) ajoute qu'il s'agit également d'une question d'identité.

Anis (2000) détaille aussi les diverses composantes positives de cette récente forme de communication. Il ressort surtout le fait que le clavardage permet à la société actuelle d'élargir son champ par rapport à la communication écrite, ce qui est un aspect non 
négligeable. Il est maintenant facile de maintenir le contact avec des gens du monde entier, ce qui permet de découvrir une nouvelle culture, ainsi que ses fondements. Il est également possible de découvrir une nouvelle langue, que l'on peut qualifier de codée, qui se démarque par son originalité et ses changements graphiques, si on la compare avec la langue écrite conventionnelle.

Par ailleurs, différentes écoles ont décidé de mettre de l'avant des séances pédagogiques de clavardage. Par exemple, à l'école Fernand-Séguin de Montréal, les élèves clavardent tous les jours avec des élèves français durant une période de 45 minutes. Ils ont donc l'occasion de connaître une nouvelle culture et d'échanger avec des jeunes du même âge, mais ayant un mode de vie complètement différent, ce qui est très riche pour des enfants (Faverjon, 2003). Bien entendu, toutes leurs communications sont enregistrées et supervisées, ce qui fait en sorte qu'elles peuvent ensuite être réutilisées dans un contexte pédagogique et de correction de la langue française (Réseau de télématique scolaire québécois, 2004). De même, plus les années avancent, plus de projets pédagogiques impliquant de manière active le clavardage voient le jour, ce qui est une excellente façon de promouvoir la langue écrite tout en favorisant le plaisir et la motivation chez les jeunes.

En ce qui concerne les effets négatifs associés au clavardage, il est de circonstance de mentionner le phénomène de cyberintimidation en milieu scolaire, qui prend de plus en plus d'ampleur dans la société. Les principales personnes touchées par ce phénomène sont les élèves, puisque plusieurs d'entre eux en sont les victimes. Chantelois précise :

La cyberintimidation se déroulerait surtout par courriel (54\%), sur les sites Internet de clavardage $(20 \%)$ et par texto $(11 \%)$. Les commentaires des cyberintimidateurs portent généralement sur l'apparence des victimes. Seulement $5 \%$ des membres du personnel des établissements scolaires sont victimes de cyberintimidation. Les vraies victimes, ce sont plutôt les élèves (Chantelois, 2008).

Selon ces statistiques, $27 \%$ des élèves ayant participé au sondage connaissent au minimum une personne qui a dû faire face à un problème d'intimidation, qui peut sans aucun doute avoir une influence négative sur la vie des personnes touchées de près ou de loin par cette problématique. 
Lorsqu'ils désirent intimider des jeunes par le biais d'Internet, les jeunes le font par l'intermédiaire du clavardage et vont principalement sur des sites tels que Facebook, Myspace et MSN. Les attaques concernent principalement les sujets suivants : l'apparence physique, les propos diffamatoires, les menaces de dommages corporels, les commentaires sur l'enseignement, les menaces ou insultes à caractère sexuel, les messages haineux et les messages d'atteinte aux biens. De même :

[...] plus de $80 \%$ des Canadiens sont d'accord ou entièrement d'accord pour dire que l'intimidation est l'un des plus graves problèmes auxquels se heurtent les élèves d'aujourd'hui. Près de la moitié $(47 \%)$ des parents canadiens avouent que leurs enfants ont été victimes d'intimidation et $16 \%$ que les incidents de cette nature sont fréquents. Les Canadiens croient généralement que trop peu est fait pour prévenir l'intimidation et y mettre fin dans les écoles (Chantelois, 2008).

Parallèlement, l'institution scolaire a un rôle à jouer pour contrer les influences négatives que pourrait causer le clavardage. Les directions d'école doivent prendre des mesures pour que le clavardage ne devienne pas un problème et qu'il ne soit pas utilisé de façon problématique lors des cours consacrés à l'informatique. Selon nos expériences personnelles, lors des périodes en laboratoire informatique, les jeunes n'ont pas accès aux différents réseaux de clavardage; ceux-ci ne sont pas installés sur les ordinateurs, autant sur ceux des laboratoires informatiques que ceux en classe. Le règlement est semblable pour ce qui est des téléphones cellulaires et des textos, qui sont interdits dans certaines institutions scolaires. Sinon, il serait possible que les élèves passent beaucoup de temps sur ces outils conversationnels à l'école et finissent par mettre de côté leurs apprentissages ou même risquent de cyberintimider leurs camarades. En revanche, il est sans doute envisageable pour la plupart des écoles de prévoir des activités de clavardage pédagogique, selon le vouloir des enseignants; il suffit alors que les logiciels de clavardage soient accessibles dans ces circonstances seulement. 


\subsection{CARACTERISTIQUES PRINCIPALES DE LA LANGUE ECRITE CODEE UTILISEE LORS DE SEANCES DE CLAVARDAGE}

Dans le cadre de la présente étude, il est primordial d'étudier la langue codée utilisée par les adolescents d'aujourd'hui pour clavarder, dans le but de vérifier rigoureusement si elle est employée dans les écrits scolaires des sujets.

Les recherches menées en France par Anis (2003) se concentrent principalement sur la communication électronique et sur les nouveaux usages linguistiques francophones. Le chercheur étudie donc les caractéristiques du clavardage et des textos et les place dans une même typologie, puisqu'elles sont communes à ces deux modes de communication.

La communication écrite électronique est caractérisée comme étant rapide, volatile, immatérielle et brève. Une nouvelle variété définit le français écrit, qui est maintenant brut (sans relecture), familier, affectif (expression des sentiments favorisant le relâchement du contrôle), ludique (jeu de mots) et socialisant (partage de codes communs). D'ailleurs, divers éléments caractérisent les messages électroniques, soit : les abréviations, l'oralité, l'iconicité, les pictogrammes, les idéogrammes et les signes-syllabes. Les scripteurs sont généralement jeunes et leurs buts de communication sont fonctionnels et affectifs.

De même, les caractéristiques suivantes sont associées au clavardage: espaces collectifs et espaces privés (où les gens peuvent discuter avec plusieurs personnes à la fois ou dans des niches privées, en dyade), communication interactive et interindividuelle, communication quasi-directe (les gens discutent en ligne et ont une réponse très rapidement), messages de quelques lignes seulement, affichage éphémère (affichage à défilement rapide), corps de caractères variables, couleurs et icônes.

De plus, l'auteur s'est attardé à l'analyse des formes linguistiques fréquentes pour ce qui est du clavardage. En voici les principales : 
Tableau 1: Les formes linguistiques associées au clavardage

1. Les réductions graphiques: abréviation de lettres et de mots.

2. Les réductions avec variantes phonétiques : les scripteurs changent l'écriture des mots, malgré le fait que la prononciation reste la même.

3. L'élimination de certaines consonnes.

4. L'utilisation de lettres au lieu de mots, par exemple: c pour c'est.

5. Les étirements graphiques.

Lors des sessions de clavardage, les adolescents ont tendance à utiliser divers raccourcis de nature linguistique dans leurs écrits. Par exemple, les lettres ainsi que les mots sont substitués par des expressions phonétiques. Les lettres jugées superflues sont tout simplement enlevées. Des acronymes, qui sont souvent issus de la langue anglaise, font aussi partie des écrits des jeunes. Parfois, les gens ayant recours à ce mode de communication utilisent les lettres majuscules pour mettre l'accent sur un ou des éléments mentionnés dans une phrase (Anis, 2003).

Un langage très populaire associé au clavardage, les «smileys », que l'on peut nommer également «emoticons», est très exploité. Ces deux termes représentent des illustrations des émotions, où de petits visages sont matérialisés. Il est alors aisé de faire de petits sourires ou des clins d'œil, ce qui peut être attrayant pour le regard et amusant pour les utilisateurs. Anis précise qu'il s'agit de signes auxiliaires qui ont une influence sur la signification d'une phrase; en effet, les signes de ponctuation servent souvent de base pour illustrer les émotions, par exemple :-) sourire et ;) clin d'œil (Anis, 2000).

Par rapport à la ponctuation, Anis (2000) explique que les signes de ponctuation, mis à part pour les «smileys », sont généralement peu utilisés lors d'une communication par le 
biais du clavardage; les gens ne prennent souvent pas la peine de mettre une majuscule au début de leur phrase et un point à la fin de celle-ci. De plus, la ponctuation de nature expressive et interrogative ( !, ?) est utilisée de façon hors-norme, c'est-à-dire que les utilisateurs vont inscrire plusieurs points d'exclamation ou d'interrogation à la fin de leur phrase, dans le but de mettre plus d'emphase sur leur discours. Il s'agit d'une pratique courante sur les sites de clavardage. Ces éléments attirent également le regard et retiennent l'attention de l'internaute avec qui ils sont en communication.

Anis (2000) aborde en détail les caractéristiques de la langue codée développée dans le cadre de l'utilisation du clavardage. D'une part, les gens ont tendance à réduire le nombre de mots qu'ils utiliseraient normalement lors de l'écriture d'une phrase; cela est plus rapide et constitue l'un des buts de la cybercommunication. D'autre part, plusieurs utilisateurs vont chercher à s'exprimer par le biais de cette langue codée, ce qui fait en sorte que certains d'entre eux vont transgresser la norme de façon à se faire remarquer davantage. Par exemple, les lettres $q u$ vont régulièrement être modifiées pour la lettre $k$, qui se prononce de la même façon; les gens peuvent donc écrire plus rapidement, puisqu'ils ont une lettre de moins à taper. Il s'agit presque d'une norme dans la communication électronique. Donc, au lieu d'écrire «Qui vient au cinéma ce soir? », le scripteur écrira : « Ki vient au cinéma ce soir? ». Les clavardeurs ont aussi tendance à écrire une seule lettre au lieu d'un mot lorsque celle-ci se prononce de la même manière. La lettre $c$ peut alors souvent remplacer les mots $c$ 'est, puisque sa prononciation est semblable. Il s'agit de la même chose pour la lettre $t$, cette fois-ci au lieu des mots tu es. Également, les lettres qui ne sont pas jugées nécessaires pour la compréhension du mot sont tout simplement enlevées (beaucoup s'écrira $b c p$ ).

De plus, des expressions sont utilisées dans l'objectif de remplacer certains groupes de mots. Par exemple, $m d r$ signifie mort de rire; les internautes désirent donc spécifier qu'ils trouvent une situation amusante. Lorsque ceux-ci demandent asv à la personne avec qui ils discutent, ils désirent connaître l'âge, le sexe et la ville de cet individu. Les habitués du clavardage connaissent cette panoplie d'expressions que les gens inconnus à ce milieu 
ne sauraient reconnaitre. De même, les étirements graphiques sont également couramment employés, ce qui velit dire que les usagers vont ajouter des lettres à la fin de certains mots

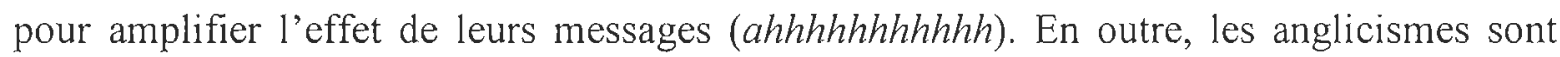
aussi très présents, particulièrement chez les Québécois et les Français, qui utilisent déjà plusieurs anglicismes dans leur discours oral. Comme le précise Anis, il suffit de penser aux mots «cute» et «chum» pour reconnaître que ces mots sont particulièrement populaires dans notre langage de tous les jours (Anis, 2000).

Les jeunes communiquant entre eux par l'intermédiaire du clavardage ont donc l'habitude d'abréger, de styliser et de transformer les mots pour accélérer ou simplifier la conversation. Anis a comparé un texto envoyé par une élève et un mot écrit rédigé par cette même élève, qui était destiné à une camarade de classe. 11 a remarqué des expressions semblables et s'est posé la question suivante: "Cet écrit scolaire ou anti-scolaire peut-il détourner les jeunes de l'apprentissage ingrat d'une norme orthographique complexe comme celle du français $»$ ? (Anis, 2003).

En France, David et Goncalves (2007) ont travaillé sur les caractéristiques de la langue codée utilisée dans les textos. Tel que nous l'avons vu précédemment dans les écrits de Anis, cette langue codée est commune au clavardage et aux textos. En ce qui les concerne, ils précisent que lors de l'envoi d'un message de ce type, l'usager doit favoriser certains éléments. Tout d'abord, le message doit être court et sa transmission doit user de peu de lettres ou de signes. Souvent, cette contrainte est due au volume auquel a droit le propriétaire du téléphone cellulaire. Ces réductions font en sorte que les jeunes d'aujourd'hui utilisent davantage de symboles, de pictogrammes et d'abréviations. Certes, les mots sont souvent écrits comme ils sont prononcés à l'oral, ce qui permet de faire le lien entre la langue codée et l'oralité, qui sera présenté au point 2.7. David et Goncalves caractérisent cette langue codée comme étant une «invention scripturale » et ces derniers semblent avoir raison de le faire, puisque cette écriture est réellement une invention causée par le manque d'espace, le temps déficient et le caractère immédiat des réponses attendues. 
David et Goncalves rapportent également que les élèves ayant de grandes difficultés en français ne prennent pas le temps de simplifier ou d'inventer de nouvelles écritures, puisqu'ils ne maitrisent pas encore les fondements de l'écriture conventionnelle. Effectivement, s'ils désirent faire des modifications graphiques, ils doivent d'abord et avant toute chose avoir accès à une orthographe convenable.

Enfin, David et Goncalves (2007) ont établi une typologie détaillée de la langue codée utilisée par l'intermédiaire des textos, dont les caractéristiques sont semblables à celles attribuées au clavardage.

\subsubsection{Les réductions graphiques}

Les réductions graphiques constituent en une coupure du nombre de lettres dans un mot, ce qui fait en sorte que l'écrit se rapproche de l'oral, puisque le mot s'écrit alors comme il se prononce. Voici quelques exemples: 
Tableau 2: Les réductions graphiques

\begin{tabular}{|c|c|}
\hline $\begin{array}{l}\text { Mots ou expressions dont le } \\
\text { style graphique est modifié }\end{array}$ & $\begin{array}{l}\text { Changements graphiques } \\
\text { occasionnés et exemples }\end{array}$ \\
\hline $\begin{array}{l}\text { 1) diminution du } \\
\text { phonogramme } q u\end{array}$ & $\begin{array}{l}\text { 1) qu devient k (koi au lieu de } \\
\text { quoi) }\end{array}$ \\
\hline 2) modification de la lettre $c$ & $\begin{array}{l}\text { 2) c devient } \mathrm{k} \text { (kom au lieu de } \\
\text { comme) }\end{array}$ \\
\hline 3) modification de la lettre $s$ & $\begin{array}{l}\text { 3) s devient z (biz au lieu de } \\
\text { bises) }\end{array}$ \\
\hline $\begin{array}{l}\text { 4) enlèvement des e qui ne } \\
\text { se prononcent pas }\end{array}$ & 4) styl (au lieu de style) \\
\hline $\begin{array}{l}\text { 5) enlèvement de lettres } \\
\text { quelconques qui ne se } \\
\text { prononcent pas }\end{array}$ & 5) je sui (au lieu de je suis) \\
\hline $\begin{array}{l}\text { 6) modification complète } \\
\text { des phonogrammes, ce } \\
\text { qui va en contre-sens de } \\
\text { l'économie de temps. }\end{array}$ & 6) toua (toi) \\
\hline $\begin{array}{l}\text { 7) diminution des groupes } \\
\text { de mots }\end{array}$ & 7) jle sé (je le sais) \\
\hline
\end{tabular}

\subsubsection{Les réductions et transformations avec variantes phonétiques}

David et Goncalves caractérisent les réductions et transformations avec variantes phonétiques comme étant des «déformations de la langue standard, dans sa forme écrite mais aussi orale » (David et Goncaives, 2007: 4). Ils précisent que la majorité des utilisateurs se servent de plusieurs modifications de mots à la fois; ils ne s'en tiennent donc pas à une seule catégorie. Il peut arriver en conséquence que le message soit difficile à comprendre et manque de clarté. Il demeure que les internautes doivent respecter les 
normes de ce nouveau langage pour, en quelque sorte, être intégrés dans la communauté virtuelle.

Tableau 3: Les réductions et transformations avec variantes phonétiques

\begin{tabular}{|c|c|}
\hline $\begin{array}{l}\text { Mots ou expressions dont le } \\
\text { style graphique est modifié }\end{array}$ & $\begin{array}{l}\text { Changements graphiques } \\
\text { occasionnés et exemples }\end{array}$ \\
\hline 1) variantes vocaliques & 1) toa (toi) \\
\hline $\begin{array}{l}\text { 2) écrasement du mot pour } \\
\text { qu'il corresponde au son }\end{array}$ & 2) koi (quoi) \\
\hline $\begin{array}{l}\text { 3) écriture des consonnes } \\
\text { principales pour écrire un } \\
\text { mot }\end{array}$ & 3) ds (dans) \\
\hline $\begin{array}{l}\text { 4) écriture d'une lettre } \\
\text { seulement représentant un } \\
\text { mot }\end{array}$ & 4) d (des); j (je) \\
\hline $\begin{array}{l}\text { 5) acronymes représentant une } \\
\text { expression courante en } \\
\text { informatique }\end{array}$ & 5) $\mathrm{mdr}$ (mort de rire) \\
\hline $\begin{array}{l}\text { 6) étirements graphiques, pour } \\
\text { mettre l'emphase sur le } \\
\text { message }\end{array}$ & 6) je suis laaaaaaaaaaa! \\
\hline
\end{tabular}

\subsubsection{Particularités de ce mode de communication}

Pour ces deux chercheurs, la combinaison de ces différentes stratégies d'écriture met en lumière un nouveau lexique. En effet, les procédés d'écriture sont diversifiés, originaux et créatifs, sans pour autant être incompréhensibles (David et Goncalves, 2007). Voici maintenant les particularités générales de ce mode de communication. 
Tableau 4: Particularités de ce mode de communication

\begin{tabular}{|c|c|}
\hline Caractéristiques & Explications \\
\hline 1) hétérogénéité & $\begin{array}{l}\text { 1) un seul mot peut combiner } \\
\text { plusieurs procédés. }\end{array}$ \\
\hline 2) polyvalence & $\begin{array}{l}\text { 2) un même mot peut être écrit } \\
\text { de différentes manières. }\end{array}$ \\
\hline 3) variation & $\begin{array}{l}\text { 3) la même unité lexicale est } \\
\text { écrite de plusieurs façons. }\end{array}$ \\
\hline 4) verlan & $\begin{array}{l}\text { 4) un langage oral populaire, } \\
\text { le « verlan », est employé, } \\
\text { surtout en France. }\end{array}$ \\
\hline 5) troncations & $\begin{array}{l}\text { 5) les troncations consistent en } \\
\text { des abréviations de mots, en } \\
\text { coupant leurs dernières } \\
\text { lettres. }\end{array}$ \\
\hline 6) onomatopées & $\begin{array}{l}\text { 6) elles sont couramment } \\
\text { utilisées, particulièrement } \\
\text { combinées à des étirements } \\
\text { graphiques. }\end{array}$ \\
\hline
\end{tabular}

Il y a tout de même des éléments du français conventionnel que nous retrouvons dans le français informatisé, malgré le fait que ses règles d'usage semblent disparaître de plus en plus. Notamment, nous pouvons observer que les mots sont séparés de façon convenable, c'est-à-dire que l'utilisateur prend toujours le temps de mettre les espaces nécessaires entre les mots. Également, plusieurs mots demeurent écrits de la bonne façon, même si ce n'est pas le cas pour tous les internautes; ce ne sont donc pas tous les mots qui subissent une mutation. Comme le mettent en évidence David et Goncalves (2007), il est impossible de changer tous les mots de la langue française, puisque le message serait alors incompris. 
Au Québec, Tatossian (2008) s'est attardée aux échanges entre adolescents et entre adultes dans les forums de clavardage, où la rapidité et la simultanéité sont de mise. Il en est ressorti que les adultes transgressent presque autant la langue française que les jeunes, ce qui fait en sorte que la faiblesse des plus jeunes envers la langue n'est pas nécessairement en cause. Par contre, les adolescents ont plus tendance à inventer un code où leur identité peut se refléter. Par exemple, ils vont davantage utiliser de petits icônes où ils peuvent faire connaître leurs émotions (sourires, clins d'œil, grimaces...) à leurs correspondants.

Les études sur le clavardage s'entendent sur l'importance de deux facteurs: le temps, qui incite les clavardeurs à recourir à des stratégies d'abréviation et l'emploi de marques d'expressivité qui ont pour effet de réduire l'écart entre l'oral et l'écrit. Étant donné que le clavardage consiste à établir une communication en temps réel fondée uniquement sur le texte, sans transmettre l'image ou la voix des scripteurs, les clavardeurs sont tentés de reproduire les paramètres de la conversation en face-à-face : expressions faciles, intonations, pauses, expressions des émotions (Tattosian, $2008: 1$ ).

Par ailleurs, Paré (2001) mentionne que les discussions se réalisant par l'intermédiaire du clavardage, bien qu'elles soient écrites, ne permettent pas aux internautes de cerner les indices permettant de savoir dans quel état d'esprit se trouve l'autre individu. La discussion est donc moins personnelle. Par exemple, lorsqu'une personne discute face à face avec quelqu'un, il est possible de distinguer quelles émotions la traversent en prêtant attention à son ton de voix et en examinant l'expression de son visage. Il écrit à ce sujet :

C'est ainsi qu'est née une nouvelle écriture: nouvelles combinaisons de caractères, nouvelles abréviations, nouveaux acronymes, création de néologismes ayant pour but d'intégrer à la communication écrite certaines des subtilités et des émotions présentes dans l'échange direct (Paré, 2001: 17).

De l'avis de Robin (2000), les environnements virtuels ayant comme base la communication entre internautes changent complètement l'expérience du langage. En fait, le côté interactif de cet outil de communication amène cette transformation. Des formes langagières innovatrices ont fait leur apparition, de même qu'une différente textualité, étant donné que ces discussions écrites se déroulent devant un écran. En outre, à l'instar 
d'intituler ce langage comme étant une langue codée, Robin la caractérise comme étant une langue hybride, une langue d'usage modifiée de diverses façons et ayant plusieurs ressemblances avec un langage de programmation informatique (Robin, 2000).

Jaffré (2006) caractérise la langue utilisée lors de conversations électroniques comme étant de « nouvelles écritures ». Il mentionne que l'écriture doit être phonographique, ce qui fait en sorte qu'elle doit posséder un codage minimal, combiné de syllabes et de phonèmes. De plus, le deuxième principe de l'écriture concerne la sémiographie, où des signes sont représentés par son intermédiaire, autant des morphèmes que des mots. Ces deux principes font en sorte qu'une certaine polyvalence au niveau de l'orthographe est au centre de l'écriture.

L'auteur explicite que l'orthographe utilisée en situation de clavardage est bien différente de celle enseignée à l'école. Il caractérise cette nouvelle écriture comme étant «le reflet des nécessités minimales auxquelles une écriture doit satisfaire pour remplir une fonction de communication » (Jaffré, 2006), ce qui définit très bien le caractère minimal de cette écriture codée.

Jaffré a pour opinion que l'écriture utilisée dans le cadre de conversations par textos se rapproche de l'écriture des jeunes enfants qui débutent tout juste à écrire et qui ne connaissent pas encore les normes de l'écriture. Lorsqu'ils débutent à écrire, les jeunes enfants vont puiser dans leurs connaissances pour réfléchir à la façon dont les mots s'écrivent, en se référant à des indices grapho-phonétiques qu'ils ont gardés à l'esprit. Contrairement aux enfants, les adultes vont choisir la façon dont ils désirent écrire les mots, ce qui n'empêche en rien que plusieurs d'entre eux utilisent le même code que les plus jeunes lorsqu'ils communiquent par le biais du clavardage (Jaffré, 2006).

Enfin, Panckhurst (1999) a étudié les caractéristiques de nature linguistique caractérisant le courrier électronique, de même que le discours étant utilisé lors de son utilisation. Étant donné les similitudes entre le courrier électronique et le clavardage, il est pertinent, dans le contexte actuel, de s'intéresser à ses constatations. 
Les sujets participant à cette recherche sont des étudiants universitaires de premier cycle divisés en deux groupes. Le premier groupe comportait 115 messages envoyés par courrier électronique, rédigés par 64 étudiants d'un cours axé sur l'informatique. Le second groupe, quant à lui, était constitué de 1285 messages, écrits par des collègues, des amis et des étudiants aux cycles supérieurs. Ces écrits totalisent 1676 messages. L'étude s'est déroulée sur une période de huit mois. Un traitement d'analyse morpho-syntaxique a ensuite été réalisé.

Entre autres, Panckhurst a remarqué que les erreurs dites de base (erreurs de frappe ou erreurs lexico-grammaticales) sont assez communes dans le courrier électronique, étant donné que les gens ne prennent souvent pas le temps de se relire.

Panckhurst explique ces erreurs comme suit :

Par contre, et cela pourrait surprendre davantage, y compris dans les messages provenant de collègues universitaires, les erreurs sont toutes aussi fréquentes. Pour une part, cela s'explique aisément : le courrier électronique est un moyen rapide de communication; de ce fait, on se relit peu, et les erreurs (qui peuvent toujours être rejetées sur l'outil informatique) sont, de manière générale, relativement bien tolérées (Panckhurst, $1999: 3$ ).

En résumé, nous pouvons constater que le clavardage a amené plusieurs modifications langagières par rapport au français conventionnel, ce qui fait en sorte que ce mode de communication virtuel possède une langue codée qui lui est propre, avec les textos, bien entendu. Principalement, en situation de clavardage, les mots et les expressions francophones subissent des réductions graphiques et des réductions et transformations avec variantes phonétiques. Bien sûr, plusieurs particularités caractérisent ce langage, dont son hétérogénéité, son caractère variable et sa polyvalence. 


\subsection{Clavardage et oralite}

Il est intéressant de comparer plus en détail le clavardage à l'interaction orale puisqu'ils possèdent plusieurs ressemblances. En effet, les chercheurs s'intéressant à la manière dont s'acquièrent les langues et à la communication par le biais de l'informatique ont remarqué des ressemblances flagrantes entre les discussions en situation de clavardage qui se réalisent par l'intermédiaire de l'ordinateur et les discussions orales se déroulant d'une personne à l'autre, face à face (Tudini, 2003).

Kern (1995) et Warschauer (1996) ont comparé lors de deux études distinctes la langue écrite utilisée par le biais du clavardage et la langue orale. Kern (1995) a réalisé une analyse d'archives par rapport au clavardage et à la langue française. Il a constaté que les phrases sont moins travaillées et moins longues lorsque deux personnes clavardent que lorsqu'elles discutent verbalement. Par contre, l'étude réalisée par Warschauer (1996) vient contredire ce fait. Ce chercheur, qui s'est quant à lui attardé à la langue anglaise seconde, précise que les clavardeurs utilisent une langue plus complexe aux niveaux lexical et syntaxique que lors d'échanges oraux en face à face.

Voici quelques caractéristiques conversationnelles souvent observées lors des sessions de clavardage, qui s'apparentent à celles de l'interaction orale. Elles ont été constatées par Negretti (1999), qui a réalisé une recherche lors de laquelle elle a observé des séances de clavardage. Les voici :

Les aspects essentiels de l'interaction orale analysés par Negretti lors de sessions de clavardage sont: la structure globale de l'interaction et l'organisation des séquences, l'organisation interne des interventions, l'architecture des tours de parole, l'expression des aspects paralinguistiques et quelques autres variables pragmatiques. Ces caractéristiques conversationnelles sont toutes présentes dans les sessions de clavardage [...] (Tudini, $2003: 64$ ). 
Par rapport à la capacité d'expression orale des participants à l'étude, celle-ci s'est améliorée deux mois après les débuts de cette dernière, lors desquels plusieurs activités de clavardage ont eu lieu (Negretti, 1999).

Bernier (2007), en ce qui la concerne, caractérise le produit des discussions par l'intermédiaire du clavardage comme étant des « écrits conversationnels » étant donné que ces discussions se rapprochent beaucoup des discussions orales. Effectivement, les utilisateurs de ce mode de communication ont tendance à écrire les mots tels qu'ils les prononcent à l'oral.

Par ailleurs, comme le précisent Labbé et Marcoccia (2005), «le dialogue par courrier électronique serait, selon ces hypothèses, une pratique de communication déterminée par les normes et les conventions de l'échange oral et-ou de la lettre ». Ils mettent en évidence eux aussi que, lors d'une session de clavardage, la langue utilisée est écrite, mais possède plusieurs points communs avec une interaction orale étant donné que les échanges se déroulent selon une structure de dialogue; le caractère rapide de l'écriture des messages de même que leurs transmissions sont relatifs au clavardage, mais également à l'interaction orale.

Filatova (2006) remarque que l'avènement du clavardage est tout un défi pour l'intelligence humaine. Effectivement, elle met en évidence que le clavardage se définit notamment par l'absence d'éléments majeurs qui caractérisent la conversation. Notamment, la parole n'est plus obligatoirement de niveau sonore. De plus, l'internaute n'a pas à être présent physiquement pour avoir une conversation avec un autre individu.

D'autre part, tel que le souligne cet auteur, la personne n'a aucune idée de l'état d'esprit de l'individu avec qui elle discute ainsi que de l'ambiance du lieu dans lequel il se trouve lorsqu'elle clavarde. Elle ne peut donc pas s'attarder aux subtilités de la conversation. En somme, selon Filatova : 
[...] nous avons entre les mains un texte qui, dans la situation de l'absence flagrante de trois composantes indispensables de la communication, se fait passer pour une conversation, et tout le monde cède à cette illusion assez bizarre tout en étant parfaitement à l'aise (Filatova, $2006: 138$ ).

Quant à Luzzati (1991), il a constaté que plusieurs écrits particuliers ont des liens étroits avec l'oral étant donné que parfois les conditions de production sont davantage semblables à l'oral qu'à l'écrit. Le chercheur souligne par rapport à l'oralité dans les écrits virtuels que l'oral n'est pas créé pour être lu, malgré le fait que le discours des écrits virtuels consiste selon lui en de l'oral. En fait, ces écrits ne doivent pas être lus de manière générale puisque cela n'en est pas le but. Ils doivent avoir un objectif de communication ciblé.

En résumé, nous pouvons constater que les échanges réalisés par l'intermédiaire du clavardage possèdent plusieurs similitudes avec les échanges oraux. En fait, certains sont d'avis que les discussions par le biais du clavardage se rapprochent davantage de l'oral que de l'écrit. Les propriétés de ce média sont donc souvent associées à celles utilisées lors d'échanges se déroulant oralement, en face à face ou en groupe.

\subsection{OPINIONS SUR L'EFFET DU CLAVARDAGE SUR LA MAITRISE DE LA LANGUE FRANCAISE ECRITE}

Ainsi, tel que vu précédemment, le clavardage est caractérisé par une langue spécifique qui a pour objectif de rendre le temps de réponse plus rapide. Souvent, les personnes communiquant par le biais de ce média ne réfléchissent pas à l'orthographe, à la syntaxe et aux autres éléments associés au français écrit. Ils désirent simplement se faire comprendre. Les fautes n'ont donc pas d'importance, du moment que le message est bel et bien compris. Les adolescents utilisant le clavardage exploitent eux aussi cette langue que l'on pourrait qualifier de codée. Par contre, en ce qui les concerne, leur apprentissage du français n'est pas encore achevé. Recourir à cette nouvelle langue, qui ne correspond pas aux normes de la langue française, peut-il nuire à la maîtrise de leur français écrit? Feront- 
ils la distinction entre la langue codée et le français conventionnel lorsqu'ils seront en situation d'écriture?

Les opinions exprimées dans les médias quant à l'influence du clavardage sur la maîtrise du français écrit divergent. Dans un article publié dans Le Soleil, Dion-Viens (2008) expose le point de vue de Ouellon, selon lequel le français ne doit pas craindre la langue codée utilisée en contexte de clavardage par les adolescents d'aujourd'hui. En sa qualité de président du Conseil supérieur de la langue française, il affirme que cette nouvelle façon de s'exprimer au clavier met en évidence la capacité d'adaptation des jeunes quant à la maîtrise de la langue. Il minimise également l'importance des fautes d'orthographe. «Il ne faut pas faire un drame avec ça (le langage texto), comme il ne faut pas en faire un avec la faute. Qu'est-ce qui est le plus important, savoir bien structurer un texte ou ne pas faire de fautes? Je préfère quelqu'un qui sait comment organiser sa réflexion » (Dion-Viens, 2008).

D'ailleurs, Ouellon (2010) a prononcé une allocution lors de la Rencontre des gestionnaires de l'éducation concernant la langue d'enseignement. Il a notamment fait une comparaison entre le discours des élèves d'aujourd'hui de ceux ayant appris le français des années auparavant. Selon lui, il faut dédramatiser le contexte actuel en gardant à l'esprit que les individus ayant été éduqués lors des années antérieures ont tendance à idéaliser les apprentissages passés correspondant à leur époque. Plusieurs pays vivent en quelque sorte une crise des langues puisque la qualité de celles-ci tend à se détériorer avec les années. Effectivement, il souligne que non seulement le Québec est pris dans cet engrenage, mais également la Suisse, la Belgique et les États-Unis.

Ouellon explique qu'il n'est pas nouveau de dire que le niveau de l'écriture a baissé considérablement. En effet, des affirmations de ce genre étaient entendues même avant l'avènement du clavardage. Il précise que la langue utilisée lors de séances de clavardage n'aide pas à la maîtrise de la langue, mais que les jeunes sont conscients qu'il ne s'agit pas du niveau d'écriture qu'ils doivent employer en classe. Léger (2006), quant à elle, mentionne qu'il faut toujours que les jeunes gardent à l'esprit qu'il s'agit d'un code qui doit 
s'exprimer seulement dans un contexte particulier; ils doivent être aptes à comprendre qu'il ne s'agit pas d'une langue de tous les jours.

En revanche, il demeure que Ouellon (2010) met quand même la faute des modifications langagières survenues au cours des dernières années sur l'ère des changements technologiques, de la démocratisation des stratégies d'enseignement et de la facilité avec laquelle nous pouvons trouver des renseignements provenant du monde entier sur Internet lorsque nécessaire; tous ces éléments ont d'après lui contribué au fait que le français a subi des changements majeurs lors des dernières années. Toutefois, il ne remet pas en question le clavardage et le voit toujours d'un bon côté. Il précise que les jeunes utilisent adéquatement les technologies de l'information et de la communication, alors que ce n'est pas le cas pour plusieurs de leurs parents. Ceux-ci maitrisent donc un média que leurs parents connaissent à peine, ce qui est nouveau pour le monde éducatif. Ouellon caractérise l'univers de l'informatique comme étant «un mode de pensée qui échappe aux adultes », ce qui est pertinent par rapport à ce que l'on sait des aptitudes et de l'intérêt des plus jeunes envers cette nouvelle technologie.

Les jeunes d'aujourd'hui écrivent davantage. Par contre, ils ne le font pas nécessairement de manière à améliorer leurs performances scolaires. Avant, il fallait avoir un but précis pour écrire. Aujourd'hui, de plus en plus de jeunes écrivent par le biais du clavardage et de la téléphonie cellulaire sans avoir un objectif de communication ciblé. D'ailleurs, comme il l'a été précisé précédemment, les jeunes écrivent souvent comme ils entendent, ce qui s'apparente à l'oral (Ouellon, 2010). Bref, selon Ouellon, il demeure que le niveau d'écriture a baissé lors des dernières années. Toutefois, selon lui, le clavardage n'est pas en cause.

Selon l'avis de Nolet (2006), qui agit comme conseiller pédagogique, il n'est pas nécessaire de défier le caractère attractif du clavardage. Effectivement, l'immense popularité de ce média fait en sorte qu'il serait difficile pour les gens qui en ont développé une habitude de s'en priver. Au contraire, les acteurs de l'éducation doivent faire preuve 
d'adaptation en demandant aux jeunes de prêter davantage attention aux règles de grammaire et aux règles générales de la langue française.

Du côté de la linguistique, Fairon, dont le point de vue a été rapporté par Doyon (2007), précise que les jeunes d'aujourd'hui sont multilingues, soit qu'ils sont aptes à comprendre plusieurs langues écrites, telle que la langue codée et la langue conventionnelle, et qu'ils mélangent rigueur et liberté lors de leurs séances de clavardage. Selon lui, les jeunes seraient donc capables de différencier les deux codes d'écriture selon le contexte. Par ailleurs, Nadeau, interrogée aussi par Doyon (2007), mentionne que l'utilisation combinée du clavardage et des textos peut davantage nuire aux jeunes du primaire qui ne maitrisent pas encore la langue écrite. Elle explique qu'un nouveau code est en train de naître et qu'il pourrait éventuellement passer à l'usage. Également, Côté, lors d'une entrevue avec La Haye (2006), a mentionné qu'il est d'avis que le langue utilisée lors de séances de clavardage, qui fait souvent appel à des mots anglais, n'est pas une menace pour la langue française. De plus, Tatossian (2007), aussi interrogée par Doyon, a étudié la langue associée au clavardage dans le cadre de ses études doctorales et est elle aussi d'avis que le clavardage n'est pas nuisible pour la qualité de la langue.

Une enseignante de français de troisième et de quatrième secondaire souligne que les élèves d'aujourd'hui sont capables de distinguer ce qu'ils doivent écrire lorsqu'ils clavardent et lorsqu'ils rédigent un texte qui sera évalué en contexte scolaire. Selon celle-ci, ils sont capables de garder à l'esprit qu'il s'agit de deux façons d'écrire qui sont bien différentes. Par contre, elle explique qu'il peut y avoir quelques mots dans les productions écrites des jeunes qui semblent être issus du clavardage, surtout lorsque l'élève fait un travail rapidement, sans faire attention à ce qu'il écrit (Dion-Viens 2006).

Sarrasin (2010) a interrogé Laberge et Roux. Ceux-ci sont d'avis que le clavardage change les normes de l'écriture que nous utilisons habituellement et remue les règles constituant sa base. Laberge et Roux se sont questionnés par rapport aux conséquences de cette récente technologie. Ils songent qu'il y aura lieu de s'inquiéter seulement si ce style d'écriture se transpose dans les écrits courants. Souvent, les utilisateurs ne se posent pas de 
questions lorsqu'ils clavardent; toutefois, il faut espérer qu'il en est autrement dans les écrits rédigés dans d'autres contextes, particulièrement en contextes scolaire et professionnel.

Cerisier (2003), en ce qui le concerne, aborde le pessimisme mis en évidence dans plusieurs médias par rapport aux compétences langagières des jeunes d'aujourd'hui, autant au niveau de la rédaction qu'au niveau de la compréhension de textes. Par contre, il avoue que cette faiblesse de la langue reste selon lui étonnante étant donné que les jeunes écrivent de plus en plus, surtout lorsqu'ils discutent avec leurs pairs par le biais d'un média électronique. Assurément, les jeunes composent énormément, mais pas nécessairement d'une façon qui favorise l'amélioration de leur français. Lors de communications par le biais du clavardage ou par texto, les fautes grammaticales, syntaxiques, lexicales et stylistiques sont très faciles à remarquer puisqu'elles sont courantes. De plus, les fautifs ne sont pas seulement les jeunes puisque les adultes en font autant qu'eux. Selon l'auteur, la nécessité de recourir à des raccourcis, pour sauver du temps et de l'argent (pour les textos), est l'une des causes principales de ces erreurs. D'ailleurs, selon Bourny, Braxmeyer, Dupé, Remond, Robin et Rocher (2002), une régression des performances en français à la fin du collège a été constatée et la faute est souvent rejetée sur la télévision, Internet et la téléphonie mobile; elle est par conséquent renvoyée sur les nouvelles technologies de l'information et de la communication.

Watrelot (2008) a synthétisé les principaux éléments discutés lors du colloque international «De la France au Québec : l'écriture dans tous ses états ». Elle explique que le niveau de maîtrise de l'écrit est tout simplement insuffisant, cela selon différents aspects, principalement en ce qui concerne l'orthographe générale et la qualité de l'expression écrite. Toutefois, la chercheuse ne rejette pas la faute sur l'écriture électronique. Elle indique aussi que cette faible maîtrise de la langue se constate non seulement dans les écoles secondaires québécoises et françaises, mais également dans les universités, ce qui inquiète davantage. Elle met en évidence que les enseignants se sentent peu outillés face à cette lacune, ce qui fait en sorte qu'il est difficile pour eux d'aider les jeunes à apprécier et 
à comprendre les caractéristiques de l'écriture. Bien sûr, certains éléments favorisent le déroulement d'une production écrite, dont les capacités à bien écrire. Ces capacités sont particulièrement dues aux habiletés de l'élève, au caractère efficace des méthodes d'enseignement en classe et au contexte lors duquel l'épreuve d'écriture se déroule. Waterlot précise également que l'ère d'Internet a permis à la communication écrite de se développer davantage, que ce soit au niveau personnel, professionnel ou social.

La question suivante a été posée à Meney, lors d'une entrevue menée par RadioCanada (2007) : Faut-il s'inquiéter de ce nouveau code ? En tant que linguiste, celui-ci juge qu'il faut s'en inquiéter et ne pas le faire à la fois. Il est certain qu'il s'agit d'un code tout à fait nouveau, mais il précise aussi qu'il caractérise toute une génération. Voici son point de vue :

$\mathrm{Au}$ début, dit-il, il avait l'impression que les gens faisaient la distinction entre ce nouveau code et la langue traditionnelle et son écriture enseignée à l'école. " Je suis en train de me poser la question pour savoir si c'est vraiment le cas, finalement. » Il note que les adultes qui écrivent sur les forums rejoignent les ados dans ce domaine. Même si, comme le souligne $M$. Meney, c'est une manière moins codée. "Si on lit les forums, on voit que l'orthographe en prend pour son rhume. Il y a énormément de fautes de syntaxe. Ça handicape beaucoup la lecture. On a un problème, il faut réagir (Radio-Canada, 2007).

Chouinard (2002) a interrogé Sylvain Simard, qui a déjà agi comme ministre de l'éducation. En sa qualité d'ancien enseignant de littérature, celui-ci mentionne qu'il voit avec une certaine inquiétude l'essor du clavardage. Effectivement, il précise que cette forme de communication comporte des dangers. Voici ce qu'il mentionne: «Il va falloir sensibiliser parents et enfants au fait que ces communications, qui en principe seraient en mesure d'encourager l'écriture, pourraient être reproduites un peu partout par les jeunes. » Ces paroles démontrent donc que cet ancien homme politique voit le clavardage d'un œil craintif. Il laisse clairement entendre qu'il faut faire de la prévention à cet égard.

En somme, les opinions concernant l'effet du clavardage sur la maîtrise du français écrit sont mitigées. Effectivement, certaines personnes sont d'avis que le français ne doit pas craindre l'avènement du clavardage et en voient principalement des avantages, tandis 
que d'autres sont d'un avis contraire, c'est-à-dire qu'elles considèrent qu'il est nécessaire de prévenir la population quant à une possible influence néfaste du clavardage sur la maitrise de la langue française écrite. D'autres ont des opinions nuancées ou semblent hésiter entre ces deux positions. Les quelques recherches sur l'incidence du clavardage sur la langue permettent de se faire une meilleure idée du phénomène à l'étude.

\section{9 ÉTUdes SUR L'INFLUENCE dU CLAVARDAGE SUR LA LANGUe}

Une étude anglophone (Varnhagen et al., 2009) visant à déterminer si le clavardage a une influence sur la langue a été réalisée dernièrement. Cette étude avait pour objectif d'explorer la nature des mots produits par le clavardage, ainsi que leurs fonctions, et d'examiner les relations entre l'habileté d'épeler et l'utilisation de cette nouvelle langue. Varnhagen et son équipe ont fait passer des tests d'épellation à 40 élèves qui clavardent âgés de 12 à 17 ans, tests basés sur les mots utilisés lors de séances de clavardage. L'étude démontre que les jeunes anglophones peuvent épeler correctement les mots qu'ils utilisent lorsqu'ils clavardent, ce qui suggère que celui-ci n'a pas d'effet négatif sur la langue conventionnelle anglaise. Dans l'étude en question, il est même précisé que l'utilisation du clavardage et des textos pourrait aider au développement des compétences langagières des adolescents, étant donné que les jeunes qui s'amusent avec le langage finissent par développer des aptitudes langagières. De plus, bien que cela ne soit pas significatif, les garçons ont obtenu de moins bons résultats que les filles aux tests d'épellation. Certains éléments associés à cette étude se doivent toutefois d'être soulevés. D'une part, l'échantillon est assez faible, puisque 40 jeunes seulement ont participé à l'étude. D'autre part, les analyses sont basées sur l'épellation des mots utilisés par le biais du clavardage, ce qui ne demande pas la même habileté que d'écrire des mots. Les sujets peuvent penser à la façon dont un mot s'épelle, mais qu'en est-il lorsqu'ils doivent l'écrire dans un contexte d'évaluation ou de rédaction? De plus, il reste à vérifier si des conclusions similaires s'appliqueraient pour la langue française. 
Toujours au niveau anglophone, Doyon (2007) a interrogé Tagliamonte, qui a fait une synthèse des changements linguistiques observés lors de séances de clavardage effectuées par 71 adolescents âgés entre 16 et 19 ans. Il en est ressorti que ces adolescents utilisent en contexte de clavardage de nombreuses abréviations, mais que celles-ci représentent seulement $2,4 \%$ de l'ensemble des mots du vocabulaire conventionnel anglophone. En effet, les jeunes font davantage appel à des mots courants. La chercheuse précise également que l'utilisation des différentes langues (anglais conventionnel, langue codée, langue parlée...) démontre que les adolescents maîtrisent bien la langue anglaise (Doyon, 2007).

Il est de circonstance, par rapport au sujet de la présente recherche, de se questionner sur les différences inter-langues. En effet, quelques études anglophones ont été réalisées sur le clavardage et des questions ont été soulevées étant donné les différences majeures entre le français et l'anglais, particulièrement au niveau de leur difficulté et de leur syntaxe. Il y a de cela plusieurs années, la communauté scientifique avait pour idée que les conclusions s'appliquant à l'anglais étaient généralisables pour ce qui est des autres langues, malgré le fait que chaque langue possède ses propres caractéristiques syntaxiques, caractéristiques qui peuvent jouer un rôle majeur sur les conclusions applicables à une étude. Il a déjà été proposé que l'analyse de la structure de la langue obéisse à des principes de base qui s'appliquent à toutes les langues. En 1990, des validations inter-langues ont été réalisées par le biais d'études basées sur des comparaisons de cette nature, dans le but de contrecarrer une hypothèse d'universalité applicable à toutes les langues. Il en est ressorti que les particularités associées aux langues ne sont pas universelles; elles dépendent des caractéristiques syntaxiques et des particularités de chaque langue (Nicolas, 2003). En outre, selon Morgenstren (2009), les façons de parler concernant la description des relations spatiales diffèrent selon les langues. De plus, des individus parlant une langue particulière recourent à des prépositions pour exprimer les choses, alors que d'autres personnes, qui ne parlent pas la même langue, utilisent d'autres moyens. Également, d'après Hickmann, Hendriks et Roland (1998), des différences inter-langues sont présentes dans la vitesse d'acquisition de la langue. Les chercheurs mentionnent aussi que des études ayant comparé l'anglais et d'autres langues montrent que « $[\ldots]$ les processus de focalisation et 
d'encodage grammatical vs lexical diffèrent d'une langue à l'autre, aussi bien chez le jeune enfant que chez l'enfant plus âgé [...] » (Hickmann et al., 1998 : 119). Les résultats des études anglophones et francophones peuvent donc s'opposer selon plusieurs aspects. Ici, il est possible de lier ces affirmations avec les études anglophones (Varnhagen et al., 2009; Tagliamonte, 2007) recensées sur le sujet étudié. En effet, les conclusions de ces études montrent que l'anglais n'a pas à craindre l'avènement du clavardage. Par contre, les différences inter-langues pourraient peut-être faire en sorte que les conclusions d'une étude francophone soient différentes, d'où l'intérêt de se pencher sur l'étude de l'incidence du clavardage sur la maîtrise du français écrit.

Au Québec, une étude empirique visant à étudier l'influence du clavardage sur le français écrit a été réalisée par Lafontaine et al. (2005). L'objectif de cette étude était de comparer entre elles les performances en français écrit des élèves qui utilisent le clavardage comme outil de communication de ceux qui ne l'utilisent pas du tout. Les chercheurs ont déterminé si les élèves clavardent ou non par le biais d'un questionnaire. L'échantillon était constitué de 199 élèves de cinquième secondaire. Dans le but d'analyser l'influence du clavardage, les sujets ont eu à réaliser deux activités, soit une dictée de huit phrases, ainsi que la correction de plusieurs expressions qui ont été puisées selon les codes du clavardage. Les élèves ont été au préalable questionnés sur leurs compétences personnelles en français par le biais d'une autoévaluation, de même que sur leur moyenne générale dans cette matière. Les résultats ont révélé qu'il n'y a pas de différence significative entre les clavardeurs et les non-clavardeurs en ce qui a trait aux éléments évalués cités précédemment, ce qui a permis aux chercheurs d'affirmer que le clavardage n'a pas d'impact négatif sur le français écrit des jeunes de cinquième secondaire.

En France, David et Goncalves ont étudié la langue codée attribuée aux textos. D'après leurs recherches, ils ont établi les différentes modifications qu'apportent les textos à la langue française. À la lumière de leurs études, ils ont fait certaines constatations. Selon eux : 
[...] les élèves du secondaire ne semblent confondre ni les registres de langue dans leurs échanges oraux, ni les registres d'écriture dans leurs devoirs écrits. Et s'ils le font, c'est moins dans la confusion des écritures texto et conventionnelle que dans la non maîtrise sic des formes et normes orthographiques. Les écrits scolaires étudiés par nous, dans des établissements accueillants sic des élèves sociologiquement contrastés, ne permettent pas de déceler des traces d'écriture inventées ou abrégées, analogues à l'écriture texto. Nous assistons en fait à l'émergence d'une digraphie - voire d'une plurigraphie - comparable à celle que nous observons dans les usages oraux de la langue (David et Goncalves, $2007: 8$ ).

Par ailleurs, lorsque les intentions de communication changent, l'écriture subit le même sort. Par exemple, lorsqu'un adolescent écrit à ses amis par l'intermédiaire de son téléphone cellulaire, il écrit d'une façon particulière. Par contre, lorsqu'il communique avec ses parents par le biais de ce même média, l'écriture est différente, puisqu'il ne s'adresse pas à des utilisateurs adolescents. Ces éléments laissent entrevoir que les jeunes sont capables de faire la part des choses et de savoir dans quels contextes ils peuvent utiliser une langue en particulier (David et Goncalves, 2007).

Une étude antérieure réalisée par Smith (1990) a montré que les étudiants conversant par le biais du clavardage dans un cours d'espagnol langue seconde ont mieux performé lors d'une épreuve orale que ceux qui n'avaient pas eu l'occasion de dialoguer par le biais d'Internet. En effet, ces étudiants ont eu des scores plus élevés aux examens oraux que ceux n'ayant pas eu cette possibilité, ce qui démontre que l'utilisation du clavardage a un effet bénéfique sur les performances orales dans le cadre de l'apprentissage d'une langue seconde.

Sur la base de ces résultats, Blake (2009) a réalisé une seconde recherche plus récente, centrée elle aussi sur l'influence du clavardage sur l'oral lors de l'apprentissage d'une langue seconde. Dans le cadre de cette étude, la langue seconde était l'anglais. L'hypothèse de recherche énoncée par Blake était que les participants utilisant le clavardage ont des performances plus élevées en évaluation orale que ceux qui n'ont pas évolué dans cette activité. D'ailleurs, les résultats de Smith (1990) ont été reproduits par la suite par Blake lors de son étude. En effet, les personnes utilisant le clavardage ont bel et 
bien de meilleurs résultats aux tests oraux que ceux ne faisant pas usage de cette nouvelle technologie. L'une des explications donnée à cet effet est que les personnes n'ayant pas eu accès à Internet à des fins de communication ont eu moins d'opportunités de pratiquer leur anglais, comparativement à ceux qui ont pu clavarder durant leurs heures de classe. Nous pouvons alors aisément considérer l'apport du clavardage sur les performances orales lors de l'apprentissage d'une langue seconde comme étant un bienfait associé à ce média électronique.

En somme, les études réalisées sur le clavardage lors des années antérieures démontrent que celui-ci n'est pas nocif pour la langue écrite et qu'il peut même être bénéfique pour l'oral lors de l'apprentissage d'une langue seconde. En revanche, au Québec, une seule recherche a été effectuée sur le sujet, avec une clientèle et une méthodologie différentes de celle de la présente étude. Dans l'ensemble, il demeure que peu de recherches ont été consacrées au phénomène du clavardage malgré son ampleur.

\subsection{DIFFERENCES ENTRE LES SEXES AU NIVEAU DES PERFORMANCES EN ECRITURE}

Lévesque, Lavoie et Chénard (2007) ont étudié les différences académiques entre les élèves de sexe masculin et féminin. Les chercheurs ont ainsi constaté que les garçons ont plus de difficultés d'apprentissage que les filles. Ceux-ci accumulent en effet plus de retard et sont plus nombreux à redoubler et à abandonner l'école. Ces caractéristiques sont encore plus importantes chez les garçons issus de milieux défavorisés. D’ailleurs, l'apprentissage de la lecture et de l'écriture se concrétise moins facilement chez les garçons que chez les filles. De plus, lors des épreuves finales de secondaire cinq au Québec, les filles ont été plus performantes que les garçons (MEQ, 2003). Concernant le retard moyen au primaire et au secondaire, celui-ci était de $17.1 \%$ pour les deux sexes confondus, soit de $20 \%$ pour les garçons et de $14.2 \%$ pour les filles (MELS, 2006). Il est donc possible de remarquer que les garçons sont davantage en retard à l'école que leurs camarades de classe féminines. Selon les statistiques de 32 pays occidentaux incluant le Canada, les filles ont un meilleur 
rendement scolaire que les garçons (CSÉ, 1999). Il importe tout de même de mentionner qu'il n'y a pas de différence au niveau des résultats scolaires entre les deux sexes lors des premières années du primaire. En revanche, dès la sixième année, les filles prennent de l'avance sur les garçons. L'écart se fait ressentir au fil du temps et ce sont les filles qui ont alors les meilleurs résultats scolaires en lecture et en écriture (CSÉ, 1999). Il semblerait également que le nombre de garçons en difficulté d'apprentissage et d'adaptation soit deux fois plus nombreux que le nombre de filles (CSÉ, 1999). Ces constats interrogent la possibilité que les garçons soient plus vulnérables que les filles à l'impact défavorable du clavardage sur la maîtrise du français écrit.

\subsection{CONCLUSION}

Par l'intermédiaire de ce premier chapitre, une recension des écrits complète a été rédigée concernant le sujet à l'étude. Divers éléments ont alors pu être précisés. Le clavardage consiste en un mode de communication basé sur des discussions virtuelles en direct avec une ou plusieurs personnes, qui se démarque par ses aspects de rapidité et d'instantanéité. Le clavardage est très populaire en temps actuel; il est notamment priorisé par les adolescents d'aujourd'hui. Effectivement, un nombre considérable de jeunes clavardent quotidiennement et ce, en utilisant une langue codée. Pour clavarder, les internautes utilisent principalement des programmes de clavardage comme Msn Messenger, de même que des réseaux sociaux, tels que Facebook et Myspace.

Dans la cadre de la problématique, les aspects positifs et négatifs du clavardage ont été mis en évidence. En effet, ce nouveau mode de communication comporte des bienfaits à prendre en considération. Notamment, le clavardage suscite l'envie de communiquer par écrit avec autrui, ce qui fait en sorte que les personnes utilisant cette forme de communication interagissent avec leurs semblables, ce qui est valorisant au niveau social. On peut donc présumer que les jeunes écrivent plus qu'avant. La langue codée est également caractérisée comme étant basée sur un esprit collaboratif et ouvert. Par ailleurs, 
le clavardage permet à la société d'élargir son champ par rapport à la communication écrite et favorise le contact entre individus (Anis, 2003). Le clavardage pédagogique est aussi à prendre en compte, puisqu'il peut apporter plusieurs avantages aux élèves qui en bénéficient. Par exemple, ils ont, par le biais de cette activité pédagogique, la chance de découvrir de nouvelles cultures et de se faire des amis vivant dans un milieu différent, tout en travaillant leur français écrit (Faverjon, 2003). Du côté négatif, il est impossible de passer sous silence le phénomène de cyberintimidation, qui prend de plus en plus d'ampleur au fil du temps. Majoritairement, les jeunes cyberintimidateurs se servent du clavardage pour intimider leurs victimes, ce qui amène que plusieurs adolescents vont se priver du clavardage pour ne pas avoir à affronter leurs bourreaux. Ce phénomène peut nuire au cheminement personnel, social et scolaire des élèves intimidés (Chantelois, 2008).

En ce qui concerne les caractéristiques de la langue codée utilisée dans le cadre de séances de clavardage, tout comme dans les textos, celles-ci sont diversifiées. Tout d'abord, les individus ont tendance à faire appel à des réductions graphiques, c'est-à-dire qu'ils vont couper certaines lettres dans un mot, ce qui fait en sorte que celui-ci se rapproche davantage de l'oral. Par ailleurs, les réductions et transformations avec variantes phonétiques font elles aussi partie intégrante de cette forme de communication. De plus, plusieurs particularités sont à noter concernant la langue codée requise en contexte de clavardage. Notamment, cette langue se caractérise comme étant hétérogène, polyvalente et variable. Les troncations et les onomatopées sont également souvent présentes. Aussi, les 《 smileys », visant à exprimer des émotions par l'intermédiaire d'illustrations, sont souvent utilisés par les adeptes du clavardage. Les éléments de ponctuation sont quasi-absents, mis à part lorsque les internautes désirent mettre l'emphase sur une phrase en particulier. Les traits linguistiques à la base de toute conversation électronique demeurent le souci de rapidité et d'instantanéité des réponses, ce qui veut dire que les individus vont couper des lettres ou des parties de mots dans le but de sauver du temps et d'écrire le plus rapidement possible. De plus, plusieurs vont transgresser les normes de la langue française d'une manière extrême, seulement dans l'objectif de se faire remarquer. Des signes vont également transformer un mot, signes que les personnes n'utilisant pas cette formule de 
communication ne sauraient reconnaître. Par exemple, asv, qui représente «âge, sexe et ville » (Anis, 2003 ; David et Goncalves, 2007).

Plusieurs chercheurs s'entendent pour dire que la langue utilisée lors de séances de clavardage possède plusieurs similitudes avec la langue orale, étant donné que les mots sont écrits comme ils se prononcent. Toutefois, l'une des caractéristiques particulières du clavardage est l'absence d'oralité, puisque la parole n'est pas entendue au niveau sonore. Selon Filatova (2006), nous ne pouvons caractériser les conversations par le biais du clavardage comme étant de véritables conversations, puisque qu'il manque à ces discussions deux éléments majeurs qui font en sorte qu'une conversation en est bien une : la sonorité de la parole et la présence physique de l'interlocuteur.

Par ailleurs, les opinions quant aux effets du clavardage sur la langue écrite sont divergentes. Certains voient le clavardage d'un bon œil et sont d'avis que le français ne doit pas craindre l'essor du clavardage, étant donné que les jeunes d'aujourd'hui sont conscients du fait que le français conventionnel et le français codé utilisé en contexte de clavardage ont deux utilités complètement différentes. Par exemple, Ouellon (2009) ne s'inquiète pas de l'avènement du clavardage. Il le voit même positivement, étant donné que l'utilisation de la langue codée prouve que les jeunes sont capables de s'adapter à l'emploi de deux formes de langue.

En ce qui concerne Waterlot (2008), elle expose pour sa part que le niveau de la maîtrise du français est insuffisant. Par contre, elle mentionne que cette insuffisance du français n'est pas liée au clavardage et que l'ère d'Internet n'est pas considérée comme une menace. Cerisier (2003) met quant à lui en évidence que les jeunes écrivent de plus en plus grâce à l'utilisation du clavardage, ce qui constitue un avantage qu'il ne faut pas négliger.

Toutefois, d'autres professionnels du milieu éducatif sont d'avis qu'il est possible que le clavardage affecte la qualité de la langue française écrite. Notamment, Simard (2002) pense qu'il est normal de s'inquiéter par rapport à cette nouvelle forme de communication et qu'il importe de faire de la prévention à son égard. Par ailleurs, Meney (2007) a fait 
savoir qu'il possède un avis partagé sur le sujet. En réalité, il est capable de cerner les avantages, ainsi que les inconvénients, de l'utilisation du clavardage.

L'un des principaux éléments associés à cette problématique est que la plupart des écrits trouvés sur l'effet du clavardage sur la maîtrise de la langue écrite consistent en des opinions. Effectivement, plusieurs chercheurs et acteurs de l'éducation ont mentionné leur opinion par rapport au clavardage, mais peu d'entre eux l'ont vérifiée. De plus, nous avons pu remarquer que les points de vue recensés sont mitigés, étant donné que des individus touchés de près par le phénomène voient le clavardage d'un œil positif, neutre ou ambivalent, alors que d'autres manifestent une certaine inquiétude quant à son impact sur la maîtrise de la langue.

Concernant les études sur les clavardage, soulignons que Varnhagen et al. (2009) ont réalisé une étude anglophone dont le but était de vérifier l'influence du clavardage sur la langue. Leur méthodologie consistait à effectuer des tests d'épellation à l'aide de mots issus du clavardage. Il a été conclu que les jeunes anglophones qui clavardent sont aptes à épeler de la bonne façon les mots utilisés lors de séances de clavardage, ce qui laisse entendre que le clavardage n'aurait pas d'incidence défavorable sur l'orthographe. Par contre, cela reste à vérifier au niveau francophone écrit, étant donné que les caractéristiques du français diffèrent à plusieurs niveaux de celles de l'anglais. D'autre part, Tagliamonte (2007) a étudié la langue associé au clavardage, toujours en anglais. Elle a pu constater lors de ses recherches que les abréviations en situation de clavardage représentent seulement $2,4 \%$ de l'ensemble du vocabulaire conventionnel anglophone, ce qui en constitue une toute petite partie. Selon elle, les jeunes utilisateurs se servent généralement davantage de mots courants, ce qui contribuerait au fait qu'ils n'utilisent pas assez les mots issus de la langue codée pour qu'ils puissent nuire à leur écriture en général. En somme, du côté anglophone, le clavardage n'aurait pas d'incidence défavorable sur la langue. Par ailleurs, Lafontaine et al. (2005) ont vérifié l'influence du clavardage sur le français écrit des élèves de cinquième secondaire et ont abouti à la conclusion que le clavardage n'a pas d'effet néfaste sur le français écrit des élèves de ce niveau. 
Smith (1990) et Blake (2009) ont pour leur part centré leurs recherches respectives sur l'influence du clavardage sur l'oral lors de l'apprentissage d'une langue seconde. La conclusion de ces études est que les étudiants participant à des sessions de clavardage performent mieux lors d'une évaluation orale que ceux n'y ayant pas participé, ce qui vient confirmer les apports du clavardage sur la langue orale dans le cadre de l'apprentissage d'une langue seconde. Donc, le clavardage serait même favorable à l'apprentissage de l'oralité d'une langue seconde.

\subsection{PROBLEME DE RECHERCHE}

En somme, malgré le fait qu'il s'agit d'un sujet dont le questionnement est récent, quelques recherches et sites Internet sont consacrés au clavardage et à la langue y étant associée. En revanche, le problème est que très peu d'études ont vérifié si des liens peuvent être établis entre le clavardage et la maîtrise du français conventionnel écrit en contexte scolaire. En fait, à notre connaissance, la seule étude qui a vérifié spécifiquement l'influence du clavardage sur la maîtrise du français écrit au Québec est celle de Lafontaine et al. (2005), qui s'est déroulée avec des jeunes de cinquième secondaire et dont la clientèle et la méthodologie sont différentes de la nôtre. Mis à part pour cette dernière étude, les autres recherches sur l'impact du clavardage sur la maîtrise de la langue portent sur une autre langue que la langue française, sont centrées sur la maîtrise de la langue orale, et non écrite, ou se sont concentrées sur l'écriture par textos. Les études sur le clavardage sont donc relativement récentes et à ce jour peu nombreuses au Québec. De plus, davantage d'opinions que d'études ont été trouvées aux fins de cette recension des écrits, alors qu'il est essentiel de se baser sur des éléments scientifiques afin d'établir l'impact d'un phénomène sur un autre. Ainsi, la question à la base de ce mémoire est la suivante : quelle est l'influence du clavardage sur la maîtrise du français écrit des élèves du premier cycle du secondaire? L'objectif de la recherche, quant à lui, est de vérifier l'influence du clavardage sur la maîtrise du français écrit des élèves du premier cycle du secondaire. Le cadre 
théorique qui suit tentera d'apporter des éléments de réponse à la question et à l'objectif de recherche. 



\section{CHAPITRE 3}

\section{CADRE THÉORIQUE}

\subsection{INTRODUCTION}

La psychologie cognitive, de même que la théorie du traitement de l'information, constitueront l'assise du cadre théorique de ce mémoire de maitrise. Il est pertinent de s'attarder au fonctionnement cognitif de l'élève dans le but de vérifier si l'apprentissage et l'utilisation de la langue codée employée en contexte de clavardage peuvent interférer avec les connaissances qu'il possède par rapport à la langue française écrite. Dans ce chapitre seront précisés différents aspects de la psychologie cognitive, dont entre autres la métacognition, le transfert des apprentissages, les variations linguistiques ainsi que l'apprentissage de l'écriture. De plus, des liens entre le clavardage et la façon dont apprend l'élève selon la psychologie cognitive seront mis en évidence.

\subsection{PSYChOLOGIE COGNITIVE}

Puisque la psychologie cognitive est au centre du cadre théorique de la présente recherche, il est approprié de la décrire brièvement. La psychologie cognitive a vu le jour entre 1955 et 1960. En effet, en 1956 a eu lieu un symposium où étaient réunis des linguistes et des psychologues. La rencontre s'est déroulée au «Massachusett Institute of Technology ». Ces scientifiques se sont rencontrés dans le but de discuter de leurs recherches respectives et de l'évolution des recherches sur la psychologie cognitive. D'ailleurs, en 1960, l'Université Harvard a fondé un centre d'études cognitives, dont les pionniers étaient Bruner et Miller. Cette forme de psychologie s'intéressant aux fonctions psychologiques et cognitives lors de sessions d'apprentissage fait maintenant partie intégrante d'un vaste secteur de la psychologie (Cadet, 1998). 
Deschênes (1991) a élaboré une définition de la cognition en tenant compte des caractéristiques de la psychologie cognitive.

Il définit la cognition comme un ensemble d'activités mentales qui permettent la réalisation de différentes tâches cognitives, telles que lire, écrire un texte, mémoriser, résoudre un problème; les habiletés de gestion de la mémoire, de représentation des connaissances, d'abstraction, de résolution de problèmes, de transfert, sont des habiletés cognitives. II indique ensuite que l'activité métacognitive renvoie à l'observation et l'analyse de la démarche cognitive pour assurer le contrôle du processus en cours (Deschênes, 1991, dans Portelance, $1998: 49$ ).

La psychologie cognitive concerne donc le traitement de l'information. L'apprentissage en est le terme central et la personne doit sélectionner les informations ayant une importance pour la tâche qu'elle doit effectuer. Les connaissances antérieures sont primordiales, étant donné que l'apprenant doit faire des liens entre les nouvelles informations et les connaissances qu'il possède déjà par rapport au même sujet (Tardif, 1992). «La psychologie cognitive examine comment les individus perçoivent, apprennent, se souviennent, et pensent l'information » (Sternberg, $2007: 22$ ).

Concernant le clavardage, l'influence de la psychologie cognitive facilitera la compréhension de ce concept par rapport à la réflexion de l'élève au niveau cognitif, autant en ce qui concerne les stratégies d'apprentissage que la façon dont il utilise les connaissances acquises selon les différents contextes. Dans le cas présent, les différents contextes concernent principalement les contextes scolaires et sociaux. Par exemple, lorsque l'élève écrit un texte à l'école, il doit l'écrire en utilisant une langue qui respecte les normes de la langue française, donc en utilisant un français conventionnel. Par contre, lorsqu'il clavarde avec ses amis, il peut utiliser la langue codée relative au clavardage, tant qu'il est capable de s'en départir de retour en classe ou en contexte formel d'écriture. En somme, il est pertinent d'approfondir les éléments de la psychologie cognitive par rapport au sujet. Ces explications nous permettront de mieux comprendre les particularités du clavardage en lien avec l'apprentissage du français conventionnel. 


\subsection{Strategies Metacognitives et COGNitives}

La métacognition est une composante de la psychologie cognitive et constitue un élément central de ce cadre théorique. Elle peut être définie comme un concept clé permettant de distinguer les personnes en réussite de celles qui sont en difficulté d'apprentissage. La métacognition est constituée de deux composantes: les connaissances sur la façon d'apprendre et la capacité de l'apprenant à les réguler (Doudin et Martin, 1998).

Selon Flavell (1985), la métacognition concerne la connaissance qu'ont les êtres humains par rapport à leurs processus cognitifs, ce qu'ils produisent, de même que tout ce qui y est relié. Elle vise à mener une réflexion particulière dans un processus cognitif qui se déroule au moment même et d'en faire l'évaluation. De plus, l'autoévaluation est un des points culminants de la métacognition. Peu importe le contexte, le but premier de l'apprenant est d'être capable de verbaliser sur le sujet travaillé. Il doit aussi être conscient de ses apprentissages et le prouver lors des évaluations. S'il réussit ses évaluations, il sera établi qu'il possède de bonnes aptitudes au niveau métacognitif, puisqu'il aura su gérer adéquatement ses connaissances lors de la réalisation d'une tâche (Flavell, 1985).

Pour sa part, Brown (1987) précise que la métacognition concerne les mécanismes de régulation et de contrôle associés au fonctionnement cognitif. Par le mot mécanisme, il fait référence à des activités qui permettent de guider et de réguler l'apprentissage et le fonctionnement cognitif. De même, les mécanismes métacognitifs ont plusieurs fonctions : la planification, où l'apprenant cherche comment il peut résoudre un problème, ainsi que les stratégies qui pourront l'aider à le faire, la prévision, où la personne prévoit le résultat de la tâche, et le guidage, où la révision et l'organisation des informations se déroulent. Les deux dernières fonctions sont le contrôle des résultats, lors duquel l'individu évalue son résultat en se fiant à l'objectif de départ associé à la résolution du problème, de même que le transfert, le maintien et la généralisation d'une stratégie qui pourrait être applicable à d'autres problèmes. 
Un élève ayant de bonnes habiletés métacognitives devrait manifestement être capable de différencier les formes d'écriture qu'il doit utiliser selon les contextes, c'est-àdire lorsqu'il est en classe, où il doit utiliser la langue écrite conventionnelle, ou en situation de clavardage, lors de laquelle il va majoritairement recourir à une langue codée. En effet, les aptitudes métacognitives de l'élève feraient en sorte qu'il connaitrait les moyens qui lui permettent de guider et de réguler ses apprentissages afin de réussir une tâche. Par ailleurs, en contexte d'écriture formel, les capacités métacognitives de l'élève lui permettraient, entre autres, de prévoir le résultat de son travail, d'aménager adéquatement les informations mentionnées dans son texte, ainsi que de le réviser pour diminuer le nombre d'erreurs. Ainsi, cette distinction contribuerait positivement aux résultats obtenus lors de ses évaluations. Par contre, il est nécessaire de se questionner concernant les élèves ayant de moins bonnes aptitudes métacognitives, soit les élèves en difficulté d'apprentissage. Selon Tardif (1992), la métacognition a le pouvoir de différencier les élèves qui éprouvent des difficultés de ceux qui sont plus performants. D'ailleurs, de l'avis de Bazin et Girerd (1997), une déficience au niveau métacognitif peut faire en sorte que certains élèves ne se situent pas en situation de réussite. Ces deux auteurs mentionnent que les élèves en difficulté ont tout simplement un fonctionnement cognitif moins développé que les élèves performant davantage. En effet, les jeunes ayant des lacunes scolaires trouvent la tâche plus complexe lorsqu'ils doivent expliquer et verbaliser leur fonctionnement cognitif. L'élève en difficulté d'apprentissage doit être capable de cerner les éléments facilitant sa réussite, de même que de se donner les moyens pour les utiliser et les rendre disponibles, même s'il s'agit d'une tâche qui est particulièrement difficile pour lui. À ce moment, l'enseignant peut lui apporter son aide, en lui faisant prendre conscience des stratégies favorisant sa réussite scolaire. Voici les éléments dominants présentés par les auteurs:

Tous les enfants, et probablement tous les individus, possèdent un répertoire cognitif qui ne demande qu'à s'enrichir. Par une activité pédagogique adaptée, on peut y contribuer. Le travail scolaire, tel qu'il est présenté habituellement, ne met pas les enfants en situation d'égalité face à la réussite d'une tâche car tous n'ont pas de méthode clairement définie. La différence se situe seulement au niveau des 
méthodes et des moyens employés pour utiliser les stratégies (Bazin et Girerd, 1997 : 87).

Doly (1997) précise que des recherches sur des élèves en difficulté d'apprentissage ont aussi établi que les échecs de ces élèves sont dus à une déficience d'ordre métacognitif. En effet, ces élèves peuvent avoir les mêmes connaissances et compétences que les élèves plus forts, mais ne tout simplement pas savoir comment s'en servir. Leurs méthodes de travail sont elles aussi déficientes, de même que leur degré de motivation, ce qui contribue à leurs échecs répétés. Les connaissances leur sont manquantes, ou bien impossibles d'accès, lorsqu'ils sont appelés à réaliser une tâche. Par contre, ces élèves ont tendance à croire que leurs échecs ont été causés par des éléments hors de contrôle, ce qui fait en sorte qu'ils abandonnent rapidement et dépendent entièrement de l'aide qui leur est apportée par les autres.

Alors, si ces élèves ont de la difficulté à réguler leurs apprentissages et à autoévaluer leurs performances scolaires, peut-être seront-ils moins aptes à distinguer les deux formes d'écriture (langue conventionnelle et langue codée) comparativement aux élèves ayant des aptitudes métacognitives plus développées. Grangeat (1997) explique que les élèves sont en réussite lorsqu'ils sont aptes à appliquer, réutiliser et transférer leurs apprentissages dans divers contextes. Donc, les élèves performants seraient pour leur part capables de se départir de la langue codée en contexte d'écriture formel. Ils pourraient en effet bien cerner les stratégies d'apprentissage à utiliser pour favoriser leur succès scolaire.

Chouinard, Plouffe et Roy (2004) ont travaillé sur les différences entre les élèves ayant des résultats scolaires acceptables de ceux en difficulté d'apprentissage au regard de l'approche cognitive de la motivation à apprendre. Il en est ressorti que les élèves en difficulté ont tendance à rechercher des buts d'évitement lors des différentes tâches scolaires, ce qui contribue au fait que ceux-ci vont tout faire pour écarter la négativité associée à l'échec, puisque l'échec agit directement sur leur estime personnelle. Ils cherchent également à fournir le minimum lorsqu'ils sont en classe, en visant tout juste la note de passage. Souvent, la perception de ces élèves est qu'ils ne sont pas capables de 
réussir à l'école, ce qui fait en sorte qu'ils voient négativement le rôle de leurs efforts dans leur succès scolaire (Chouinard, 1998). Ils ne sont pas conscients que leurs difficultés sont issues non pas d'habiletés déficientes, mais de stratégies manquantes (Wong, 1991). Comme le précisent Chouinard, Plouffe et Roy (2004), les élèves en difficulté sont différents des élèves plus forts. Ces premiers ne désirent pas se démarquer par l'intermédiaire de leurs résultats scolaires, en plus d'avoir un niveau de persévérance scolaire peu élevé. Ils accordent peu d'importance à leurs matières scolaires. Toutes ces explications nous permettent de comprendre les différences entre les élèves qui réussissent et les élèves en difficulté d'apprentissage, pour qui les échecs sont souvent répétitifs.

Dans le cadre de notre étude, l'expérimentation se déroule avec des jeunes de groupes réguliers. Les éléments mentionnés précédemment démontrent qu'il est prévisible que ceux-ci auront pour la plupart de bonnes aptitudes métacognitives. Par contre, de nos jours, il y a souvent des élèves en difficulté dans les groupes réguliers, ce qui fait en sorte qu'il est nécessaire de s'attarder à leur réalité. Il est alors possible de présumer que la métacognition influence la capacité de l'élève à transférer ou non les mots du clavardage dans ses écrits scolaires.

Dans un autre ordre d'idées, le système cognitif comprend la globalité des stratégies cognitives, stratégies qui ont pour fonction d'agir sur l'environnement immédiat et de soutenir l'aptitude de la personne à utiliser les informations apprises. Celle-ci doit par conséquent recourir aux connaissances acquises de manière utilitaire et fonctionnelle, ce qui ne peut être réalisé sans que les stratégies cognitives n'aient été développées. Ces stratégies cognitives peuvent être définies comme étant les actions de nature intellectuelle que réalise l'apprenant lors d'une tâche quelconque. Lorsque l'élève est capable de gérer correctement sa démarche d'apprentissage dans le cadre d'une activité, il est possible de déduire que ses résultats, de même que ses apprentissages, seront plus révélateurs. Ils démontreront davantage ses véritables capacités scolaires. Par ailleurs, il est du rôle de l'institution scolaire de favoriser le développement des stratégies cognitives, étant donné que ces stratégies facilitent les interactions sociales. Par le fait même, l'apprenant est 
capable d'évaluer la qualité de ses propres interactions par le biais de ses stratégies cognitives (Tardif, 1992). Il peut s'agir à la fois des interactions écrites, par exemple lors de communications par le biais du clavardage, qu'orales:

L'élève doit pouvoir utiliser fonctionnellement les connaissances acquises et, à cet effet, il doit avoir développé un ensemble de stratégies cognitives et métacognitives qui lui permettront de poser les actions appropriées dans les diverses tâches qu'il est appelé à réaliser (Tardif, $1992: 44$ ).

Il posera les bonnes actions écrites dans tous les contextes, ce qui inclut la communauté virtuelle. Nous pouvons lier ces affirmations aux élèves étant capables de distinguer quand ils doivent utiliser la langue codée de quand ils ne doivent pas le faire. Le système cognitif permet à ces élèves de savoir quand il est nécessaire d'utiliser leurs apprentissages; il s'agit ici de l'apprentissage de l'écriture conventionnelle utilisée en contexte scolaire et de celle associée à la langue codée qui est requise lorsqu'ils clavardent avec leurs pairs.

Par ailleurs, le fait d'être capable de prendre conscience de son propre fonctionnement cognitif constitue une preuve d'intelligence; le sujet doit être habilité à bien le comprendre, ce qui fait en sorte qu'il saura quelles stratégies favorisent son apprentissage lorsqu'il doit apprendre un élément nouveau. Par ailleurs, être capable d'autoévaluer ses performances et ses stratégies est essentiel si l'on désire bien cerner comment fonctionne le cerveau cognitivement. Cela permet à l'apprenant de mieux apprendre et de réussir davantage. Un élève autonome dans ses apprentissages doit être susceptible, par lui-même, de construire des connaissances sur son propre fonctionnement cognitif et celui d'autrui, d'utiliser ses fonctions mentales efficacement, de même que de recourir à un répertoire où sont logées les différentes stratégies utiles à la réalisation de tâches scolaires et courantes. En résumé, être apte à utiliser ces stratégies favorise l'autonomie en contexte scolaire (Nicolas, 2003), de même qu'en contexte de clavardage. Si l'élève est autonome au niveau cognitif, il sera attentif à la façon dont il fonctionne lorsqu'il apprend ou écrit. 
Tout compte fait, les habiletés et les stratégies métacognitives et cognitives de l'élève contribuent grandement à la réalisation d'une tâche d'apprentissage, étant donné qu'elles favorisent sa réflexion lorsqu'il est en processus rédactionnel ou en situation de clavardage. En outre, les élèves en difficulté d'apprentissage, qui sont nombreux à être intégrés en classe régulière, ont pour la plupart des aptitudes métacognitives moins développées que les élèves plus performants. Ainsi, à l'opposé des élèves plus forts, il pourrait être possible que ces élèves aient davantage de difficulté à faire la distinction entre les mots attribués à la langue codée qu'ils utilisent lorsqu'ils clavardent, et le français conventionnel, qu'ils doivent employer lors d'une tâche d'écriture en contexte formel, d'autant plus que le français conventionnel peut être difficilement maîtrisé par ces élèves. Par conséquent, ils pourraient être plus à risque d'utiliser la langue codée en contexte scolaire.

\subsection{Connaissances}

La façon dont les connaissances sont organisées et représentées dans la mémoire joue un rôle primordial. Effectivement, lorsque les connaissances sont bien organisées, il est plus facile pour l'individu de lier de manière appropriée les nouvelles informations avec celles qu'il connaît déjà, ce qui fait en sorte que l'utilisation de ses connaissances est favorisée. Il est également plus simple pour l'individu d'utiliser ses connaissances selon les divers contextes (Tardif, 1992). Ces affirmations expriment donc que des connaissances bien organisées dans le cerveau favorisent leur utilisation selon les deux contextes qui nous intéressent.

Il faut prendre en considération les types de connaissances, qui sont classées comme suit: les connaissances déclaratives, les connaissances procédurales, ainsi que les connaissances conditionnelles. Ces connaissances sont liées entre elles de manière systématique; elles sont également très bien coordonnées sous forme de schéma dans la mémoire de l'être humain. 
Les connaissances déclaratives s'attardent seulement aux idées nécessaires. Plus clairement, elles sont les connaissances que possède une personne sur un objet ou une notion théorique (Tardif, 1992). Nadeau (1995) précise que ces connaissances sont effectivement reliées au domaine théorique et qu'il s'agit des connaissances dont la mémorisation est possible.

Boudreau (1995) met en évidence que les connaissances de nature déclarative sont requises lorsque les apprenants sont en processus d'écriture. Elles sont notamment à la base des concepts de pertinence syntaxique, convenance lexicale, relations morphosyntaxiques, ainsi que du respect de l'orthographe d'usage (Roy, Lafontaine, Boudreau et Viau, 1992). Par contre, les connaissances déclaratives ont également d'autres utilités, surtout lorsque l'élève rédige un texte; elles sont liées aux stratégies cognitives que l'élève connaît et qui contribuent à la rédaction d'un texte, comme la planification, la rédaction et la révision du texte (Boudreau, 1995). Voici ce que précise l'auteur à ce sujet :

Nous convenons, par ailleurs, avec Ericsson et Simon (1984) que les processus cognitifs nécessairement impliqués dans la réalisation d'une tâche s'expriment selon une séquence de procédures intériorisées qui sont successivement adaptées, selon les besoins et les contraintes rencontrées, par traitement de l'information disponible en mémoire à long et à court terme. Pour être intériorisées, ces procédures doivent d'abord être connues et apprises, et leur intériorisation ne sera révélée que par le recours spontané aux stratégies concernées en production de texte (Boudreau, $1995: 76$ ).

Pour ce qui est du deuxième type de connaissances, soit les connaissances procédurales, celles-ci concernent les procédures et les stratégies d'utilisation des connaissances déclaratives de tous les niveaux (Boudreau, 1995). Elles désignent la forme productionnelle de la mémoire à long terme, ce qui fait en sorte que les apprenants doivent réaliser des productions. En fait, une condition à la base de l'apprentissage fait en sorte que l'apprenant réalise plusieurs actions en différentes séquences. Les actions constituent par le fait même une procédure. Les connaissances procédurales peuvent être caractérisées comme étant des savoir-faire (Tardif, 1992). En l'occurrence, en lien avec ce mémoire, le savoir-faire requis est l'action d'écrire. 
Les connaissances conditionnelles constituent le plus complexe et le troisième type de connaissance. Ces connaissances consistent en des savoirs reliés aux conditions d'utilisation d'une notion apprise, de même qu'aux raisons pour lesquelles la connaissance est requise lors de la réalisation d'une tâche. Lorsque le sujet met en commun ses connaissances, les connaissances conditionnelles font en sorte qu'il est davantage habile pour ce qui est de créer des liens entre les connaissances qu'il possède déjà et ce qu'il est en train d'apprendre, ce qui fera en sorte qu'il sera plus à l'aise lorsqu'il devra transférer ses apprentissages dans un nouveau contexte (Tardif, 1992). En fait, Nadeau (1995) souligne que les connaissances conditionnelles agissent comme déterminant pour le transfert des apprentissages. En effet, les connaissances conditionnelles concernent l'application des connaissances, dans le même contexte ou dans un contexte autre. Tardif (1992) a ressorti des exemples de connaissances conditionnelles. En voici quelques-uns :

- un élève qui a pour tâche de trouver le participe passé dans une phrase particulière;

- un élève qui doit reconnaître, parmi deux textes de genres différents, lequel des deux est un texte informatif;

- un élève qui doit estimer le résultat d'une équation en mathématique.

Comme le met en évidence Nadeau :

[...] les connaissances conditionnelles concernent les connaissances requises pour savoir quand avoir recours aux autres connaissances. Il s'agit ici de connaître les conditions d'application de telle ou telle connaissance, et surtout de savoir reconnaître ces conditions dans une situation nouvelle (Nadeau, 1995 : 35).

À cet égard, en ce qui concerne le clavardage, l'élève qui écrit un texte en classe, mais qui passe de nombreuses heures par semaine à clavarder, devrait reconnaître les conditions d'application de ses connaissances, c'est-à-dire reconnaître les différents contextes (contexte de clavardage et contexte scolaire) dans lesquels il utilisera soit la langue codée, soit le français conventionnel.

La métacognition peut ainsi être associée aux connaissances conditionnelles. En effet, pour revenir à ce qui a été mentionné précédemment, les habiletés métacognitives des 
apprenants pourraient peut-être avoir une incidence sur la capacité du sujet d'actualiser efficacement ses connaissances conditionnelles.

Selon Fayol (1991), il est incontestable que la psychologie cognitive permet de bien comprendre les réflexions de l'individu lorsqu'il est en processus d'écriture. Il rappelle que cette forme de psychologie est centrée sur la façon dont les savoirs sont organisés, ainsi que la manière dont se déroule leur acquisition. Les conditions, la gestion, ainsi que le contrôle des savoirs, sont également reliés à la psychologie cognitive. Lorsque l'élève doit rédiger un texte en classe, il doit aller chercher dans son bagage de connaissances déclaratives celles qu'il juge nécessaires. Ces connaissances sont variées, c'est-à-dire qu'elles peuvent s'appliquer autant au code langagier (accord des verbes) comme elles peuvent être reliées aux connaissances que possède l'élève par rapport au processus rédactionnel (élaboration du texte: introduction, développement et conclusion). Par ailleurs, les connaissances procédurales, qui concernent la façon dont les connaissances sont utilisées (comment faire), sont également au centre du processus d'écriture, étant donné que l'élève doit souvent réfléchir à la façon dont s'articule son texte, en appliquant et en mettant en opération ses connaissances. Pour leur part, les connaissances conditionnelles constituent le type de connaissances le plus complexe, étant donné que l'élève doit aller puiser dans son répertoire de connaissances celles qu'il doit utiliser selon les différents contextes.

Il faut donc considérer que les trois types de connaissances, soit les connaissances procédurales, déclaratives et conditionnelles, sont à la base du processus d'écriture. En contexte de clavardage, les connaissances déclaratives et procédurales concernant l'écriture sont moins de mise qu'en situation d'écriture de textes formels en contexte scolaire. Étant donné que les élèves clavardent pour se divertir, ils vont manifestement moins recourir à leurs connaissances déclaratives et procédurales relevant des normes de la langue française; en effet, ils n'en ont pas besoin, puisque leur écriture n'a pas une utilité ciblée. En situation de clavardage, les connaissances les plus sollicitées sont les connaissances conditionnelles, qui elles, permettent aux apprenants de distinguer les deux contextes d'écriture (contexte de clavardage et contexte scolaire) et les langues qui y sont requises, soit la langue codée et la 
langue écrite conventionnelle. Puisqu'il s'agit de connaissances de haut niveau, et appuyées par la métacognition, elles seraient surtout l'apanage des apprenants performants qui d'ailleurs, peut-on présumer, maîtrisent bien les connaissances déclaratives et procédurales du français conventionnel, ce qui n'est peut-être pas le cas des élèves en difficulté.

\subsection{MEMORISATION}

Il est important d'aborder la mémoire, étant donné que l'élève doit y conserver les mots appris depuis le début de sa scolarisation, mots qui lui permettent ensuite d'écrire en contexte de clavardage et en contexte scolaire. La mémoire permet le traitement et la rétention des informations chez les individus. La rétention est caractérisée comme étant le «processus par lequel la mémoire à long terme préserve un apprentissage de façon à ce qu'il puisse être localisé, identifié et rappelé avec précision dans l'avenir » (Sousa, 2002: 91).

Débutons par définir la mémoire de travail, qui est également nommée mémoire à court terme. Celle-ci permet le traitement conscient des informations venant de l'environnement et captées par les récepteurs sensoriels, et les met en lien avec des connaissances antérieures récupérées pour l'occasion de la mémoire à long terme, de manière à les traiter et à leur donner du sens. En fait, le cerveau reçoit les éléments venant de l'environnement et les sens décident si ces éléments seront rejetés ou acceptés. Si le cerveau accepte ces éléments, ceux-ci sont ensuite traités. Ce traitement se fait dans la mémoire de travail. Cette dernière donne la possibilité à l'être humain de maintenir des informations durant plusieurs secondes et minutes. Toutefois, ces informations doivent être rappelées, sinon elles disparaîtront (Sousa, 2002).

La mémoire de travail est directe et se démarque par le fait qu'elle agit immédiatement. Tel que le mentionne Gagné (1985), la mémoire de travail est grandement impliquée dans les activités d'enseignement et d'apprentissage. En effet, l'élève y a recours 
lorsqu'il doit réaliser une tâche. Tardif (1992) précise que la tâche elle-même est toujours résolue dans la mémoire de travail, étant donné que cette mémoire est celle qui est responsable de l'interaction des informations et des connaissances issues de l'environnement et de la mémoire à long terme entre elles, ce qui permet ensuite d'expédier le résultat de la tâche vers la mémoire à long terme, où il est mis en réserve de manière permanente.

Lorsque la mémoire de travail est abordée, il est pertinent de prendre en considération les unités de traitement de l'information incluses dans cette mémoire, qui sont au nombre de sept. L'unité peut contenir un seul ou plusieurs concepts ayant des liens entre eux. En effet, d'après Tardif (1992), les études antérieures ont démontré que les connaissances sont liées entre elles. Lorsqu'elles sont requises dans la mémoire de travail, un réseau de concepts peut alors occuper une seule unité. Ici, la distinction entre les apprenants novices et experts favorise la compréhension des unités de travail. L'apprenant novice possède moins d'informations dans chaque unité, comparativement à l'apprenant expert. Tardif précise que l'apprenant expert possède par unité de travail un ensemble de connaissances davantage développées et liées entre elles. Cela fait en sorte que cet apprenant est capable de résoudre une situation-problème de manière particulièrement efficiente. Par exemple, pour un scripteur débutant, le fait de se concentrer sur la calligraphie peut occuper une seule unité de travail. Par contre, pour un scripteur expert, son niveau de concentration sur la calligraphie, ainsi que d'autres éléments concernant la tâche, par exemple les stratégies relatives au processus rédactionnel, peuvent être inclus dans une seule et même unité de travail, ou même en occuper aucune si elles sont automatisées, c'est-à-dire qu'elles passeront directement de la mémoire à long terme au générateur de réponses, tel que nous le verrons plus en détail au point 3.6.

De plus, la mémoire de travail peut se retrouver en condition de surcharge lorsque trop d'informations tentent d'être traitées en même temps dans cette mémoire. Dans ce cas, elle doit éliminer les informations les moins importantes pour en intégrer de nouvelles, ce qu'elle fait automatiquement (Tardif, 1992). 
La mémoire à long terme, en ce qui la concerne, fait en sorte que l'individu garde en mémoire les éléments les plus importants et les plus marquants de sa vie. Cette mémoire contient aussi les connaissances antérieures de l'élève. Il y a deux sortes de mémoire à long terme. Tout d'abord, la mémoire épisodique concerne les souvenirs et les expériences personnelles de l'individu, alors que la mémoire sémantique contient les concepts et les connaissances universelles. Dans la mémoire sémantique, les connaissances sont organisées en réseaux, c'est-à-dire que des relations sont créées entre les connaissances, ce qui facilite le caractère fonctionnel de l'utilisation et de la récupération de ces dernières (Tardif, 1992).

L'autorépétition est une autre stratégie importante de la mémoire à long terme et est particulièrement utile lors de l'apprentissage de la lecture et de l'écriture. Effectivement, en répétant plusieurs fois, il est plus facile pour une personne d'emmagasiner l'information qu'elle cherche à apprendre. L'information reste alors active (Lindsay et Norman, 1980). En plus de répéter, il est pertinent que l'élève lie les informations apprises avec ses connaissances antérieures, ce qui lui permettra de donner du sens à ses apprentissages et favorisera la mémorisation.

Par rapport au sujet de la présente l'étude, le questionnement se fait surtout ressentir au niveau de la mémorisation des mots reliés aux normes françaises. En effet, au fil de leurs années d'apprentissage, les adolescents ont appris de nombreux mots et ont ainsi développé un riche répertoire orthographique. En revanche, lors de sessions de clavardage, les élèves n'utilisent pas la plupart de ces mots, malgré le fait qu'ils les ont en mémoire. Ils décident donc de ne pas y recourir, et ce, de façon volontaire. D'autre part, les deux contextes, soit le contexte de clavardage et le contexte d'écriture formel, diffèrent quant à l'utilisation qu'ils requièrent de ses unités de traitement de l'information dans la mémoire de travail. En effet, en contexte d'écriture formel, les unités de travail peuvent être remplies à pleine capacité. Par exemple, elles peuvent être remplies par les connaissances déclaratives attribuées au code langagier, par les connaissances procédurales associées à la mise à l'écrit et par les connaissances sémantiques concernant le sujet abordé dans le cadre de la rédaction. Il demeure qu'en situation de clavardage, le contexte est différent et est moins formel que 
lorsque l'apprenant rédige un texte en classe; le sujet clavarde pour communiquer de façon interactive et pour se détendre. À ce moment, les unités de travail seront moins remplies qu'en contexte d'écriture formel, puisqu'il n'est pas nécessaire de les utiliser pleinement en clavardant. L'individu ne souhaite pas en faire une activité cognitive soutenue et remplir à pleine capacité les unités de sa mémoire de travail, ce qui pourrait expliquer la création de la langue codée pour répondre à ce besoin.

Nous savons que les adolescents d'aujourd'hui clavardent en utilisant une langue complètement différente de la langue conventionnelle attribuée aux normes de la langue française. À force de répéter l'utilisation de ces mots issus de la langue codée, ils y sont plus habitués et les ont mémorisés. Ils possèderaient deux mémorisations du même mot, celui de la langue codée et celui correspondant au français conventionnel. Ils semblent donc avoir développé les deux formes de langage écrit et les utiliser successivement.

Pour terminer, il importe de rappeler que la mémoire de travail, qui permet le traitement des informations, et la mémoire à long terme, où est inclus l'ensemble du vocabulaire de l'apprenant, font en sorte que l'apprenant finit par mémoriser les mots codés attribués au clavardage. Il reste simplement à déterminer s'il sera capable de différencier en tout temps les deux formes d'écriture.

\subsection{Automatisation des CONNAISSANCES}

Selon Tardif (1992), l'automatisme est le passage immédiat d'une connaissance issue de la mémoire à long terme dans la direction du générateur de réponses, sans passer par l'intermédiaire de la mémoire de travail. Tardif précise que dans l'ensemble de la mémoire, un espace est prévu pour la réponse anticipée. Cette future réponse est incluse dans la mémoire de travail et remplit moins d'une unité, ce qui fait en sorte qu'elle prend peu de place dans la mémoire de l'être humain. En revanche, cela est différent pour les connaissances automatisées de natures conditionnelles et procédurales. Dans ce cas, la connaissance présagée est conduite de la mémoire à long terme vers le générateur de 
réponses, sans transiter par la mémoire de travail (Tardif, 1992). Tardif mentionne: «Automatiser des connaissances procédurales et conditionnelles est donc un excellent moyen pédagogique pour libérer de l'espace dans la mémoire de travail » (Tardif, 1992 : 174).

Fayol (1991) met en lumière qu'il est préférable, dans le but d'acquérir une connaissance déclarative sur un quelconque sujet, de faire des exercices et non pas uniquement de mémoriser les notions théoriques la concernant. Effectivement, la pratique, qui est rattachée aux connaissances procédurales, favorise l'apprentissage des connaissances déclaratives reliées à l'écriture. Par contre, il développe en expliquant qu'il est tout de même nécessaire de s'attarder aux règles pour réviser ensuite l'écriture des mots. «En résumé, les connaissances procédurales nécessitent un long apprentissage par guidage à partir du déclaratif et / ou par imitation. Leur maîtrise progressive s'obtient par l'exercice ou par les corrections après coup des résultats erronés »(Fayol, 1991: 23). Ainsi, plus l'élève pratique une notion et travaille celle-ci par le biais d'exercices, plus il serait susceptible d'automatiser cette connaissance.

Par rapport au phénomène du clavardage, il importe de préciser que plus l'élève clavarde, plus il pratique la langue codée associée à ce mode de communication. Les jeunes automatiseraient de cette façon plusieurs mots de la langue codée, par exemple $b c q$, pour beaucoup, $p c q$, pour parce que, en $t k$, pour en tout cas, et $p k o i$, pour pourquoi. Hormis quelques mots tels que mentionnés, il n'y a pas vraiment de balise orthographique associée à la langue codée. L'important est surtout que l'élève maîtrise la voie d'assemblage, c'està-dire la correspondance graphème-phonème; il saura alors comment écrire la langue codée et être compris par les autres internautes. Dalpé, Giroux, Lefebvre et St-Pierre (2010) soulignent à cet effet qu'à l'écrit, l'apprenant doit se référer à des procédures d'assemblage et d'adressage. Ces procédures font en sorte que l'élève est apte à traduire les mots sous leur forme sonore (représentation phonologique) en mots écrits. La procédure d'assemblage se met en œuvre de façon graduelle dès les premières relations de l'enfant avec l'apprentissage de l'écriture. Chez les scripteurs débutants, l'enfant doit prioritairement 
faire appel à la procédure d'assemblage lorsqu'il apprend un nouveau mot (Dalpé et al., 2010). Toutefois, on peut se demander si l'automatisation du fonctionnement de l'écriture codée par assemblage n'entraverait pas l'automatisation de la procédure d'adressage, soit les connaissances orthographiques du français conventionnel. Dalpé et al. expliquent que la procédure d'adressage concerne le fait que l'élève accède directement aux représentations orthographiques contenues dans sa mémoire à long terme lorsqu'il doit écrire.

C'est à travers l'expérience liée à la conversion phonographémique et à l'extraction des caractéristiques et régularités orthographiques des mots lus et écrits que le scripteur emmagasine les représentations orthographiques des mots dans son lexique, qu'il les spécifie graduellement et, par le fait même, développe sa compétence en orthographe (Dalpé et al., 2010:39).

D'ailleurs, l'adressage est surtout employé chez les élèves ayant des aptitudes correspondant à celles des scripteurs compétents, dans le sens où leur lexique orthographique est déjà solide comparativement aux scripteurs débutants. Plusieurs mots de la langue codée peuvent s'écrire de différentes façons d'une conversation à une autre. C'est surtout le recours à une écriture grapho-phonétique, au détriment de la procédure d'adressage, qui pourrait ainsi s'automatiser par le biais de la pratique du clavardage.

De même, il est pertinent de s'interroger concernant les élèves en difficulté. Il se peut qu'ils saisissent moins bien les particularités des deux contextes d'écriture que les élèves ayant de plus grandes aptitudes scolaires. Il est possible que ces élèves, tout d'abord, ne maîtrisent pas bien, de par leur degré de difficulté, les connaissances déclaratives et procédurales requises en contexte d'écriture formel. En effet, les élèves en difficulté d'apprentissage peinent à automatiser des stratégies ou des savoir-faire sur la langue écrite, contrairement aux élèves plus forts. En fait, plusieurs élèves en difficulté sont davantage concentrés sur l'action d'écrire en tant que telle; le contenu de leur texte ne constituera donc pas un élément primordial lors de la réalisation d'une tâche d'écriture. Par ailleurs, les élèves en difficulté ne prennent souvent pas le temps de se relire en contexte d'écriture formel et éprouvent des difficultés à savoir comment corriger leur texte lor'squ'ils doivent le faire. Puisque plusieurs éléments sont en jeu lors de l'écriture d'un texte, dont la 
planification, la rédaction et la révision du texte dans son ensemble, il s'agit d'une activité pédagogique complexe pour ces élèves. Lorsqu'ils rédigent un texte, les apprenants doivent mettre en commun plusieurs éléments dans une même tâche relative à l'écriture. Par exemple, en production d'écriture, les élèves doivent rédiger des phrases qui assurent une certaine cohérence. Pour ce faire, ils doivent trouver les bons mots tout en respectant le contexte de la phrase, transférer les notions théoriques relatives aux règles de grammaire apprises lors d'un cours, ainsi que réfléchir à la façon dont les mots sont orthographiés, pour que ceux-ci respectent les règles d'orthographe d'usage. Bref, les élèves en difficulté d'apprentissage ont de la difficulté à automatiser les connaissances sur l'écriture et à les faire interagir efficacement (Debeurme et Lamarche, 2001; Touzin, 2004). Cela contribuerait peut-être au fait que la langue codée du clavardage, de par sa polyvalence et son aspect phonétique, leur siérait mieux. De ce fait, peut-être seront-ils mieux disposés à pratiquer l'écriture en contexte de clavardage qu'en contexte d'écriture formel, ce qui pourrait faire en sorte qu'ils automatiseraient davantage la langue codée que le français conventionnel. Ils seraient alors plus à risque de faire un transfert de la langue codée vers les écrits conventionnels.

En résumé, lorsque les jeunes clavardent régulièrement, ils retiennent bien la façon dont ils doivent écrire la langue codée et ce, par automatisation de celle-ci. En effet, comme le précise Sternberg (2007), il est plus facile de retenir les informations lorsque les apprenants les utilisent par le biais de plusieurs séances d'apprentissage. Nous pouvons ici lier cette affirmation avec la répétition et la pratique, qui favorisent la mémorisation. L'apprentissage de la langue codée associée au clavardage pourrait s'acquérir justement de cette façon, puisque certains jeunes ont tendance à clavarder plusieurs heures par jour.

\subsection{AtTEnTION}

L'attention doit également être considérée. Celle-ci permet aux individus d'être à l'écoute de plusieurs événements en même temps. Toutefois, cela peut être difficile pour 
une personne en contexte social, surtout si elle cherche par exemple à être plus attentive à une conversation plutôt qu'à une autre. L'attention limite l'aptitude des êtres humains à prendre en considération tous les éléments qui sont d'importance semblable (Lindsay et Norman, 1980). Elle peut également être caractérisée comme étant la capacité de l'être humain à maintenir son attention sur une période de temps assez longue (Lieury, 2005). Selon Sternberg (2007), l'attention possède quatre différentes fonctions. Premièrement, elle a une fonction de détection de signal. La seconde fonction est la vigilance et l'exploration, qui ont pour but de repérer les informations pertinentes. Troisièmement, l'attention détient une fonction sélective, qui tient compte de certaines informations et qui en rejette d'autres. Pour terminer, l'attention tient une fonction partagée, c'est-à-dire qu'elle peut répartir de la bonne façon l'attention pour que deux ou plusieurs tâches puissent être réalisées en même temps.

Si la personne qui clavarde est attentive à la façon dont elle écrit ses mots et ses phrases, il est possible qu'elle fasse un nombre moindre d'erreurs relatives au français conventionnel lors des séances de clavardage, si c'est ce qu'elle désire, bien entendu. Il est important de préciser que les erreurs associées au français conventionnel, mais qui correspondent aux normes de l'écriture de la langue codée, ne constituent pas des erreurs en soi pour la communauté virtuelle. En fait, il s'agit tout simplement de l'utilisation de la langue codée. En situation de clavardage, la personne ne désire pas, sciemment, en faire une activité cognitive soutenue, donc mobiliser pleinement son attention. Il est nécessaire d'écrire rapidement, ce qui est requis par l'instantanéité des réponses et la rapidité des échanges. De plus, il est possible que si elle réalise deux activités à la fois ou plus et est alors moins attentive à ce qu'elle écrit, elle utilisera surtout la langue codée, puisque son attention sera divisée. La qualité de l'écriture ne sera donc pas priorisée. Effectivement, la personne qui clavarde avec cinq ou six personnes à la fois ne peut pas être attentive de manière égale à chaque conversation. Elle n'aura pas d'autre choix que de prioriser l'une des conversations, où elle écrira sans doute davantage, ce qui fait en sorte que les autres conversations seront mises de côté. Conséquemment, il est prévisible que la personne fasse plus de fautes de français conventionnel ou utilise davantage la langue codée. D'ailleurs, la 
langue codée semble répondre à ce besoin particulier du clavardage qui consiste à écrire simultanément plusieurs productions, ce qui n'existe pratiquement pas en contexte d'écriture formel. En effet, il est rare qu'un élève doive écrire simultanément deux textes à la fois en contexte scolaire. Habituellement, l'utilisation du français conventionnel se fait dans un contexte qui ne requiert pas un partage de l'attention sur plusieurs tâches, ce qui faciliterait son utilisation dans ces circonstances. La langue codée, de par sa flexibilité et sa polyvalence, semble répondre particulièrement bien aux besoins de partage de l'attention en contexte de clavardage.

\subsection{TRANSFERT DES APPRENTISSAGES}

Dans le cadre de l'actuelle recherche, les particularités du transfert des apprentissages doivent être soulignées. Sousa (2002) précise que le transfert des apprentissages se déroule selon deux parties, soit l'influence d'un apprentissage antérieur sur un apprentissage nouveau, ainsi que l'utilité du nouvel apprentissage dans le futur de l'apprenant. Il dénote qu'il existe un transfert positif, où un ancien apprentissage contribue positivement à un apprentissage nouveau, de même qu'un transfert négatif, lorsque les apprentissages passés font obstacle aux nouveaux. Cela entraîne de la confusion et des erreurs. Gagné (1970) a également identifié différentes sortes de transfert. En premier, le transfert informé cherche à trouver des relations de similitude entre les événements racontés en classe et le problème que l'élève doit résoudre. Si les élèves ne connaissent pas les relations possibles entre ces événements et la tâche, il s'agit alors d'un transfert que l'on peut qualifier de spontané. De

plus, le transfert vertical concerne les connaissances antérieures, qui aident directement à l'acquisition d'un nouvel apprentissage, alors que le transfert horizontal est lié au fait que les connaissances sont utilisées dans le but de résoudre une situation-problème dans un contexte différent de celui où l'apprentissage a eu lieu.

Sousa (2002) explique qu'à long terme, les éléments explicatifs du transfert des apprentissages font en sorte que l'élève devrait utiliser ses connaissances et ses habiletés 
scolaires dans les autres contextes. Par conséquent, l'élève devrait recourir à un français convenable lorsqu'il clavarde, ce qui n'est pas le cas. Il est aussi nécessaire de considérer le transfert négatif en lien avec le clavardage. Comme le précise Sousa, il est possible que les nouveaux apprentissages interfèrent avec les anciens, occasionnant de la confusion et un plus grand nombre d'erreurs. Ainsi, il est possible que l'apprentissage de la langue codée interfère avec le français conventionnel, amène de la confusion dans l'esprit des élèves et un plus grand nombre d'erreurs dans leurs écrits scolaires. Il s'agirait donc d'un cas de transfert négatif.

Par ailleurs, les différences individuelles des individus font en sorte que certains élèves ont plus de difficulté à transférer les savoirs qu'ils ont appris dans un contexte défini (Perraudeau, 1996). Par exemple, un élève en difficulté d'apprentissage peut apprendre une formule mathématique, mais ne pas être capable de l'appliquer par la suite dans le cadre d'une résolution de problème.

Il est ici nécessaire de faire un parallèle entre le transfert des apprentissages et l'objet de la présente recherche. Il est facilement observable que les jeunes ne transfèrent généralement pas un français correct, tel qu'ils l'ont appris à l'école, dans leur écriture lorsqu'ils clavardent. Ils ne font donc pas appel au français conventionnel en contexte de clavardage. Nous pouvons présumer à cet effet qu'il ne s'agit pas véritablement d'une erreur des élèves, étant donné que ce sont des erreurs intentionnelles, c'est-à-dire qu'ils savent pertinemment qu'ils n'écrivent pas de la bonne façon selon les normes de la langue française. Les jeunes sont donc conscients qu'il s'agit de deux formes de français différentes qui s'utilisent selon les contextes: en situation de clavardage et en contexte scolaire. En ce sens, il n'est pas nécessaire pour eux de transférer leurs apprentissages scolaires dans leurs écrits informatisés. Nous pouvons alors supposer que les élèves agiront de la même façon et ne transféreront pas leurs apprentissages virtuels vers leurs écrits scolaires. À cet égard, la métacognition a un rôle à jouer, étant donné que nous pouvons présumer que les élèves ayant des performances scolaires élevées ne transféreront pas le français conventionnel dans leurs écrits lorsqu'ils clavardent, car ils savent que dans ce 
contexte il n'est pas requis. Ainsi, ils font des fautes de français consciemment en clavardant. L'absence de transfert peut alors être considérée comme une action volontaire, ce qui laisse entrevoir que la situation inverse pourrait être probable, c'est-à-dire que les élèves qui clavardent ne transféreraient pas la langue codée dans leurs écrits scolaires.

Par contre, le temps d'exercice doit aussi être pris en considération. Effectivement, tel que vu précédemment, le temps de pratique a une influence sur les apprentissages, l'emmagasinage et l'automatisation des connaissances. L'élève qui passe plus de temps à clavarder en utilisant une langue codée qu'à écrire des textes où il doit employer un français conventionnel finira peut-être par la transférer dans ses écrits scolaires, étant donné qu'il a davantage travaillé cette forme d'écriture et que celle-ci, de ce fait, serait automatisée.

Afin de diminuer les risques de transfert de la langue codée dans les écrits scolaires, il serait nécessaire pour l'enseignant de favoriser l'écriture en classe pour que le temps de clavardage ne dépasse pas le temps d'écriture nécessitant un français conventionnel. Il est aussi important que les enseignants informent leurs élèves que différentes formes d'écriture coexistent et qu'il faut être capable de les différencier en tout temps. Il faut donc qu'ils expliquent aux jeunes que ce qu'ils écrivent devant leur ordinateur et à l'école est et devrait être selon deux formules différentes.

En somme, les explications concernant le transfert des apprentissages permettent de mettre en lumière que les élèves ayant de bonnes habiletés métacognitives devraient être capables de ne pas transférer la langue codée du clavardage dans leurs écrits scolaires. Toutefois, l'interrogation se situe particulièrement envers les élèves en difficulté d'apprentissage. Effectivement, ces élèves auraient des capacités métacognitives plus faibles. De plus, ils auraient aussi de la difficulté à automatiser le français conventionnel. Cela pourrait les amener à avoir plus d'affinités avec la langue codée, de par sa facilité et sa flexibilité. De ce fait, ils seraient plus susceptibles de la pratiquer et pourraient l'automatiser davantage que la langue écrite conventionnelle. Ces éléments pourraient 
représenter un risque que les élèves en difficulté d'apprentissage fassent un transfert négatif de la langue codée employée lors de sessions de clavardage vers les écrits formels.

\subsection{VARIATIONS LINGUISTIQUES}

Il est particulièrement intéressant de s'attarder aux variations linguistiques dans le contexte actuel. En effet, la langue codée employée en contexte de clavardage consiste en une langue qui a été modifiée par les usagers, de sorte qu'elle a subi différentes variations linguistiques. En ce sens, il est pertinent de s'intéresser aux explications de Gadet (2003), qui met en évidence la notion de sociolinguistique. Gadet affirme : "La sociolinguistique s'intéresse surtout à la langue parlée, porteuse de diversité, en face d'une langue écrite relativement stabilisée car ayant fait l'objet d'une standardisation »(Gadet, 2003 : 91). Le chercheur met principalement l'accent sur les variations linguistiques associées à la langue orale, qui ont été modifiées au fil des ans et où les variétés lui correspondant sont lexicales, discursives, pragmatiques, morphologiques et phonétiques. Les variations peuvent être observées autant lors de l'usage de la langue écrite qu'orale. De plus, Gadet a répertorié différents types de variations linguistiques. En premier lieu, la langue peut varier selon les usages. À cet égard, la langue employée par l'usager diffère selon la personne à qui elle s'adresse. Par exemple, la variation diachronique fait en sorte que le français utilisé est distinct selon l'histoire et les conditions sociales de la personne. Ainsi, le français d'aujourd'hui se distingue en de nombreux points de celui employé couramment il y a une centaine d'années. En second lieu, nous pouvons relever une variation diatopique (ou spatiale). Dans ce cas, nous pouvons comparer le français parlé par une personne native de Montréal et celui employé par une personne issue de certaines villes de la Gaspésie possédant un accent typique de cette région du Québec. Aussi, la variation peut être de nature diastratique (ou sociale et démographique). Ici, il est possible d'observer que les manières de parler sont dissemblables selon l'âge des interlocuteurs, le milieu socioéconomique, l'éducation... Par exemple, des personnes âgées discutant entre elles n'utiliseront pas la même langue orale qu'un groupe d'adolescents. Mis à part les variations 
linguistiques abordées précédemment dont l'usage varie selon les usagers, d'autres variations sont également à considérer. La variation diaphasique (ou individuelle), que l'on peut également qualifier de situationnelle ou stylistique, fait en sorte que la langue orale varie selon l'activité en cours, les personnes avec qui l'individu discute et le moment de la journée.

D’ailleurs, diverses manifestations langagières sont associées au phénomène de variation linguistique. Premièrement, la variation phonique se démarque par le fait que son système vocalique est particulièrement variable et que les changements assujettis à la langue par son intermédiaire sont considérables. Deuxièmement, la variation morphologique est à prendre en compte étant donné que " Les phénomènes morphologiques occupent une place de choix dans la variation, mais les variantes portent un tel poids de jugement social lorsqu'elles s'écartent de la norme qu'elles sont la plupart du temps objet de rejet et de stigmate, d'où la notion de « faute » [...]» (Gadet, 2003 : 99). Les variations syntaxiques et lexicales sont aussi à prendre en considération. Gadet mentionne à cet égard que pour la majorité des usagers, le lexique consiste en la capacité de dire des choses de différentes façons. De plus, les variations lexicales peuvent être liées aux classes sociales et différer selon l'âge des individus; Gadet explique même que des différences langagières peuvent être remarquées entre les garçons et les filles. De plus, le chercheur affirme que les modifications majeures liées au phénomène de la variation linguistique concernent principalement le lexique et la syntaxe. Concernant le lexique, le chercheur donne pour exemple l'appellation du mot voiture, que certains utilisateurs vont nommer bagnole. Il s'agit de la même chose pour le mot argent, où les usagers vont recourir au mot fric. Au plan syntaxique, les variations sont surtout visibles au niveau de l'interrogation, de la négation....

Voici quelques exemples de variations linguistiques observées dans la langue orale française. Nous pouvons caractériser ces formes de langues comme étant de « nouvelles langues ». Ainsi, l'usage de la langue intitulée loucherben consiste à enlever la première 
consonne du mot, à mettre la lettre $l$ à la place et à placer cette consonne à la fin du mot. Un suffixe peut être ajouté, tout dépendamment du désir de l'usager (exemple : louf pour où). Le javanais est également populaire par moments chez les adolescents. Cette langue consiste à insérer le groupe de mots $a v$, cela dans toutes les syllabes du mot, spécifiquement entre la consonne et la voyelle. Gadet donne pour exemple la transformation du mot citron en savitravon. Quant au verlan, celui-ci vise l'inversion de syllabes et est attesté depuis le début de $19^{\mathrm{e}}$ siècle. Toutefois, il a pris de l'ampleur au début des années 1970. Depuis ces années, il agit directement sur l'identité des adolescents issus d'un milieu défavorisé. Voici ce que mentionne le chercheur concernant les règles du verlan :

Les règles du verlan sont prévisibles pour les mots de deux syllabes, mais souvent complexes pour les autres (reup pour « père », retsiga ou garetsi pour « cigarette». À la manipulation formelle s'ajoute parfois un effet sémantique : céfran, verlan de " français », signifie « français de souche, ni beur ni black », signification pour laquelle la langue commune n'a pas de mot unique; de même pour beur, de " arabe », et de son dérivé beurette (enfant d'Arabes, nés en France et ayant la nationalité française (Gadet, $2003: 102$ ).

Le verlan fait également référence à une identification de groupe et au plaisir de jouer avec la langue à sa convenance. Des individus ont majoritairement tendance à recourir uniquement à des termes vernalisés populaires, tandis que d'autres préfèrent être créatifs dans leur utilisation du verlan et jouer davantage avec la langue. D'ailleurs, le chercheur précise que le verlan témoigne de peu de caractéristiques linguistiques particulières, mis à part aux plans lexical et phonique. L'orthographe du verlan est inconstante, notamment parce que le verlan est une langue codée où les éléments morphologiques du français ne sont pas nécessairement honorés. De plus, il demeure que le verlan est une langue qui change rapidement et dont des variations sont visibles d'un endroit à un autre. Gadet soulève un point intéressant: «On n'a pas, pour le moment, assez de recul quant à son avenir (mais il semble déjà en perte de vitesse). Une autre question est de savoir comment parleront les verlanisateurs une fois devenus adultes et mieux intégrés dans la société » (Gadet, $2003: 102$ ). Le chercheur semble donc inquiet des possibles répercussions du verlan sur la langue orale des jeunes. Le verlan, tout comme la langue associée au 
clavardage, est une langue codée qui a été modifiée au fil de la pratique par les usagers. Il semble donc légitime de s'interroger sur l'impact de ces deux langues, l'une orale et l'autre écrite, sur la maîtrise de la langue française, et de les mettre en parallèle.

Quant à Cornalba (2009), il affirme que l'une des caractéristiques de chaque génération est qu'elles tentent toujours de se soustraire aux normes linguistiques. Le chercheur aborde lui aussi le cas du verlan, qui consiste selon lui en l'énonciation de cet affranchissement des normes de la langue française. En fait, les usagers du verlan font appel à ce code oral que l'on pourrait qualifier de «nouveau rapport à la langue » en s'amusant à décomposer et à transformer la langue française. Ils ne font pas qu'inverser des syllabes; les gens faisant usage de cette langue orale codée recourent à une méthode particulière pour déconstruire la langue. Cornalba met en évidence la notion de la quête identitaire étant donné que les usagers du verlan malmènent la langue afin de définir leur identité personnelle et collective. Goudailler (2007) mentionne lui aussi que les pratiques langagières des adolescents d'aujourd'hui contribuent à définir leur identité et que le recours à certaines langues souvent codées favorise la construction de celles-ci. Ce chercheur intitule la langue alternative employée par les jeunes pour communiquer «le français contemporain des cités ». Il explique que la nouvelle langue orale employée par les adolescents se réfère avant tout aux normes de la langue française; les jeunes se basent donc sur ces normes pour la modifier de façon déterminante. Goudailler précise aussi que l'aspect identitaire associé à la transformation de la langue orale par les jeunes se remarque principalement dans les transformations du lexique de la langue. Voici ce qu'il explique :

Se développe ainsi la volonté de transgresser la norme linguistique et de créer, par instillation de traits spécifiques provenant du niveau identitaire dans le système linguistique dominant, une diglossie, véritable manifestation langagière d'une révolte (Goudailler, 2007: 120-121).

De plus, le désir de se démarquer d'autrui contribue au fait que les usagers décident de verlaniser leur langue orale, ce qui agit directement sur leur propre identité et leur impose une volonté de transgresser davantage les normes de la langue française. 
L'utilisation massive du verlan est ainsi l'indice d'un refus de la société vécue comme oppressante. Le verlan n'est pas seulement un codage (fonction cryptique), qui permet aux exclus d'exclure ceux qui les excluent (Bourdieu, 1983), mais aussi une façon de bien marquer son identité par rapport à ceux qui sont à l'extérieur du réseau de pairs de la cité. Prendre la langue de l'Autre, la transformer de telle manière qu'elle en devient méconnaissable, la renvoyer «à l'envers », traduit le rejet de cet Autre. Les diverses formations linguistiques de type verlanesque tendent donc à montrer que les variétés langagières relevées dans les cités ont un mode de fonctionnement « en miroir » par rapport à ce que l'on constate généralement dans la langue française (Goudailler, $2007:$ 122).

Selon Lioger (2002), lorsque certains individus tentent de s'éloigner des normes de la langue française en recourant à une nouvelle langue, deux possibilités sont envisagées. Les déviations de la langue peuvent être tout simplement rejetées, ou bien, au contraire, assimilées. Dans le contexte de l'assimilation, il doit alors être possible de codifier la langue.

Hagège (2006) s'est également attardé au phénomène. Ce dernier souligne que de nombreux usages oraux cohabitent avec les normes de la langue française. Le chercheur mentionne par rapport au respect des normes de la langue et son usage parfois déviant que tous, incluant les professionnels en linguistique et les écrivains, et même ceux qui s'opposent au recours à une norme stricte, déclarent que l'école a le devoir d'enseigner le français selon ses normes; il s'agit d'attentes exprimées autant par les élèves que par leurs familles.

De l'avis de Mercier (2002), le fait que la langue subisse des variations linguistiques n'est pas le signe d'un fonctionnement déficient, mais bien d'une adaptabilité, d'une flexibilité et d'une souplesse importantes. La langue française s'adapte donc en fonction des interlocuteurs y faisant appel. Les individus recourant à une langue, consciemment ou non, choisissent dans leur répertoire de mots ceux qui leur semblent les plus adéquats pour énoncer leur message le plus clairement possible. Ils peuvent choisir d'utiliser un mot en particulier puisqu'ils sont habitués à recourir à ce mot, ou chercher à rompre l'habitude qu'ils ont à l'employer en cherchant à adapter la langue qu'ils utilisent à l'accoutumée. De 
plus, les individus ne possèdent pas les mêmes connaissances par rapport à la langue compte tenu que leur âge, leurs expériences de vie, leur éducation et bien autres éléments qui ont une influence directe sur leur connaissance de la langue (Mercier, 2002). Lorsque les jeunes clavardent, ils choisissent dans leur répertoire de mots ceux qu'ils désirent employer. Pour ce faire, ils adaptent à langue française et font montre de diverses variations linguistiques.

Somme toute, il semble de circonstance de considérer la langue codée associée au clavardage comme étant une sorte de variation linguistique. Effectivement, les jeunes d'aujourd'hui s'amusent à transgresser les normes de la langue française écrite et à utiliser leur propre code reflétant leur identité. D'un autre côté, plusieurs similitudes ont été remarquées entre le verlan et la langue codée liée au clavardage, compte tenu que bien que l'une soit écrite et l'autre orale, ces langues ont été modifiées de façon importante au gré des envies des usagers et souvent, d'un groupe social en particulier, soit les adolescents. Lorsque les jeunes sont en situation de clavardage, ils s'amusent à modifier leur langue à leur gré.

\subsection{LE RECOURS AU VERLAN ET LA MAITRISE DE LA LANGUE FRANÇAISE ORALE}

Selon Bertucci (2003), la langue orale privilégiée par les jeunes français d'aujourd'hui est le verlan et est particulièrement utilisée en France, autant en milieu rural qu'en milieu urbain, à l'école qu'à l'extérieur de celle-ci. Ils y recourent de façon machinale. La recherche d'identité et d'association à un groupe d'appartenance contribue au fait que les élèves tiennent à utiliser cette forme de langue qui ne correspond pas du tout au français parlé conventionnel et où la grossièreté et l'obscénité sont de mise. Les élèves questionnés dans le cadre de l'étude de la chercheuse mentionnent qu'ils savent quand parler verlan et quand ils doivent utiliser un français correct, surtout à l'école. Ils jugent donc qu'il n'est pas difficile de parler à la fois le français et le verlan. Ces jeunes sont d'avis que recourir à cette langue est nécessaire s'ils désirent faire partie d'un groupe et être 
intégrés en milieu scolaire. Bertucci souligne même que la façon dont le verlan est mis en œuvre peut être considérée comme une performance. De plus, elle insiste sur le fait que plus les jeunes utilisent le verlan pour communiquer, plus ils sont généralement déviants aux niveaux social et scolaire.

Elle fait également le lien entre les élèves forts et les élèves en difficulté d'apprentissage. Effectivement, elle mentionne que ceux ayant de grandes lacunes en français perçoivent moins qu'il s'agit d'une langue qui ne doit pas être utilisée dans d'autres contextes, ce qui est particulièrement handicapant pour cette clientèle de jeunes. Il suffit de cette constatation pour que ces élèves soient en situation d'échec, tout simplement parce qu'ils recourent à une langue orale qui ne correspond pas du tout aux balises d'un français convenable. Voici ce qu'indique la chercheuse concernant cette langue :

La rupture est d'autant plus forte, quand elle se produit en milieu scolaire, puisque la présence même de ces parlers au sein de l'école est un défi, une provocation au français scolaire et aux enseignants qui le transmettent, dans un lieu qui n'est plus sanctuarisé et protégé par sa seule existence en tant qu'institution (Bertucci, 2003 : 26).

Il est donc légitime de se questionner concernant les élèves en difficulté et le clavardage. Si ces jeunes ont peine à différencier les contextes d'utilisation du verlan et du français oral conventionnel, cela pourrait être également le cas pour ce qui est du clavardage. Ainsi, ces élèves pourraient mal distinguer les contextes requérant la langue codée ou le français conventionnel.

\subsection{1 ÉCRITURE ET STRATEGIES D'ECRITURE}

Expliquons maintenant l'écriture en tant que telle, puisqu'elle demeure un élément clé de ce mémoire. Préfontaine et Lebrun (1992) abordent l'écriture d'un point de vue créateur de façon très intéressante. Il est possible de lier certains points de cette citation à la présente recherche, puisque ces caractéristiques de l'écriture peuvent pour plusieurs être attribuées au clavardage : 
[...] pour écrire, il faut apprendre à regarder, à observer, à rassembler, à classer, à situer, à analyser, à couper, à ajouter, à préciser, à décrire, à s'émerveiller, à s'intérioriser pour extérioriser, à percevoir des globalités, à les détailler, à les agencer en séquences logiques ou chronologiques, à en hiérarchiser les divers constituants, à séduire, à charmer, à provoquer et à émerveiller, à laisser en attente, à susciter l'intérêt, à retenir l'attention; écrire, c'est percevoir un tout et le rendre disponible aux autres dans tous ses aspects, leur en lire les diverses facettes. Écrire, c'est créer un monde, son monde, que l'on donne aux autres; c'est les en enrichir : écrire, c'est s'apporter aux autres (Préfontaine et Lebrun, $1992: 33$ ).

Lorsque l'apprenant rédige un texte, il doit sélectionner les éléments nécessaires et planifier la façon dont les informations seront organisées dans son texte. Écrire peut également être défini comme une textualisation séquentielle et définitive d'une idée qui a été en premier organisée au niveau mental. L'élève exprime une idée au fur et à mesure et prend le temps de la préciser davantage. Par contre, il doit accorder les verbes et les noms, réfléchir aux mots adéquats qui n'altéreront pas le sens de sa phrase, penser à l'orthographe des mots... Lors des cours de français, l'enseignant apprend aux élèves les normes de la langue française ainsi que tous les éléments applicables dans un contexte de lecture et d'écriture. En revanche, pour eux, il est parfois difficile de faire le pont entre la théorie et la pratique concernant les aspects formels de l'écriture. C'est pourquoi des erreurs sont visibles dans des textes d'enfants, d'adolescents, et même d'adultes ayant terminé leurs études depuis plusieurs années et possédant malgré tout une bonne maitrise de la langue (Préfontaine et Lebrun, 1992).

Dans le cadre du processus d'écriture, des stratégies cognitives sont requises. Boudreau (1995) s'est attardé aux stratégies cognitives utilisées lors de la rédaction d'un texte en contexte scolaire. Les processus cognitifs requis lors de la construction d'un texte sont les suivants : la planification, la rédaction et la révision.

Durant la période de planification, l'élève donne un support à ce qu'il désire écrire; il planifie alors son texte. La seconde période consiste en la rédaction du texte. Le dernier processus est la révision, où les élèves doivent corriger leurs erreurs dans le but d'améliorer leur texte (Boudreau, 1995). 
Boudreau explique que les élèves disposant de bonnes stratégies d'écriture ont dû au départ atteindre un certain automatisme concernant l'orthographe; ils sont donc habitués à écrire de façon convenable. Ils n'ont pas besoin de s'attarder aux habiletés motrices, aux supports dans le but de maintenir une certaine pertinence dans le texte, aux mécanismes de rédaction, ainsi que constamment réfléchir aux diverses stratégies utilisées. Ceux ayant des difficultés ne penseront pas à ces éléments de façon naturelle; ils devront y réfléchir durant le processus d'écriture, ce qui fait en sorte qu'ils auront moins de temps, ou d'unités de traitement de l'information disponibles, pour planifier, rédiger et réviser leur production (Boudreau, 1995). De plus, il est possible que plusieurs unités de travail soient remplies seulement avec les éléments mentionnés précédemment ; ces élèves auront donc moins de place pour traiter les autres aspects liés à la tâche d'écriture. En revanche, il est important de préciser que ces stratégies sont requises principalement en classe. Selon les erreurs facilement visibles sur les sites de clavardage, les jeunes ne prennent sans doute pas le temps d'y recourir en clavardant, ce qui soulève un questionnement. Effectivement, par automatisation due à la pratique du clavardage et par le transfert négatif, il est possible qu'ils omettent l'utilisation des stratégies d'écriture liées au processus rédactionnel en contexte d'écriture formel.

À vrai dire, si ces élèves plus faibles au niveau académique ont de la difficulté au départ à automatiser ces stratégies d'écriture, comment s'y prendront-ils pour écrire un texte en milieu scolaire s'ils sont pris entretemps dans l'engrenage du clavardage et qu'ils en font une activité quotidienne? Tous ces éléments font en sorte qu'il est plausible qu'il soit plus difficile pour ces élèves de performer en contexte formel d'écriture. Ces éléments semblent donc démontrer que le clavardage n'interfère pas seulement avec l'orthographe et la syntaxe, mais également avec les stratégies d'écriture liées au processus rédactionnel, soit la planification, la rédaction et la révision. En effet, nous savons que les élèves ayant recours au clavardage ne planifient pas et ne révisent pas leur texte en situation de clavardage. Au fil du temps, il serait possible que cela soit généralisable aux écrits conventionnels. 


\subsection{Conclusion}

Ce cadre théorique avait pour objectif d'utiliser la psychologie cognitive pour expliquer le phénomène auquel est consacré ce mémoire de maîtrise, soit l'influence du clavardage sur la maîtrise du français écrit des élèves du premier cycle du secondaire. Certaines explications issues de ce cadre théorique semblent démontrer que le français conventionnel ne doit pas craindre l'avènement du clavardage, tandis que d'autres vont dans le sens inverse, c'est-à-dire qu'elles prédestinent que l'essor du clavardage doit être considéré avec une certaine inquiétude, surtout chez les élèves en difficulté.

D'entrée de jeu, il importe d'expliquer que le concept à la base du cadre théorique est la métacognition. Cet élément constitue une caractéristique de la psychologie cognitive et favorise la compréhension du sujet de la présente étude. La métacognition a été analysée selon deux points de vue. L'un d'entre eux est favorable au clavardage, alors que le second laisse entendre que les méfaits du clavardage doivent être davantage pris en compte.

Abordons la réalité des élèves en situation de réussite. Notamment, ces élèves possèdent de hautes aptitudes métacognitives et devraient, selon les écrits de la psychologie cognitive, être capables de distinguer à quelle forme d'écriture ils doivent recourir selon les contextes, soit le français conventionnel lorsqu'ils écrivent un texte en classe et la langue codée lorsqu'ils clavardent. Puisque l'expérimentation se déroule avec des groupes réguliers, il est prévisible que la majorité de ces élèves possèdent de bonnes aptitudes et stratégies métacognitives, ce qui fait en sorte qu'ils devraient faire la part des choses et ne pas transférer des mots codés du clavardage dans leurs écrits scolaires. De l'avis de Grangeat (1997), un élève est en situation de réussite académique s'il est capable de recourir à ses connaissances et à les appliquer dans diverses situations. Nous pouvons lier cette affirmation aux connaissances conditionnelles, qui concernent justement les conditions d'application des connaissances.

Pour ce qui est du système cognitif en tant que tel, il importe de préciser que si l'élève est à l'écoute de son fonctionnement cognitif lorsqu'il réalise une tâche lui 
demandant une certaine réflexion, il ne devrait pas avoir de difficulté à reconnaître quand il doit utiliser chaque forme d'écriture (écriture conventionnelle et langue codée).

En revanche, au fil des ans, les adolescents ont appris une gamme de mots écrits, ce qui fait en sorte qu'ils ont développé un bagage orthographique riche et varié. Malgré le fait qu'ils ont ces mots en mémoire, les jeunes d'aujourd'hui choisissent volontairement de ne pas les employer à chaque fois qu'ils ont recours à ce mode de communication qu'est le clavardage. Cela est dû au fait que la langue reliée au clavardage constitue une langue alternative, qui ne doit pas être employée dans toutes les situations. Ainsi, lorsqu'ils ont débuté à clavarder, ils ont fait l'apprentissage d'un nouveau répertoire de mots, qu'ils ont également fini par mémoriser au fil du temps. En fait, lorsque les adolescents clavardent, ils savent automatiquement de quelle façon ils doivent écrire pour suivre les normes correspondant à la communauté virtuelle. Qui plus est, plus ils passent de temps à clavarder, plus ils automatisent la langue associée au clavardage puisqu'ils la mettent constamment en pratique, ce qui favorise par le fait même la mémorisation de cette langue. Ils apprennent donc la langue codée de cette manière. Par exemple, plus ils font appel à un mot, comme pourquoi qu'ils écriront pkoi, plus la façon d'écrire ce mot selon les caractéristiques de la langue codée est ancrée dans leur mémoire et y demeure. Il est plausible de se demander si, avec le temps, ces jeunes finiront par ne plus distinguer quelles lettres ils doivent utiliser pour le même groupe de mots ou le même mot, ce qui pourrait engendrer des erreurs dans leurs écrits scolaires. Il est tout de même possible que ces erreurs soient visibles à long terme seulement, compte tenu qu'ils prendront forcément du temps avant d'automatiser ces expressions.

Toutefois, nous pouvons aussi penser que ces jeunes possèdent dans leur répertoire cognitif deux différentes mémorisations du même mot qu'ils emploient selon le contexte, soit en classe et en situation de clavardage. Dans ce cas, ils seraient aptes à distinguer les deux formes de français, sans que l'une n'interfère avec l'autre.

Dans un autre ordre d'idées, le transfert des apprentissages en lien avec l'objet de cette recherche se doit d'être abordé. Nous savons que les jeunes ne transfèrent pas le 
français conventionnel dans leurs écrits lorsqu'ils clavardent avec leurs pairs, pour la raison qu'ils s'adaptent aux normes virtuelles, mis à part pour certains mots, bien entendu. En revanche, ils sont conscients que leur écriture codée comporte des erreurs, particulièrement si cette écriture est comparée avec un français respectant les normes de la langue française. Ils agissent donc de façon volontaire puisqu'ils savent qu'ils n'écrivent pas de façon adéquate si l'on se fie aux normes de la langue. Ainsi, il est loisible de penser que s'ils sont capables de ne pas transférer ces écrits, ils seront capables d'agir de la même manière lors de la situation inverse, c'est-à-dire qu'ils ne transféreront pas les écrits du clavardage dans leurs écrits formels à l'école. Il est évident que la métacognition a son rôle à jouer en ce qui concerne le transfert des apprentissages. Effectivement, un élève ayant de bonnes aptitudes métacognitives devrait être susceptible d'agir tel que le supposent les explications mentionnées précédemment, de faire la part des choses et de ne pas recourir aux mots du clavardage lors d'une rédaction.

Les variations linguistiques doivent aussi être prises en compte. Gadet (2003) s'est notamment intéressé à la question. Les langues peuvent subir différentes modifications, qu'elles soient lexicales, pragmatiques, discursives, morphologiques ou phonétiques. Les individus peuvent effectuer des changements à la langue orale et écrite tout dépendamment de la personne avec qui ils sont en communication, du contexte, de l'éducation, du milieu, de l'âge...Plusieurs chercheurs (Gadet, 2003; Goudailler, 2007; Cornalba, 2009) abordent notamment le cas du verlan. Si ce n'est qu'il s'agit d'une langue orale, le verlan possède plusieurs similitudes avec la langue codée employée couramment en contexte de clavardage étant donné que les deux langues emploient un code qui transgresse les normes de la langue française. Bertucci (2003) mentionne elle aussi qu'il est possible de faire plusieurs liens entre la langue exploitée lors du clavardage et la langue orale (verlan) utilisée par les adolescents français. Il s'agit en effet de deux variations linguistiques de la langue française, l'une écrite et l'autre orale. La chercheuse explique que les jeunes n'utilisent pas cette langue lorsqu'ils discutent en contexte scolaire. Ils sont capables d'établir qu'ils ne doivent pas parler le verlan à l'école, particulièrement avec leurs enseignants. Ils sont ainsi aptes à faire la part des choses et à reconnaître qu'ils ne doivent pas utiliser cette langue en 
milieu scolaire. Il est donc facile de se demander si le constat sera le même par rapport au clavardage, c'est-à-dire que les jeunes seraient capables de distinguer quand ils peuvent utiliser la langue codée du clavardage de quand ils ne peuvent pas le faire. Par contre, selon l'auteure, il pourrait en être autrement pour les élèves en difficulté. Celle-ci est d'avis que les jeunes ayant des difficultés d'apprentissage en français pourraient moins percevoir qu'il s'agit d'une langue orale (verlan) qui ne doit pas être utilisée partout.

Malgré certains éléments de la psychologique cognitive qui tendent vers le fait que le clavardage n’interférerait pas sur la maîtrise de la langue française écrite, d'autres laissent entendre que cela pourrait bien être le cas. Notamment, pour revenir à la métacognition, le clavardage ne devrait pas affecter la maîtrise du français écrit des élèves du premier cycle du secondaire tel que mentionné précédemment. Il est toutefois légitime de s'interroger pour ce qui est des jeunes en difficulté d'apprentissage, surtout que, selon Tardif (1992), la métacognition permet de distinguer les jeunes en difficulté des jeunes qui sont plus forts académiquement, ce qui démontre qu'il s'agit de deux groupes bien distincts. Au Québec, les élèves en difficulté d'apprentissage sont souvent intégrés dans les classes régulières. Cela fait en sorte que leur cas est également analysé globalement par l'intermédiaire de ce mémoire. Il est possible que les élèves vivant avec des difficultés, qui ont pour plusieurs au départ des lacunes aux niveaux de la verbalisation des tâches effectuées et de l'autoévaluation de leurs performances scolaires, subissent davantage les effets négatifs possibles du clavardage par rapport à la langue. En outre, d'après Bazin et Girerd (1997), les difficultés des élèves peuvent être dues à une déficience de niveau métacognitif, ce qui fait en sorte que ces élèves ont peine à réussir comme ils le souhaiteraient. Nous pouvons donc supposer que la métacognition a un rôle à jouer en ce qui concerne la capacité de l'élève de distinguer les différents contextes d'écriture et de transférer ou non la langue codée du clavardage dans leurs écrits scolaires. Ainsi, les élèves en difficulté pourraient être plus à risque de transférer les mots codés du clavardage dans leurs écrits scolaires, où ils doivent employer un français conventionnel. 
Par ailleurs, il est possible que les élèves en difficulté ne maîtrisent pas de façon suffisante les connaissances déclaratives, procédurales et conditionnelles nécessaires à la rédaction d'un texte de qualité en contexte scolaire. Il est probable qu'ils apprécient et utilisent davantage l'écriture requise en contexte de clavardage, étant donné qu'elle fait appel à une langue codée qui se démarque par le fait que les utilisateurs s'amusent à changer les normes de la langue française. De surcroît, peut-être que les jeunes plus faibles, qui ont déjà de la difficulté à maîtriser les connaissances déclaratives et procédurales concernant la langue française conventionnelle, automatiseraient une langue incorrecte; d'ailleurs, leur faible métacognition pourrait amener un transfert négatif de la langue codée dans les écrits formels requis en contexte scolaire.

Il est aussi nécessaire de prendre en compte la répétition à cet égard. En effet, l'élève qui clavarde énormément, par exemple 20 heures hebdomadaires, comparativement à celui qui clavarde deux heures par semaine seulement, pourrait peut-être davantage automatiser la langue codée reliée au clavardage, étant donné qu'il l'a davantage travaillée que le français conventionnel. Ainsi, il est possible que les élèves qui clavardent beaucoup soient plus susceptibles de subir les effets néfastes du clavardage à long terme.

En outre, nous savons que les élèves recourent majoritairement à des stratégies d'écriture lorsqu'ils sont en processus rédactionnel dans un contexte scolaire. Il s'agit des stratégies de planification, de rédaction et de la révision de leur production écrite (Boudreau, 1995). Pour les élèves en situation de réussite, soit ceux qui disposent de bonnes stratégies d'écriture, ceux-ci ont au fil du temps développé un certain automatisme qui fait en sorte qu'ils savent quelles actions entreprendre pour rédiger un texte convenable. Ainsi, il n’est pas nécessaire pour eux de réfléchir constamment à des éléments tels que les mécanismes de rédaction et les stratégies auxquelles ils doivent faire appel. En revanche, il en est autrement pour les élèves en difficulté d'apprentissage. Ces mêmes élèves, comparativement aux élèves plus forts, ne réfléchissent pas spontanément aux éléments qui pourraient les aider à bien performer. Cela contribue au fait qu'ils prennent plus de temps à traiter ces stratégies que les élèves plus forts, ce qui fait en sorte que la tâche d'écriture 
mobilise plus d'efforts. En revanche, lorsqu'ils clavardent, les élèves, qu'ils soient en difficulté ou non, n'exploitent pas de stratégies d'écriture. L'omission de ces stratégies peut-elle finir elle aussi par s'automatiser, de sorte que les élèves ne prendraient plus le temps d'en faire usage en contexte d'écriture formel? De même, il importe de préciser que les élèves en difficulté pourraient être plus à risque à cet égard.

Ce qui nous mène à la question de recherche à la base de ce mémoire de maîtrise : quelle est l'influence du clavardage sur la maîtrise du français écrit des élèves du premier cycle du secondaire?

Selon les écrits recensés dans le cadre théorique, nous devons prendre en considération que les jeunes ont tendance à automatiser leurs apprentissages au fil de la pratique, ce qui pourrait bien être également le cas avec la langue codée utilisée en contexte de clavardage. De plus, les élèves en difficulté d'apprentissage, qui sont souvent intégrés dans des classes régulières, sont particulièrement à risque de transférer la langue codée du clavardage dans leurs écrits formels. Effectivement, s'ils sont plus enclins à clavarder qu'à écrire en contexte formel, ce qui est dû à l'accessibilité de la langue codée qu'ils automatiseraient de cette façon, et en présence de plus faibles capacités cognitives et métacognitives, peut-être seront-ils plus susceptibles de ne pas distinguer les divers contextes d'écriture. En somme, cela pourrait les amener à faire un transfert négatif de la langue codée dans leurs écrits scolaires. Suite à ces constats, nous émettons l'hypothèse que le clavardage a une influence défavorable sur la maîtrise du français écrit des élèves du premier cycle du secondaire. Ainsi, trois hypothèses, issues de cette hypothèse principale, seront vérifiées dans le cadre de cette recherche:

- Plus les élèves débutent le clavardage à un jeune âge, plus ils sont susceptibles de faire des erreurs de français associées au clavardage;

- Plus les élèves clavardent durant la semaine, plus ils sont susceptibles de faire des erreurs de français associées au clavardage; 
- Les élèves qui clavardent font davantage d'erreurs de français associées au clavardage comparativement à ceux qui ne clavardent pas. 


\section{CHAPITRE 4}

\section{MÉTHODOLOGIE}

\subsection{INTRODUCTION}

Ce chapitre explicite en détail la méthodologie liée à cette recherche, dont le but est de vérifier si le clavardage a une influence sur la maîtrise du français écrit des élèves du premier cycle du secondaire. Ce chapitre est constitué de cinq parties. Tout d'abord, l'hypothèse, la procédure, ainsi que l'échantillon seront présentés. Ensuite seront expliqués les instruments de mesure. Pour terminer, la méthode d'analyses statistiques sera détaillée.

\subsection{Procedure}

En tout premier, une rencontre avec la directrice des services éducatifs d'une commission scolaire du Bas-St-Laurent a eu lieu en août 2010 dans le but de lui présenter le projet de recherche et d'obtenir l'accord de la commission scolaire pour sa réalisation. Suite à cette rencontre, la directrice des services éducatifs a contacté la directrice adjointe de l'école préalablement ciblée dans le but de véritier son intérêt à ce qu'une telle recherche se déroule au sein de son établissement scolaire. Ensuite, cette directrice d'école, qui a accepté d'impliquer son école au projet, a elle aussi été rencontrée par l'étudiantechercheuse. Elle a ensuite communiqué avec les enseignants de français du premier cycle du secondaire de son établissement dans le but de solliciter leur participation, sans toutefois leur mettre de pression. S'ils étaient intéressés à participer à la recherche en question, ils avaient pour instructions de nous joindre ou de le mentionner à la directrice adjointe, qui 
demeurait en communication avec nous. Elle nous a fait savoir que trois enseignants se sont manifestés. Après ce premier contact, la directrice adjointe leur a remis un document informatif sur notre projet, dans le but de le leur faire connaître. Ces trois enseignants ont ainsi eu en main tous les renseignements concernant les étapes nécessaires à l'exécution du projet. Ils ont ensuite été recontactés par la directrice adjointe, qui s'est chargée de planifier un horaire dans le cadre duquel nous sommes venues présenter le projet aux élèves. Cet horaire a été élaboré selon les disponibilités des enseignants, auxquelles nous nous sommes adaptées.

La seconde étape consistait à aller en classe présenter le projet ainsi que son déroulement aux élèves. Cette étape a été réalisée 1e 28 octobre 2010 auprès de sept groupes totalisant 192 jeunes. Lors de cette première visite en classe, il leur a été expliqué que l'étude visait à déterminer quelles sont les erreurs de français écrit les plus souvent commises par les élèves correspondant à leur groupe d'âge. Ils ont eu un formulaire de consentement à signer pour signifier leur accord concernant leur participation et un second formulaire leur a été remis pour leurs parents. Ce formulaire devait absolument être signé par ces derniers afin que leur enfant puisse participer à la recherche. Sinon, les jeunes dont une des deux ou les deux signatures (signature de l'élève et des parents) étaient manquantes ont vu rejeter leur participation au projet. Les élèves et les enseignants ont été avisés que les formulaires devaient être retournés au plus tard deux semaines après sa remise, soit le 10 novembre. Lors de la présentation du projet, nous avons demandé aux enseignants une liste d'élèves, qui nous a été utile lorsqu'est venu le temps de vérifier le nombre de sujets participant à la concrétisation du projet, selon les formulaires de consentement retournés. De même, il leur a été précisé qu'ils devaient recueillir les formulaires de consentement parental eux-mêmes et les laisser à notre intention au secrétariat de leur établissement scolaire avant la date limite. Nous avons donc pu cocher sur une liste les élèves dont les formulaires ont été remis; ces élèves seulement ont réalisé la dictée et, quelques jours plus tard, rempli le questionnaire. Sur les 192 élèves ayant assisté à la présentation du projet, 169 d'entre eux ont remis les formulaires de consentement signés. De plus, 11 sujets ont été 
absents à l'une des deux étapes de la recherche; cette contrainte a fait en sorte que finalement, 158 d'entre eux ont officiellement participé à la recherche.

Étant donné que l'étude porte sur l'influence du clavardage sur la maîtrise du français écrit, il était prévisible que les élèves fassent beaucoup plus attention durant la dictée aux mots qu'ils écrivent souvent dans le cadre d'une séance de clavardage s'ils avaient été au courant du but de la recherche. Cela aurait pu biaiser les résultats, ce qui aurait eu une incidence sur leur validité. Les élèves auraient pu en effet être tentés de se corriger davantage lorsqu'ils reconnaissaient l'un de ces termes. Pour ces raisons, les participants, ainsi que leurs parents, n'ont pas été avisés de la véritable nature de l'expérimentation. Si les parents avaient été au courant, il aurait été possible qu'ils en discutent avec leurs adolescents. Une étude dite centrée sur les erreurs fréquentes en français des élèves du premier cycle du secondaire est crédible et facilement réalisable, sans toutefois préciser que les erreurs commises sur des mots reliés au clavardage seront principalement étudiées. Bien entendu, la directrice des services éducatifs de la commission scolaire, la directrice adjointe de l'école, de même que les enseignants concernés, étaient quant à eux au courant du véritable objectif de l'étude. Par contre, ils devaient s'abstenir de le préciser à leurs élèves. L’importance de cet aspect leur a été rappelée avant la présentation du projet.

D'autre part, la confidentialité des données a été préservée et les renseignements touchant aux sujets ont été classés sous clé. Un code de recherche a été associé à chaque élève, ce qui fait en sorte qu'aucun nom n'a pu être relié à une épreuve en particulier, sauf pour l'étudiante-chercheuse, qui en connaît les codes. Lors de la remise des épreuves, le nom de l'élève a été prononcé à l'oral, mais n'a pas été écrit sur le document. Le document remis pour la dictée consistait en une feuille blanche lignée, recto-verso. Une étiquette amovible où le nom de l'élève était écrit a été collée sur la feuille. Puis, lorsque l'élève a été nommé pour venir chercher sa copie, cette étiquette a été enlevée, ce qui fait en sorte que seul le code est demeuré sur la dictée. Il a donc été impossible de faire des liens entre les dictées et le nom des élèves, ce qui met en évidence le respect de la règle de confidentialité, qui est nécessaire lors de l'élaboration d'une recherche respectant les 
normes éthiques. Concernant ces normes, il importe de préciser qu'en plus de la confidentialité, les notions de consentement libre et éclairé, du respect de la dignité humaine, ainsi que du respect de la vie privée et des renseignements personnels, ont été respectées en tout temps dans le cadre de cette recherche. D'ailleurs, un certificat d'éthique a été délivré à l'étudiante-chercheuse responsable pour le déroulement de cette recherche par le comité d'éthique de la recherche de l'Université du Québec à Rimouski.

Suite à la remise des formulaires de consentement, la dictée a été effectuée dans les classes participantes selon un horaire prédéterminé avec les enseignants. Celle-ci s'est déroulée les 15, 16 et 17 novembre 2010. Son but était de mesurer si le clavardage a un impact sur la maîtrise du français écrit des utilisateurs en démontrant, entre autres, le dénombrement des mots de la dictée s'apparentant à la langue codée. Moins d'une période d'une durée d'une heure a été nécessaire à l'accomplissement de la dictée. Il a été mentionné à l'avance aux enseignants qu'il serait pertinent de prévoir du travail supplémentaire au cas où la dictée s'achèverait plus rapidement que prévu, ainsi que pour les élèves ne participant pas à l'activité.

Les sujets ont eu comme instructions de tout simplement écrire les phrases qui leur étaient dictées. Nous avons mis en évidence le fait que, lors de la correction, l'accent sera mis sur les fautes de français communes aux élèves du premier cycle du secondaire. Nous cherchons à étudier les impacts du clavardage dans une situation naturelle de classe et pour ce faire, il était nécessaire que les élèves écrivent comme ils en ont l'habitude, avec l'utilisation de leurs stratégies habituelles lors de la rédaction de textes ou la réalisation de dictées dans leur cours de français régulier.

Le questionnaire sur les habitudes informatiques a été réalisé après la dictée pour la raison que les élèves ne devaient pas connaître les véritables intentions de la dictée avant que celle-ci ne se déroule. Les questions concernant le temps passé à clavarder et l'écriture employée dans ce contexte leur auraient fourni un très bon indice. De plus, si la passation du questionnaire s'était déroulée après la dictée durant la même période, les élèves des différents groupes concernés auraient pu en discuter entre eux. C'est pourquoi nous avons 
prévu quelques jours d'intervalle entre la réalisation de la dictée et la passation du questionnaire sur les habitudes informatiques. Pour ces raisons, les enseignants ont été prévenus qu'ils pouvaient remettre le questionnaire à leurs élèves à partir du 18 novembre 2010, selon leurs disponibilités. Ils devaient communiquer avec nous par la suite pour la cueillette des questionnaires. Pour ce questionnaire, un code, qui était le même que pour la dictée, a été attribué à chaque élève, numéro que l'enseignant devait respecter lors de sa remise. Une étiquette amovible avec le nom de l'élève a été collée sur le questionnaire et l'enseignant devait la retirer tout en remettant sa copie à l'élève. Il n'est resté que le code sur le document, ce qui fait en sorte que le questionnaire a pu être lié par l'étudiantechercheuse au code indiqué sur la dictée lors de la saisie des données.

Suite à cette analyse de données, les résultats obtenus ont été communiqués en mai 2011 à la directrice des services éducatifs de la commission scolaire, à la directrice adjointe de l'école concernée et aux enseignants, par le biais d'un compte-rendu écrit. Pour ce qui est des élèves, il leur a été mentionné lors de la présentation du projet d'octobre qu'un compte-rendu oral leur serait présenté. Cette dernière étape a été réalisée les 4 et 5 mai 2011 .

\section{3 ÉCHANTILLON}

Les participants à la recherche sont des élèves entre 12 et 16 ans étudiant dans une même polyvalente du Bas-St-Laurent. L'échantillon est constitué de 158 sujets, répartis en sept groupes du premier cycle du secondaire. L'échantillon a été formé à partir de groupes existants. Il s'agit de 90 élèves de sexe masculin, ce qui totalise $57 \%$ de l'échantillon total, ainsi que 68 filles, qui représentent quant à elles $43 \%$ de l'échantillon. En somme, le groupe de sujets compte 86 élèves de secondaire un et 72 élèves de secondaire deux. Ils sont issus de groupes réguliers. Voici un tableau de compilation des sujets selon leur groupe-classe, leur niveau scolaire ainsi que leur genre. 
Tableau 5: Principales caractéristiques des sujets de l'échantillon

\begin{tabular}{|c|c|c|c|c|}
\hline Groupe & Secondaire & $\begin{array}{c}\text { Nombre de } \\
\text { garçons }\end{array}$ & $\begin{array}{c}\text { Nombre de } \\
\text { filles }\end{array}$ & $\begin{array}{c}\text { Nombre } \\
\text { d'élèves }\end{array}$ \\
\hline 1 & 1 & 17 & 4 & 21 \\
\hline 2 & 1 & 7 & 15 & 22 \\
\hline 3 & 1 & 13 & 7 & 20 \\
\hline 4 & 1 & 14 & 9 & 23 \\
\hline 5 & 2 & 17 & 6 & 23 \\
\hline 6 & 2 & 11 & 17 & 21 \\
\hline 7 & 2 & 12 & 9 & 158 \\
\hline Total & - & 90 & 68 & \\
\hline
\end{tabular}




\subsection{INSTRUMENTS DE MESURE}

Voici des explications détaillées concernant les instruments de mesure nécessaires à la réalisation de la recherche.

\subsubsection{Séances d'observations sur les sites consacrés au clavardage}

Des séances d'observations de discussions par le biais du clavardage ont d'abord été réalisées sur des sites Internet populaires québécois et français destinés aux adolescents et ayant le clavardage pour fonction. Un pseudonyme a été requis lors de ces séances d'observations. Par contre, il s'agissait simplement d'observations. Il n'y a donc eu aucune part aux discussions. Il y a plusieurs sites de clavardage où nous pouvons observer les écrits des autres internautes; il s'agit en fait de bavardoirs publics. Nous avons donc choisi de tels sites pour observer la langue codée attribuée au clavardage. Il s'agissait d'une étape préalable à l'élaboration de la dictée, puisque les mots les plus souvent changés et simplifiés que l'on a intégrés dans la dictée ont été ciblés sur ces sites. Certaines caractéristiques de l'écriture codée, qui étaient parfois flagrantes, ont ainsi pu être décelées de cette façon. Ces séances d'observations totalisent environ trois heures.

\subsubsection{Dictée}

Suite à ces séances d'observations, les mots de vocabulaire les plus couramment abrégés et modifiés durant les séances de clavardage observées ont été regroupés dans une liste, qui a ensuite été utilisée pour bâtir la dictée. Plusieurs de ces mots ont été intégrés dans la dictée afin de vérifier s'ils seront bien orthographiés en contexte scolaire. La dictée en question a été rédigée sous forme de dialogue et est d'une longueur d'une page. Le dialogue a été préconisé étant donné ses similitudes avec les discussions par l'intermédiaire du clavardage. Il s'agit d'une discussion courante entre deux adolescentes touchant à 
l'école et aux activités parascolaires. Elle compte 236 mots et figure en annexe I. Après la dictée, il a été demandé aux élèves d'écrire cinq lignes supplémentaires, donc de continuer la dictée selon leurs propres idées. Ainsi, la variable erreurs de français associées au clavardage a été mesurée par le dénombrement des mots s'apparentant à la langue codée, donc par les erreurs de français associées au clavardage qui se sont révélées dans les dictées réalisées par les sujets. Il s'agit autant des mots cités lors de la dictée que des mots ajoutés à sa suite par les élèves eux-mêmes. La variable erreurs de français associées au clavardage est donc une variable continue, étant donné que la quantité d'erreurs produites par les élèves dans leur dictée peut prendre une grande quantité de valeurs. De ce fait, elle est de nature quantitative.

Par l'intermédiaire d'un questionnaire sur les habitudes informatiques, qui constitue le second instrument de mesure et qui sera expliqué au point 4.4.3, les sujets, lorsqu'ils ont répondu à la question huit, ont dû décrire la langue utilisée lorsqu'ils clavardent avec leurs amis. Nous nous sommes entre autres basées sur ces commentaires pour décider quels mots ou groupes de mots allaient être considérés comme des erreurs de français associées au clavardage lors de la correction des dictées. En effet, recueillir leurs commentaires s'est avéré utile, étant donné que cela ajoute un côté concret aux mots et aux groupes de mots traités comme des erreurs.

Les commentaires des adolescents concernant la langue requise en contexte de clavardage vont dans le sens des écrits recensés précédemment sur les caractéristiques de la langue codée associées au clavardage et aux textos. Notamment, les sujets disent faire appel à des réductions graphiques, où ils cherchent à couper des lettres dans un mot, et à des réductions avec variantes phonétiques, par le biais desquelles ils vont changer l'écriture des mots, tel que rapporté dans les typologies de Anis (2003) et de David et Goncalves (2007). Ces particularités ont donc été prises en compte lors de la correction de la dictée.

Aussi, certains élèves prennent à cœur le français et font attention à leur orthographe et à leur vocabulaire lorsqu'ils clavardent. Notamment, ces élèves précisent qu'ils font le moins de fautes possible; ils n'écrivent pas sous forme de langue codée et écrivent d'une 
façon correcte, non pas comme de nombreux adolescents. Ils font donc attention à leur écriture, mentionnent-ils. Il a même été souligné par un jeune qu'il cherche à utiliser des mots entiers, justement pour pratiquer son français écrit. Plusieurs font en sorte que le message demeure compréhensible, étant donné qu'ils ne comprennent pas toujours ce que les autres écrivent et qu'ils trouvent cela dérangeant, disent-ils. Par contre, la majorité des élèves n'utilisent pas un français convenable lorsqu'ils clavardent. Des élèves ont répondu à la question en plaçant une discussion en exemple; les mots employés dans leurs exemples se référent pour la plupart aux mots ciblés sur les sites de clavardage et aux caractéristiques de la langue codée recensées dans la problématique.

D'autres reconnaissent faire beaucoup d'erreurs de français par l'intermédiaire de ce média. Par exemple, des élèves ont répondu qu'ils écrivaient tout simplement avec beaucoup de fautes, en utilisant plusieurs abréviations et en écrivant tel que les mots se prononcent. Nous pouvons ici lier cette affirmation avec l'oral, puisque le mot s'écrit en contexte de clavardage comme il est prononcé oralement. Quelques adolescents avouent faire des fautes, mais demeurer attentifs à leur écriture, tandis que d'autres disent le contraire, c'est-à-dire qu'ils ne font pas du tout attention à leur écriture lorsqu'ils clavardent. Il a été mentionné par plusieurs jeunes qu'ils écrivent de la façon la plus courte possible, ne conjuguent pas leurs verbes, ne prennent pas le temps de taper les accents, n'écrivent pas les mots longs au complet et utilisent des expressions et des mots qui ne sont pas employés couramment dans la vie de tous les jours. Les jeunes expliquent qu'ils écrivent simplement à leur façon, tout en gardant à l'esprit que les mots doivent pouvoir être tapés le plus rapidement possible. D'autres ne font pas beaucoup de fautes, mais ne prennent pas le temps de se corriger. En fait, ils ne se révisent pas, ce qui va dans le sens des explications du cadre théorique concernant l'omission des stratégies d'écriture en situation de clavardage. Des élèves signalent aussi l'usage de mots anglais dans leurs écrits et l'utilisation d'un langage populaire. Les temps de verbe sont changés, les accords ne sont pas faits et les mots sont raccourcis et coupés. Par ailleurs, les lettres majuscules et minuscules sont mélangées; des élèves ont même rapporté que tous les utilisateurs font des erreurs, ce qui démontre cette culture de la langue codée reliée au clavardage, qui est 
semble-t-il bien ancrée dans l'esprit des adolescents d'aujourd'hui. Par ailleurs, un élève a spécifié qu'il enlève les $e$ lorsqu'il parle au je (jsuis) et qu'il fait appel à beaucoup de lettres doubles.

Ces affirmations, qui sont un résumé des commentaires des 158 adolescents, ont ainsi été prises en considération lors de la correction des dictées. Puisque les élèves affirment écrire de cette façon et que cela concorde avec les écrits recensés sur les caractéristiques de la langue codée en contexte de clavardage, nous considérons que nous pouvons nous fier à leurs commentaires pour discerner si les erreurs sont attribuables au clavardage ou non. Ainsi, l'annexe II consiste en un registre des erreurs, soit tous les mots et tous les groupes de mots où des erreurs ont été commises par les élèves lors de la dictée, erreurs qui ont été comptées comme étant des erreurs de français associées au clavardage dans la dictée. Dans ce registre sont indiqués les mots et les groupes de mots dans leur orthographe correcte, ainsi que les différentes erreurs constatées concernant chaque mot ou groupe de mots.

Il est enfin important de préciser que l'attention a été dirigée uniquement sur l'aspect orthographique de la langue écrite; les erreurs syntaxiques n'ont donc pas été prises en compte dans le cadre de cette étude.

\subsubsection{Questionnaire sur les habitudes informatiques}

Le questionnaire sur les habitudes informatiques est présenté en annexe III et compte plusieurs questions concernant le temps que consacrent les élèves au clavardage et l'utilisation qu'ils font de l'informatique. La variable temps hebdomadaire consacré au clavardage a été mesurée par le biais de ce questionnaire lorsque les jeunes ont eu à répondre à la question six. Nous n'avons pas suggéré aux élèves de façon de nommer le temps pour ce qui est de cette question où ils devaient indiquer le temps passé à clavarder chaque semaine; cela a donc été laissé à leur discrétion. Ainsi, ils étaient libres d'inscrire le temps en minutes ou en heures. Nous avons préféré cette formule pour ne pas influencer leurs réponses. Le temps a été de toute façon converti en minutes pour le traitement 
statistique des données et ensuite reconverti en heures pour les explications des résultats. Il s'agit d'une variable continue, puisque le temps hebdomadaire consacré au clavardage peut

prendre un grand nombre de valeurs. Également, les données concernant la variable âge de début du clavardage ont elle aussi été recueillies par l'intermédiaire de ce questionnaire. Cependant, cette question a été ajoutée par les élèves à la main, à la dernière minute, ce qui fait en sorte qu'une quantité inférieure de sujets (133) ont pris le temps d'y répondre. Pour terminer, les données relatives à la variable le fait que l'élève clavarde ou non ont aussi été considérées. Pour ce faire, la variable continue temps hebdomadaire consacré au clavardage a été convertie en variable dichotomique, selon qu'ils clavardent ou qu'ils ne clavardent pas.

\subsection{Methode D'Analyses Statistiques}

\subsubsection{Méthodologie et recherche quantitative}

Selon Crotty (1998), la méthodologie est définie comme étant « la stratégie, le plan d'action, le processus sous-jacent au choix et à l'application de techniques de travail spécifiques nommées méthodes. Elle fait le lien entre le choix des méthodes et les résultats attendus » (Crotty, 1998: 3). Ces éléments indiquent l'importance de choisir une méthodologie adaptée à l'objet de la recherche.

Dans le cadre de ce projet, les méthodes quantitatives ont été privilégiées. Celles-ci visent à réaliser une recherche par le biais d'hypothèses prédéterminées et de vérifier si elles seront validées ou non (Karsenti et Savoie-Sajc, 2004). En réalité,

[...] le but ultime de la recherche quantitative est de faire, à l'aide de données quantifiées, une démonstration éloquente d'une relation entre plusieurs variables. Pour faire valoir sa démonstration, le chercheur a la possibilité de présenter des tableaux qui résument les informations recueillies, de les illustrer à l'aide de figures ou de graphiques pour mettre en évidence les différences entre les résultats, puis à la suite de l'analyse statistique des données, de faire ressortir les relations 
entre les variables par une synthèse des résultats souvent présentée sous forme de tableau ou de schéma. (Karsenti et Savoie-Zajc, 2004 : 162)

\subsubsection{Position épistémologique}

La position épistémologique influence l'orientation de toute recherche. Dans le cadre de la rédaction de ce mémoire, le courant épistémologique préconisé est le positivisme. Il s'agit d'un courant qui favorise l'utilisation de données quantifiées, divisées en variables, lors de la cueillette et de l'analyse de données. Ainsi, la méthodologie de la recherche doit être de nature quantitative, ce qui est le cas dans l'actuelle recherche. Les données doivent également être observables et mesurables. En fait, selon le paradigme positiviste, les éléments de la recherche doivent être logiques et appuyés par une certitude scientifique. Le but premier du positivisme est de «prédire l'occurrence d'un phénomène par l'étude des causes qui entrainent des effets dans certaines conditions » (Karsenti et Savoie-Zajc, 2004 : 115). Pour les besoins de ce mémoire, l'influence du clavardage sur la maîtrise du français écrit est vérifiée, ce qui va dans le sens du courant positiviste, étant donné que nous présumons qu'une variable a une influence sur une autre, et que celles-ci seront appréhendées de façon quantitative. Concernant les caractéristiques du chercheur réalisant une telle recherche scientifique, celui-ci se démarque par son objectivité et par le fait qu'il ne laisse pas ses valeurs entrer en ligne de compte lors de la réalisation d'une étude rigoureuse (Karsenti et Savoie-Zajc, 2004).

\subsubsection{Statistiques descriptives}

Dans la section résultats, les statistiques descriptives de chaque variable seront présentées.

Dans l'ensemble, la variable âge de début du clavardage nous a permis de déterminer à quel âge les jeunes d'aujourd'hui, autant selon l'échantillon en entier que selon chaque sexe, débutent en moyenne à clavarder. Cette variable est de nature continue. Les 
statistiques descriptives ont contribué à préciser le nombre de sujets, la moyenne, l'écarttype, la médiane, le mode, le minimum et le maximum la concernant.

Ensuite, nous avons pu analyser la variable temps hebdomadaire consacré au clavardage, en établissant la moyenne des heures par semaine consacrées au clavardage pour l'échantillon au complet et d'après le sexe des sujets ensuite, afin de vérifier si les filles passent plus de temps à clavarder à chaque semaine que les garçons. Le temps hebdomadaire consacré au clavardage est également une variable continue. Les mesures de tendance centrale et de dispersion citées pour l'âge de début du clavardage seront également spécifiées pour le temps hebdomadaire consacré au clavardage.

Par la suite, la même démarche a été effectuée avec la variable le fait que l'élève clavarde ou non, dans l'objectif de vérifier combien d'élèves clavardent comparativement à ceux qui ne clavardent pas. Il a aussi été vérifié si plus de filles que de garçons s'adonnent au clavardage. Concernant cette dernière variable, celle-ci est une variable catégorielle dichotomique, étant donné qu'elle possède deux valeurs seulement (clavarde et ne clavarde pas). Pour cette variable, les fréquences en chiffres et en pourcentage seront présentées.

Concernant la variable dépendante, soit les erreurs de français associées au clavardage, les mesures de tendance centrale et de dispersion prises en compte sont le nombre de sujets, la moyenne, l'écart-type, la médiane, le mode, le minimum et le maximum, compte tenu du fait qu'il s'agit d'une variable continue. De plus, les statistiques

descriptives des filles et des garçons seront comparées en ce qui a trait à cette dernière variable.

\subsubsection{Statistiques corrélationnelles}

Dans le cadre de la présente recherche, différents tests statistiques ont été effectués dans le but de vérifier l'hypothèse principale, qui suppose que le clavardage a une influence défavorable sur la maîtrise du français écrit des élèves du premier cycle du secondaire. 
Pour débuter, dans l'intention de vérifier la première hypothèse secondaire stipulant que plus les élèves débutent à clavarder à un jeune âge, plus ils sont susceptibles de faire des erreurs de français associées au clavardage, une analyse de régression linéaire a été réalisée, ce qui a permis de vérifier s'il y a un lien significatif entre les deux variables. $\mathrm{La}$ régression a été privilégiée, étant donné qu'elle vise à vérifier le lien entre deux variables continues lorsqu'il est présumé qu'une variable a un effet sur une autre (Howell, 2008). Les variables prises en considération sont ici l'âge de début du clavardage, qui est la variable indépendante, ainsi que les erreurs de français associées au clavardage, qui est la variable dépendante.

Ensuite, la seconde hypothèse à analyser était la suivante : plus les élèves clavardent durant la semaine, plus ils sont susceptibles de faire des erreurs de français associées au clavardage. Pour ce faire, une analyse de régression a permis de déterminer s'il y a un lien significatif entre les variables continues temps hebdomadaire consacré au clavardage et erreurs de français associées au clavardage.

Pour terminer, il a été vérifié, par l'intermédiaire d'un test $t$, qui vise à vérifier le lien entre une variable dichotomique et une variable continue (Howell, 2008), si le fait que l'élève clavarde ou non a une influence sur les erreurs de français associées au clavardage.

Ces trois hypothèses contribueront à confirmer ou à infirmer l'hypothèse principale.

\subsection{Conclusion}

Ce chapitre visait à expliquer chaque élément relatif à la méthodologie associée à cette recherche. Le prochain chapitre présente de façon détaillée les résultats obtenus à l'expérimentation. 


\section{CHAPITRE 5}

\section{RÉSULTATS}

\subsection{INTRODUCTION}

Dans ce chapitre seront présentés les différents résultats associés à ce mémoire de maîtrise, dont l'objectif est de déterminer si le clavardage a une influence sur la maîtrise du français écrit des élèves du premier cycle du secondaire.

En tout premier seront abordées la question de recherche et les hypothèses s'y rattachant. Par la suite, les statistiques descriptives de chaque variable seront présentées. Les analyses statistiques corrélationnelles suivront. La discussion, qui consiste en l'interprétation des résultats, permettra ensuite de clore ce chapitre.

\subsection{QUESTION DE RECHERCHE ET HYPOTHESES}

La question de recherche se pose comme suit: quelle est l'influence du clavardage sur la maitrise du français écrit des élèves du premier cycle du secondaire? Dans le but de répondre à cette question, l'hypothèse principale suppose que le clavardage a une influence défavorable sur la maîtrise du français écrit des élèves du premier cycle du secondaire. Afin de la vérifier, des hypothèses secondaires issues de cette dernière ont été formulées. Les voici :

- Plus les élèves débutent le clavardage à un jeune âge, plus ils sont susceptibles de faire des erreurs de français associées au clavardage; 
- Plus les élèves clavardent durant la semaine, plus ils sont susceptibles de faire des erreurs de français associées au clavardage;

- Les élèves qui clavardent font davantage d'erreurs de français associées au clavardage comparativement à ceux qui ne clavardent pas.

\subsection{Statistiques DESCRIPTIVES}

\subsection{1 Âge de début du clavardage}

Lors du questionnaire sur les habitudes informatiques, les élèves ont eu à répondre à quelques questions concernant l'usage qu'ils font de l'ordinateur. Notamment, ils devaient répondre à la question suivante : à quel âge as-tu commencé à clavarder? Il est nécessaire de préciser que 135 élèves seulement ont répondu à cette question, étant donné que les sujets ont dû l'ajouter à la main sur le questionnaire.

L'analyse des réponses à cette question montre que les jeunes $(\mathrm{N}=135)$ commencent en moyenne à clavarder à 9,36 ans. La médiane et le mode sont de 9, alors que l'écart-type est de 1,6I. L'âge minimum de début du clavardage est 4 ans et l'âge maximum est 13 ans. La variable âge de début du clavardage est une variable continue, étant donné qu'elle peut emprunter un assez grand nombre de valeurs entre 4 et 13. La figure 1 représente la distribution de l'âge de début du clavardage des élèves de l'échantillon. 


\section{Histogramme}

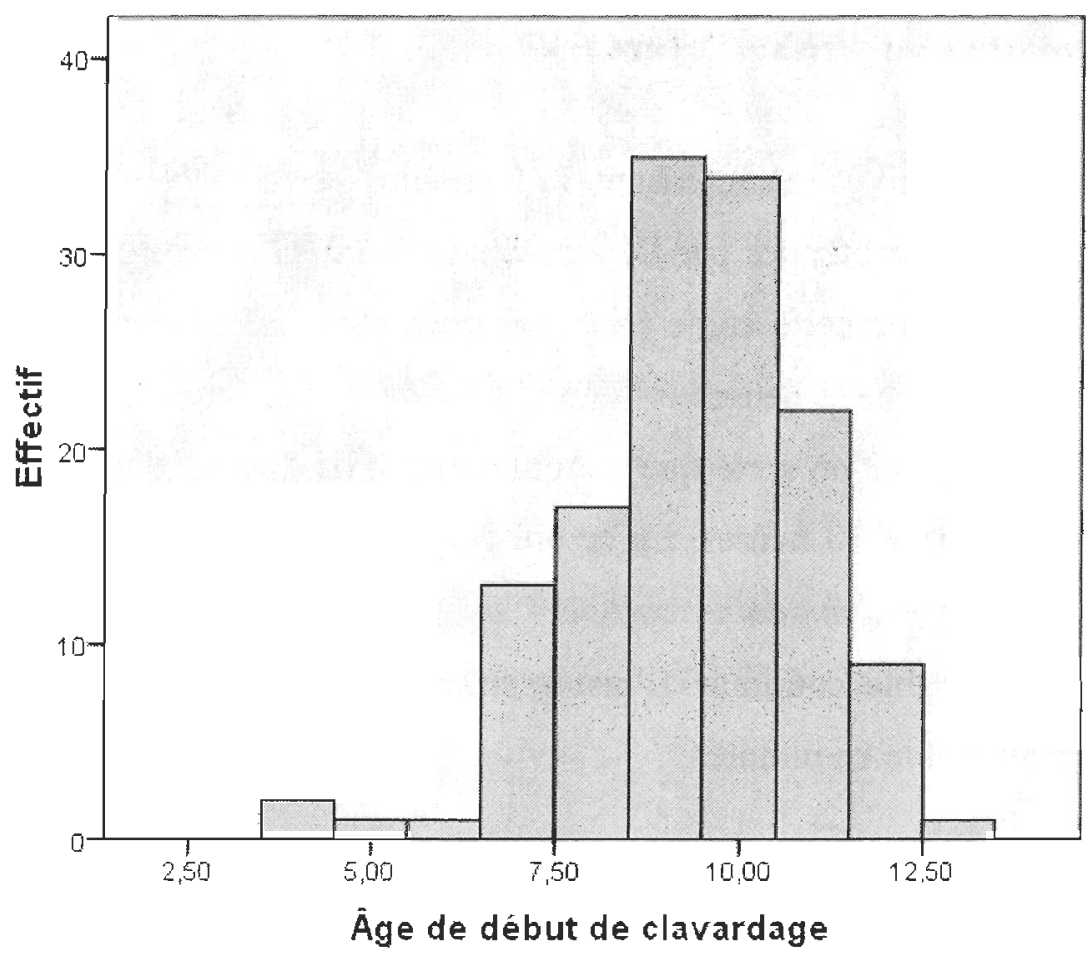

$\begin{aligned} & \text { Moyenne }=9: 36 \\ & \text { Ecart type }=1: 605\end{aligned}$

$N=135$

Figure 1: Âge de début du clavardage

Il est aussi intéressant de comparer l'âge de début du clavardage selon le sexe des sujets. Sur les 135 sujets ayant répondu à cette question, les filles $(N=61)$ ont débuté en moyenne à clavarder à 9,64 ans, avec un écart-type de 1,14, tandis que les garçons $(\mathrm{N}=74)$ ont commencé à le faire à l'âge de 9,14 ans, avec un écart-type de 1,88. À première vue, la différence semble minime, puisqu'elle est de 0,5 seulement. Un test $t$ a tout de même permis de vérifier s'il y a une différence significative entre les filles et les garçons à cet égard. Il s'agit d'un test statistique qui permet de comparer deux moyennes entre elles, tel que requis pour la présente analyse. La probabilité au test de Levene étant de 0,001 , le test $t$ corrigé a été pris en considération. Les résultats de ce test montrent que : t $(122,86)=1,917$ $p=0,058$. Nous pouvons constater que la probabilité est plus élevée que 0,05 . Nous 
pouvons alors affirmer qu'il n'y a pas de différence significative entre les garçons et les filles en ce qui a trait à l'âge de début du clavardage.

\subsubsection{Temps hebdomadaire consacré au clavardage}

Voici les statistiques descriptives caractérisant la variable temps hebdomadaire consacré au clavardage, qui ont été obtenues par l'intermédiaire du questionnaire sur les habitudes informatiques. Les sujets de cette étude $(\mathrm{N}=158)$ clavardent en moyenne 4,30 heures par semaine. La médiane est de 2 heures et le mode est de 0 . Plusieurs élèves ne clavardent donc pas du tout, ce qui fait en sorte que le minimum est de 0 . En revanche, le temps de clavardage maximum est de 50 heures. En ce qui concerne l'écart-type, celui-ci est de 6,32. La variable temps hebdomadaire consacré au clavardage est elle aussi caractérisée comme étant une variable continue. L'histogramme représenté à la figure 2 illustre la distribution de cette variable en minutes. 


\section{Histogramme}

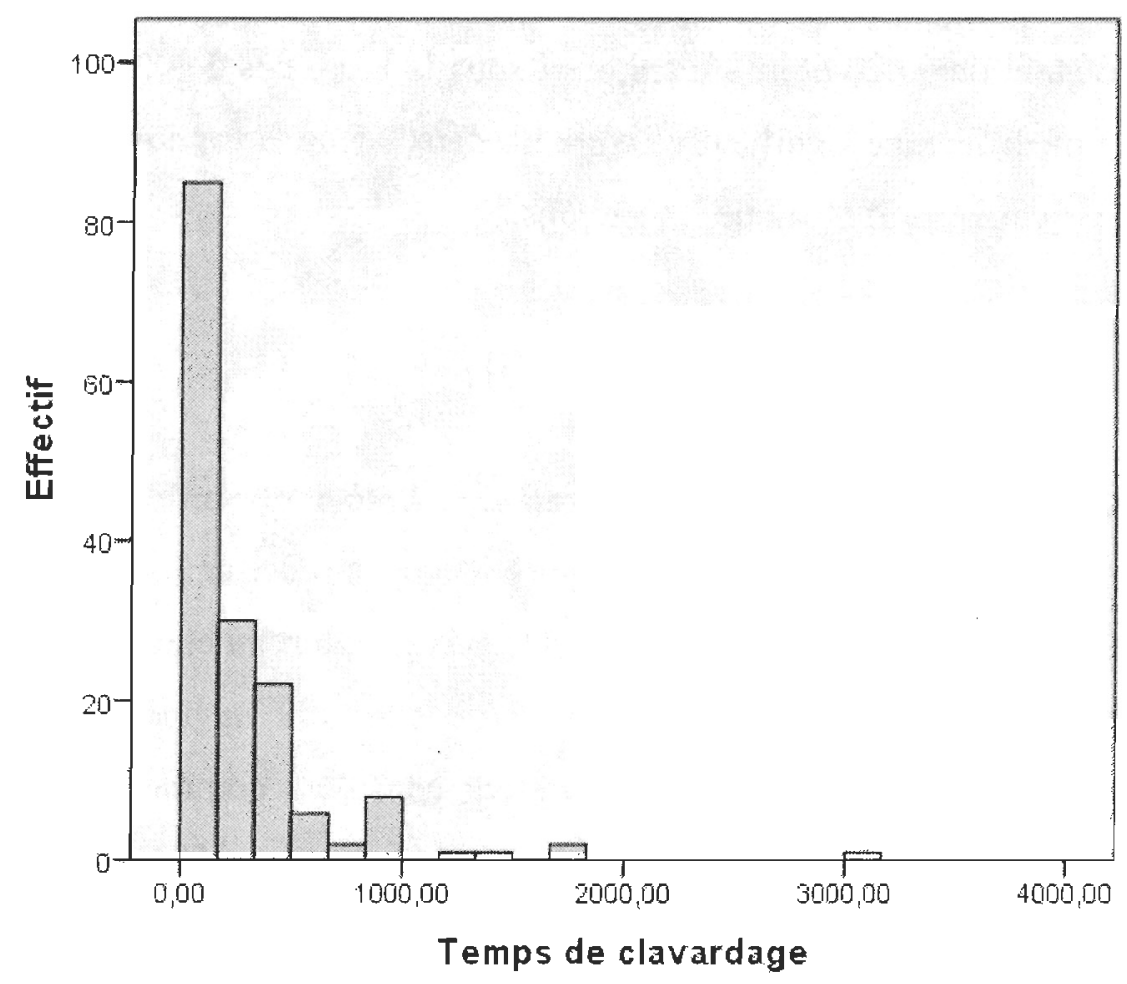

Moyenne $=258,89$
Ecart type $=37,184$ N $=158$

Figure 2 : Temps hebdomadaire consacré au clavardage

Par ailleurs, il est à noter que les garçons $(N=90)$ clavardent 3,28 heures par semaine, avec un écart-type de 4,80 , alors que les filles $(\mathrm{N}=68)$ se consacrent au clavardage 5,70 heures par semaine. En ce qui les concerne, l'écart-type est de 7,72. Au premier abord, on constate que les filles clavardent plus que les garçons, puisqu'il y a une différence qui semble évidente entre les deux sexes. En effet, cette différence est de 2,42 heures par semaine.

Dans le but de comparer la variable sexe des sujets selon le temps hebdomadaire consacré au clavardage, un test $\mathrm{t}$ a été réalisé. Tel que précisé précédemment, ce test vise à comparer entre elles deux moyennes, ce qui permettra de déterminer si elles s'écartent significativement l'une de l'autre. 
Pour ce qui est des résultats au test de Levene, la probabilité (p) est de 0,005. Ainsi, puisque la probabilité est inférieure à $5 \%$, le test t corrigé a été considéré. Les résultats à ce test sont : $\mathrm{t}(105,06)=-2,256, \mathrm{p}=0,026$.

La probabilité étant de 0,026 et se situant ainsi sous la barre des $5 \%$, nous pouvons confirmer qu'il y a une différence significative entre les deux sexes. Il est donc vrai que les filles clavardent significativement plus que les garçons.

\subsubsection{Le fait que l'élève clavarde ou non}

Par le biais de cette recherche, il a été déterminé si l'élève clavarde ou non. Étant donné qu'il a répondu à un questionnaire lors duquel il devait préciser le temps passé à clavarder chaque semaine, il a été possible d'établir si l'élève recourt au clavardage ou non. Pour ce faire, la variable continue temps hebdomadaire consacré au clavardage a été classifiée en variable catégorielle dichotomique, 0 étant considéré comme «ne clavarde pas » et les autres temps étant considérés comme «clavarde». Sur les 158 participants qui ont répondu au questionnaire, 135 d'entre eux s'adonnent au clavardage, ce qui représente $85,44 \%$ de l'échantillon, contre 23 élèves (14,56\%) qui ne clavardent pas du tout.

Par ailleurs, les résultats indiquent que 74 garçons clavardent, alors que 16 ne le font pas. Pour ce qui est des filles, 61 clavardent, tandis que sept d'entre elles ne recourent pas du tout à ce mode de communication. Dans le but de vérifier s'il existe une différence significative entre les garçons et les filles en ce qui a trait à la variable le fait que l'élève clavarde ou non, un test du khi-carré a été réalisé. Les résultats au test $\left(\mathrm{x}^{2}=1,744(1), \mathrm{p}=\right.$ $0,187)$ ont révélé qu'il y a pas de différence significative entre les garçons et les filles concernant le fait qu'ils clavardent ou non. Ils le feraient donc dans des proportions relativement semblables. En effet, on constate à la lumière de ces chiffres que $82,22 \%$ des garçons clavardent, alors que $89,70 \%$ des filles le font. 


\subsubsection{Erreurs de français associées au clavardage}

Abordons les statistiques descriptives en relation avec la variable erreurs de français associées au clavardage, qui constitue la variable dépendante. Les 158 sujets ont commis en moyenne 4,91 erreurs lors de la dictée, avec un écart-type de 4,12. L'analyse des données révèle que la médiane est 4 , alors que le mode se situe à 3 . Le nombre d'erreurs minimum est 0 , tandis que les erreurs de l'élève en ayant accompli le plus se comptent à 24 . Cette variable est de nature continue, étant donné qu'elle peut prendre un grand nombre de valeurs entre 0 et 24. La figure 3 illustre sur un histogramme la distribution des erreurs de français associées au clavardage dans cet échantillon. En observant cette figure, nous pouvons constater qu'un grand nombre d'élèves a effectué entre 3 et 5 erreurs.

\section{Histogramme}

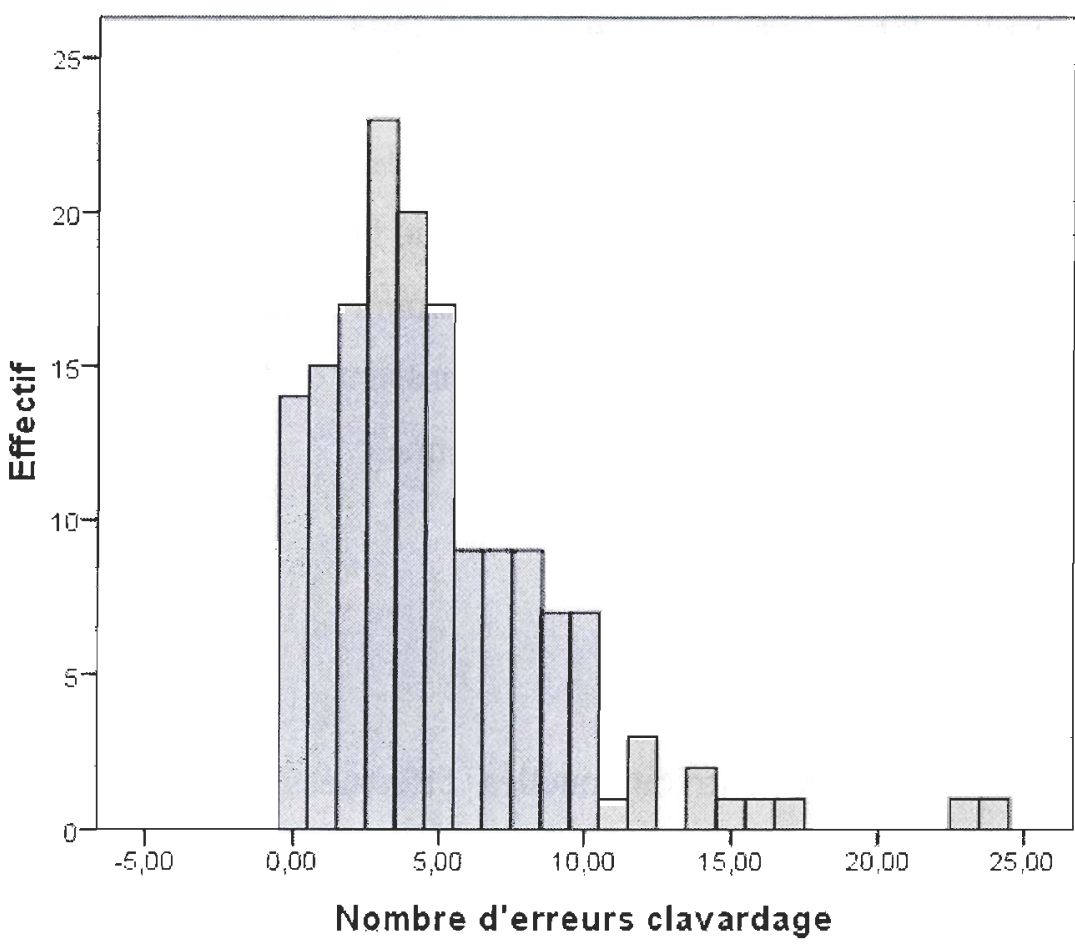

Moyenne $=491$ Ecalt type $=4,117$ $N=155$

Figure 3 : Erreurs de français associées au clavardage 
Il est intéressant de comparer les erreurs de français associées au clavardage des garçons et des filles, et ce, dans le but d'examiner s'il y a un écart significatif à cet égard. Notamment, les garçons ( $\mathrm{N}=90)$ commettent en moyenne 6,11 erreurs, avec un écart-type de 4,49. Les filles $(N=68)$ font quant à elles 3,31 erreurs seulement. L'écart-type les concernant est de 2,90. Suite à la lecture de ces chiffres, nous pouvons aisément remarquer que les garçons font davantage d'erreurs de français associées au clavardage que les filles, étant donné qu'il y a une différence de 2,80 points entre les deux sexes. En revanche, il est nécessaire d'effectuer un test $t$ pour vérifier la véracité de cette affirmation.

Au départ, la probabilité au test de Levene était de 0,006 , ce qui fait en sorte que le test $t$ corrigé a été priorisé, compte tenu que la probabilité se situe sous la barre des $5 \%$. Les résultats montrent que $\mathrm{t}(152,67)=4,75, \mathrm{p}=0,000$. Puisque la probabilité est de $0 \%$, il est donc possible d'affirmer que les garçons font significativement plus d'erreurs de français associées au clavardage que les filles dans cet échantillon.

Enfin, à titre indicatif, un test de corrélation, qui a pour objectif de vérifier le lien entre deux variables continues, a permis de vérifier si les élèves qui ont commencé à clavarder à un jeune âge passent plus de temps à clavarder que ceux qui ont commencé à le faire plus tard. Celui-ci $(\mathrm{r}=-0,249, \mathrm{p}=0,004)$ démontre que la corrélation est significative dans le sens inverse, ce qui fait en sorte que c'est effectivement le cas, c'est-à-dire que plus les élèves ont débuté à clavarder à un jeune âge, plus ils passent de temps à le faire chaque semaine. La force d'intensité de cette corrélation est moyenne.

\subsection{Statistiques CORRELATIONNELLES}

Voici maintenant les analyses corrélationnelles effectuées, qui visent à vérifier l'hypothèse principale de différentes façons selon les données traitées. Cette hypothèse stipule que le clavardage a une influence défavorable sur la maîtrise du français écrit des élèves du premier cycle du secondaire 


\subsubsection{Lien entre l'âge de début du clavardage et les erreurs de français associées au clavardage}

Il est de circonstance d'examiner l'hypothèse qui suppose que plus les élèves débutent le clavardage à un jeune âge, plus ils sont susceptibles de faire des erreurs de français associées au clavardage.

Dans le but d'établir si cette hypothèse est confirmée ou non, un test de régression linéaire a été réalisé. Ce test a pour objectif de vérifier s'il y a un lien significatif entre l'âge de début du clavardage et les erreurs de français y étant associées, considérant que cette première variable a une influence sur la seconde. La variable indépendante est donc l'âge de début du clavardage et la variable erreurs de français associées au clavardage est la variable dépendante.

En somme, les résultats à l'analyse de régression $(r=0,01, p=0,904)$ démontrent qu'il n'y a pas de lien significatif entre les deux variables, ce qui fait en sorte que l'hypothèse est infirmée, c'est-à-dire que les élèves faisant usage du clavardage depuis plus longtemps ne font pas plus d'erreurs que ceux qui ont recours à ce média depuis peu. Cet état de la situation a été démontré par la valeur de la probabilité, qui est supérieure à 0,05 , ce qui permet de dire avec certitude qu'un lien n'est pas significativement observable entre ces deux variables. Le diagramme de dispersion de la figure 4 représente la situation. En s'y attardant, nous pouvons facilement constater l'inexactitude de l'hypothèse formulée, puisque la droite de régression est à l'horizontale et demeure pratiquement au même niveau, peu importe l'âge de début du clavardage en fonction du nombre d'erreurs. 


\section{Nombre d'erreurs clavardage}

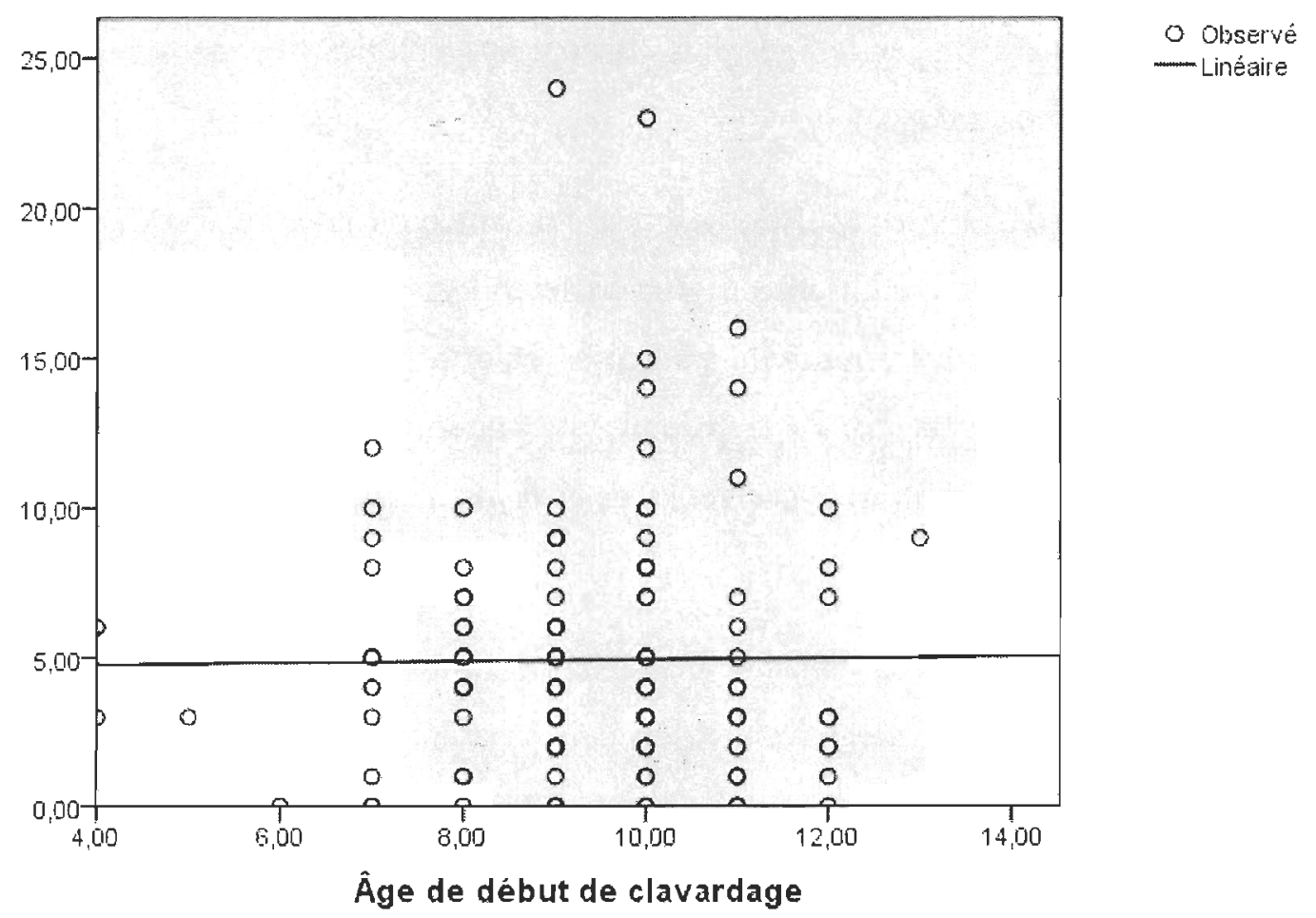

Figure 4 : Erreurs de français associées au clavardage selon l'âge de début du clavardage

\subsubsection{Lien entre le temps hebdomadaire consacré au clavardage et les erreurs de français associées au clavardage}

Dans le but de vérifier l'hypothèse qui propose que plus les élèves clavardent durant la semaine, plus ils sont susceptibles de faire des erreurs de français associées au clavardage, une analyse de régression linéaire a été effectuée. Tel que mentionné précédemment, la régression vise à vérifier le lien entre deux variables continues lorsqu'il est présumé qu'une variable a une incidence sur l'autre. Les deux variables continues analysées sont le temps hebdomadaire consacré au clavardage et les erreurs de français associées au clavardage. Les résultats à l'analyse sont les suivants : $r=0,019, p=0,816$. 
Pour qu'une hypothèse soit confirmée, la probabilité doit être égale ou inférieure à $5 \%$, ce qui n'est pas le cas actuellement, étant donné que celle-ci se chiffre à $81,6 \%$. Cette analyse statistique nous montre donc que le temps hebdomadaire consacré au clavardage n'influence pas négativement la qualité du français écrit du groupe d'âge ciblé.

Le diagramme de dispersion présenté à la figure 5 illustre cette régression. Le temps hebdomadaire est indiqué en minutes. Lorsque nous étudions la droite de régression, nous constatons qu'elle n'est pas très prononcée; elle est même presque horizontale, ce qui démontre encore une fois qu'il n'y a pas de lien significatif entre les deux variables analysées.

\section{Nombre d'erreurs clavardage}

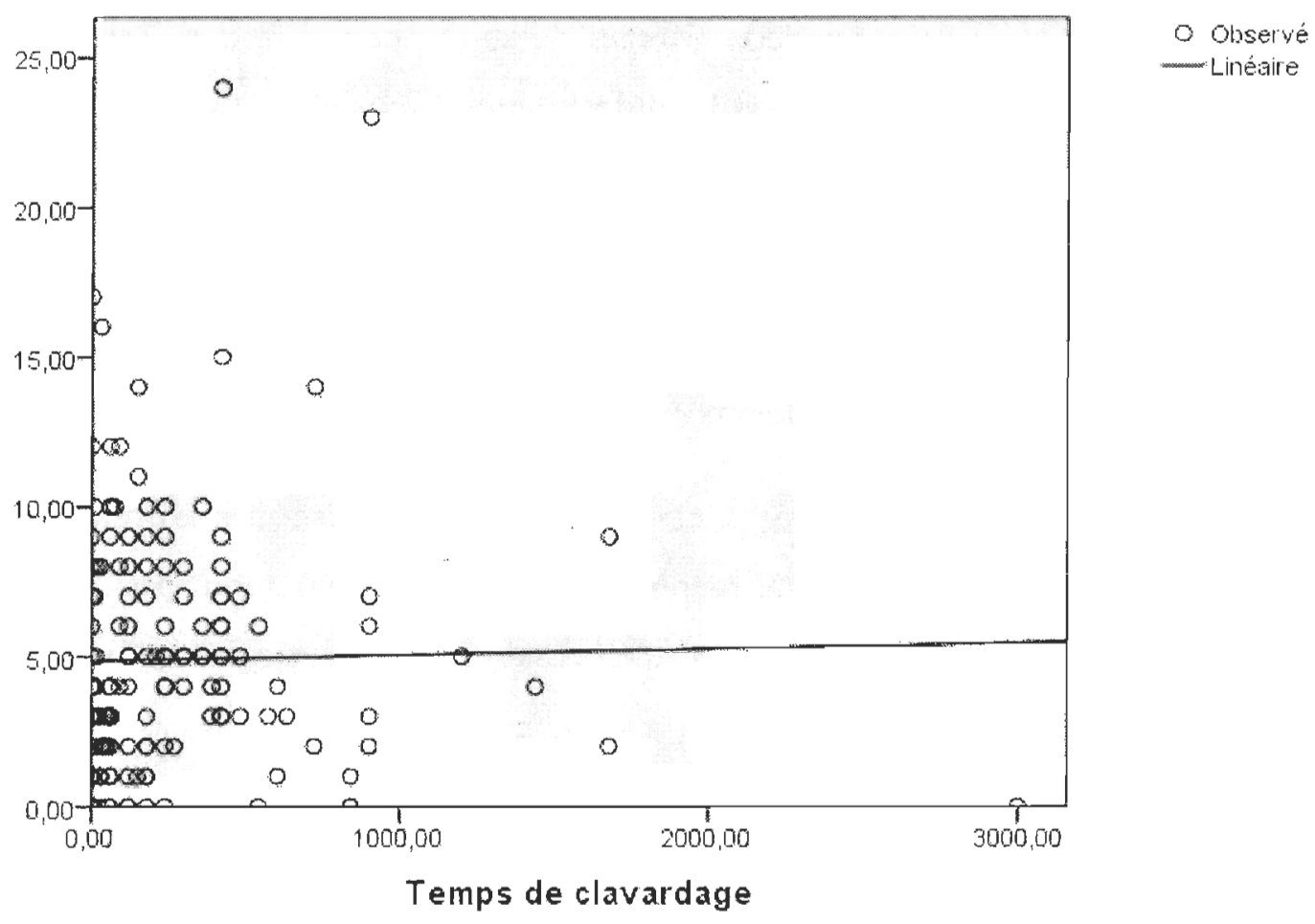

Figure 5 : Erreurs de français associées au clavardage selon le temps hebdomadaire consacré au clavardage 


\subsubsection{Lien entre le fait que l'élève clavarde ou non et les erreurs de français associées au clavardage}

Il est nécessaire de comparer les erreurs de français associées au clavardage selon le fait que l'élève clavarde ou non, dans le but d'établir si les élèves qui clavardent font davantage d'erreurs de français associées au clavardage comparativement à ceux qui ne clavardent pas.

Pour ce faire, un test $\mathrm{t}$ a été appliqué, dans le but de comparer les deux moyennes entre elles afin de vérifier si l'hypothèse sera confirmée. En outre, les analyses montrent que parmi les 135 sujets utilisant le clavardage comme outil de communication, ceux-ci ont effectué en moyenne 5 erreurs lors de la dictée, avec un écart-type de 4,13. Pour ce qui est des jeunes qui ne clavardent pas du tout, qui sont au nombre de 23 , ils y ont fait quant à eux 4,35 erreurs. L'écart-type est alors de 4,10. À première vue, nous pouvons constater que la différence semble minime entre les deux groupes de sujets, ce qui irait en contresens de l'hypothèse rédigée. Les résultats au test $\mathrm{t}$ valident cette observation : $\mathrm{t}(156)=0,70, \mathrm{p}=$ 0,484 .

Puisque la probabilité est supérieure à $5 \%$, l'hypothèse n'est pas confirmée. Il n’y a donc pas de différence significative à l'égard des erreurs de français associées au clavardage entre les élèves qui clavardent et ceux qui ne s'y prêtent pas du tout.

En somme, les résultats obtenus à ces trois analyses statistiques corrélationnelles infirment l'hypothèse principale, ce qui montre que le clavardage n'aurait pas d'influence défavorable sur la maîtrise du français écrit des élèves du premier cycle du secondaire.

\subsection{DisCUSSION DES RESULTATS}

La discussion, soit l'interprétation des résultats, consiste en une réflexion sur les différents résultats obtenus. Pour ce faire, les hypothèses formulées de même que les résultats s'y rattachant seront d'abord rappelés. Dans l'objectif de vérifier l'hypothèse 
principale selon plusieurs aspects, il a été jugé de circonstance de réaliser plusieurs analyses. Ces analyses ont permis de découvrir de nouvelles statistiques associées à ce mode de communication priorisé par les jeunes d'aujourd'hui. De plus, il sera précisé si les résultats confirment ou non ceux des études consultées dans le cadre de la recension des écrits de ce mémoire de maitrise. Ces résultats seront également liés au cadre théorique, donc à la psychologie cognitive. Il importe aussi de préciser que des analyses concernant le clavardage, mais qui ne visent pas à vérifier l'hypothèse principale, ont également été effectuées; elles permettent de bien cerner les particularités du clavardage, notamment par rapport au sexe des sujets. Enfin, les limites de l'étude seront développées.

Revoici l'hypothèse principale: le clavardage a une influence défavorable sur la maîtrise du français écrit des élèves du premier cycle du secondaire.

Avant l'analyse des données, nous étions ambivalentes quant aux résultats possibles. Suite aux lectures effectuées sur le sujet, nous avons eu l'occasion de constater que les opinions concernant l'objet de la présente recherche sont mitigées. En effet, certains auteurs et autres professionnels de l'éducation sont d'avis que le clavardage n'a pas d'impact négatif sur la maîtrise de la langue, tandis que d'autres voient son essor avec une certaine inquiétude. Particulièrement, Ouellon (2009) précise que le français ne doit pas s'inquiéter de la langue codée employée en contexte de clavardage et que cette langue récente illustre plutôt que les jeunes d'aujourd'hui sont capables de s'adapter à la manipulation de deux formes de langue bien distinctes, qui varient selon les circonstances. Il est d'accord pour dire que ce nouveau répertoire n'aide pas les jeunes à acquérir les bonnes bases de la langue française, mais qu'il n'y nuit pas pour autant. D'ailleurs, il mentionne que les jeunes sont capables de distinguer quand ils doivent recourir à chaque forme d'écriture. Du côté de la Belgique, Fairon (2007) est d'avis que les jeunes sont capables d'écrire selon plusieurs langues et de cerner quand chaque forme de français est requise. Toutefois, Chouinard (2002) a ressorti les propos de Simard, qui a déjà agi comme ministre de l'Éducation. Il a pour opinion qu'il faut s'attendre à retrouver certains mots du clavardage dans les écrits scolaires des adolescents et qu'il est important de sensibiliser la population face à cette 
nouvelle méthode de communication. De plus, les questionnements à cet égard des différents enseignants interrogés de façon informelle, qui manifestent une certaine inquiétude quant à l'utilisation du clavardage, sont venus rejoindre nos propres réflexions par rapport à ce nouveau moyen de communication. Tous ces éléments démontrent le bienfondé de la recherche entreprise, puisqu'il y a un questionnement évident quant à l'influence de ce phénomène sur la maîtrise de la langue écrite.

Tout d'abord, l'hypothèse suivante a été vérifiée: plus les élèves débutent le clavardage à un jeune âge, plus ils sont susceptibles de faire des erreurs de français associées au clavardage. À cette fin, un test de régression linéaire a été appliqué. Les résultats au test ont démontré que l'âge de début du clavardage n'influence pas le nombre d'erreurs de français associées au clavardage, étant donné que les élèves ayant débuté à clavarder à un jeune âge n'ont pas fait plus d'erreurs que d'autres pour qui il s'agit d'un passe-temps beaucoup plus récent.

En ce qui nous concerne, nous avons été en tout premier surprises quant aux résultats à cette dernière analyse. En effet, nous pensions que les élèves qui clavardent depuis un plus jeune âge feraient davantage d'erreurs que ceux ayant commencé à le faire plus tard. Assurément, les élèves ayant débuté à clavarder plus tôt ont, pour la majorité d'entre eux, plus d'heures de clavardage à leur actif que ceux ayant débuté à le faire ultérieurement. Ils auraient donc pu automatiser le code de la langue associée au clavardage bien avant leurs pairs. Toutefois, le fait que l'âge de début du clavardage n'ait pas d'influence sur le nombre d'erreurs semble indiquer tout simplement que le clavardage n'a pas d'influence négative sur la maîtrise du français écrit des élèves du premier cycle du secondaire, tel que le montrent les résultats des autres analyses.

Afin de vérifier si plus les élèves clavardent durant la semaine, plus ils sont susceptibles de faire des erreurs de français associées au clavardage, une analyse de régression linéaire a été réalisée. Les résultats au test statistique révèlent que le temps hebdomadaire consacré au clavardage n'influence pas négativement la maîtrise du français écrit des élèves du premier cycle du secondaire, étant donné que les élèves qui passent 
beaucoup de temps à clavarder n'ont pas fait significativement plus d'erreurs que ceux qui clavardent peu ou pas du tout; ainsi, les jeunes qui clavardent par exemple 20 heures par semaine n'ont pas fait davantage de fautes que ceux qui clavardent seulement 5 heures, ce qui va dans le sens contraire de l'hypothèse de départ. Il est également important de mentionner que le nombre d'erreurs par copie varie énormément selon les cas. À dire vrai, certains élèves n'ont fait aucune faute liée au clavardage, tandis que d'autres en ont commis une vingtaine. En outre, le diagramme de dispersion présenté à la figure 5 montre que cela n'est pas expliqué par le temps hebdomadaire consacré au clavardage.

D'un autre côté, les résultats des autres études réalisées sur le sujet sont semblables à ceux de la présente recherche. Principalement, une étude anglophone (Varnhagen et al., 2009) visant à déterminer si le clavardage a une influence sur la langue a été réalisée récemment. Cette étude avait également pour objectif d'explorer la nature des mots produits par le clavardage ainsi que leurs fonctions, et d'examiner les relations entre l'habileté d'épeler et l'utilisation de cette nouvelle langue. L'étude démontre que les jeunes anglophones qui recourent au clavardage peuvent épeler de façon adéquate les mots qu'ils utilisent lors des séances de clavardage, ce qui suggère que celui-ci n'a pas d'effet défavorable sur la langue conventionnelle anglaise. 11 importe tout de même de spécifier qu'il s'agit d'une étude basée sur des tests d'épellation, et non pas sur des épreuves d'écriture. Nous pouvons malgré cela constater que les résultats de cette étude anglophone basée sur l'épellation sont semblables à ceux de notre étude dont les objectifs de base demeurent similaires.

De même, le fait que les élèves soient aptes à ne pas transférer le code du clavardage dans leurs écrits scolaires démontre qu'ils possèdent pour la majorité de bonnes aptitudes métacognitives. La plupart des élèves ont en effet fait la part des choses et ont écrit les mots de la façon dont ils leur ont été enseignés, et non pas comme ils les écrivent en clavardant. La métacognition semble donc avoir une influence sur la capacité des jeunes à ne pas transférer les écrits du clavardage dans les écrits conventionnels, tel que le supposent les explications du cadre théorique. Les jeunes ont ainsi différencié les contextes d'utilisation 
des deux langues. Par ailleurs, le système cognitif donne la possibilité à l'apprenant d'utiliser ses connaissances au bon moment, autant en contexte de clavardage qu'en contexte scolaire. Comme le précise Tardif (1992), ces stratégies permettent à l'élève de poser des actions concrètes et adaptées à la tâche. Par exemple, l'élève, lorsqu'il verbalise mentalement sa réflexion au cours d'une tâche, fait appel à ses stratégies cognitives. Ainsi, celles-ci peuvent l'aider à distinguer quand il doit recourir à chaque forme de français, ce qui fait en sorte que ces stratégies lui permettent d'écrire les mots de la dictée de manière adéquate.

Au fil de leur scolarité, les adolescents ont fini par acquérir un vocabulaire et les bases de l'écriture. Toutefois, lorsqu'ils clavardent, ils ne font pas toujours appel à ce vocabulaire, puisqu'ils désirent faire partie de la communauté virtuelle et ainsi utiliser la langue la caractérisant. Ils choisissent donc volontairement de ne pas user de la langue française conventionnelle. Étant donné qu'ils emploient les mots codés du clavardage peutêtre plus souvent que ceux du français conventionnel, nous nous sommes demandées s'ils ne finiront pas par automatiser ces mêmes mots et seraient ainsi portés à les écrire automatiquement dans les écrits scolaires. Les résultats démontrent finalement que ce n'est pas le cas, malgré le fait que plusieurs jeunes clavardent énormément durant une seule et même semaine. En revanche, il est pertinent de s'interroger concernant les années à venir. Les jeunes auront de plus en plus d'heures de clavardage à leur actif et clavarderont depuis plus longtemps, ce qui peut peut-être augmenter leurs chances de transfert dans des écrits qui doivent respecter les normes de la langue.

Le transfert des apprentissages est également un concept important du cadre théorique. Nous sommes conscientes que les jeunes ne prennent pas la peine de transférer les écrits du français conventionnel dans leurs écrits virtuels lorsqu'ils clavardent, mis à part pour quelques mots, bien entendu. Il est effectivement facile de constater que les adolescents n'emploient pas un français convenable lorsqu'ils communiquent virtuellement. Alors, si tous ces éléments sont considérés et que l'élève ne transfère pas le bon français en situation de clavardage, il n'est finalement pas étonnant que les jeunes ne 
transfèrent pas les écrits du clavardage dans leurs écrits scolaires. La métacognition doit ici être prise en considération puisqu'il est possible que l'absence volontaire de transfert soit due aux habiletés métacognitives des jeunes.

Dans le cadre de cette étude, il a été remarqué par l'intermédiaire d'un test t que le fait que l'élève clavarde ou non n'a pas d'incidence non plus sur le nombre d'erreurs de français associées au clavardage. Nous pouvons donc affirmer que les élèves qui clavardent ne font pas davantage d'erreurs comparativement à ceux qui ne se consacrent pas du tout à ce mode de communication. Nous pouvons lier ces résultats à une étude qui a été réalisée par Lafontaine et al. (2005). Notamment, l'objectif de cette recherche était de comparer entre elles les performances d'écriture en français des élèves qui utilisent le clavardage comme outil de communication de ceux qui n'y font pas du tout appel. Les résultats de cette recherche possèdent eux aussi plusieurs similitudes avec les nôtres. En fait, il a été démontré par les chercheurs, cela par l'intermédiaire d'une dictée, d'un questionnaire et d'expressions à corriger, que le clavardage n'a en définitive pas d'influence négative sur le français écrit des adolescents de cinquième secondaire. Les résultats au test $t$ ont en effet attesté qu'il n'y a pas de différence significative à cet égard entre les deux clientèles, soit les clavardeurs et les non-clavardeurs. Manifestement, si le temps hebdomadaire consacré au clavardage n'a pas d'incidence sur les erreurs de français associées au clavardage, il est logique de penser que le fait que l'élève clavarde ou non n'aura lui non plus d'incidence sur le nombre d'erreurs de français associées au clavardage. Pour revenir à l'âge de début du clavardage, si l'élève qui clavarde ne fait pas plus de fautes que celui qui ne clavarde pas, il est légitime de penser que l'âge de début du clavardage n'aura lui non plus d'influence sur le nombre d'erreurs.

Il est aussi intéressant d'étudier si le nombre d'erreurs de français associées au clavardage diffère de façon significative selon le sexe. Donc, dans le but de vérifier le lien entre les variables erreurs de français associées au clavardage et sexe des sujets, un test t a été réalisé, afin de confirmer si les garçons font davantage d'erreurs reliées au clavardage que les filles. En outre, les résultats au test t confirment cette affirmation et révèlent que les 
garçons ont effectivement commis plus d'erreurs que les filles. D'ailleurs, Lévesque et Lavoie (2007) ont travaillé sur les différences entre les sexes au niveau académique. Ils ont constaté que les garçons sont plus nombreux à être en difficulté d'apprentissage que les filles et sont plus en retard que celles-ci. De plus, il y a un écart de 4,9\% en faveur des filles pour ce qui est des résultats moyens obtenus aux évaluations d'écriture. Ces éléments vont dans le sens des résultats obtenus dans le cadre de cette étude à l'effet que les garçons font davantage d'erreurs de français que les filles, que ces erreurs soient liées au clavardage ou non.

Le lien entre le temps hebdomadaire consacré au clavardage et le sexe des sujets a également été vérifié, afin de constater si l'un des deux sexes clavarde davantage. Les résultats au test $t$ montrent à cette fin que les filles clavardent significativement plus que les garçons. Tous ces éléments vont ainsi dans le sens des résultats obtenus. Donc, les garçons font plus d'erreurs de français associées au clavardage que les filles, alors que celles-ci clavardent davantage que leurs camarades de classe du sexe opposé, et ce, depuis plus longtemps qu'eux, ce qui appuie les résultats reliés à la vérification de l'hypothèse principale, à l'effet que finalement, le clavardage n'a pas d'incidence défavorable sur la maîtrise du français écrit.

En résumé, concernant les différences entre les sexes quant à l'utilisation du clavardage, il est à noter que les filles et les garçons ont débuté à clavarder au même âge, que les filles clavardent significativement plus que les garçons, que ces derniers commettent plus d'erreurs de français associées au clavardage, malgré qu'il n'y ait pas de différence significative entre les garçons et les filles pour ce qui fait du fait qu'ils clavardent ou non.

La conclusion, qui fait suite à ce chapitre, permettra de clore ce mémoire de maittrise. 


\section{CHAPITRE 6 \\ CONCLUSION GÉNÉRALE}

\subsection{INTRODUCTION}

Dans cette partie, qui vise à conclure ce mémoire de maîtrise, seront rappelées les grandes lignes de celui-ci, dont l'objectif est de vérifier l'influence du clavardage sur la maîtrise du français écrit des élèves du premier cycle du secondaire. Les limites, les implications, de même que des avenues de recherches concernant le sujet à l'étude seront par la suite abordées.

\subsection{Problematique}

Le clavardage, qui se caractérise comme une activité permettant à un internaute de discuter virtuellement de façon instantanée et en temps réel avec d'autres internautes, est une forme de communication très populaire au Québec et ailleurs dans le monde. En effet, $74 \%$ des adolescents utilisent le clavardage pour entrer en communication avec autrui (Bouffard et Pouliot, 2007). De même, la génération caractérisant les adolescents d'aujourd'hui est une génération Internet, étant donné que ces jeunes passent beaucoup de temps à parcourir de diverses façons le monde virtuel. Internet fait donc partie intégrante de leur quotidien.

Des explications concernant les points positifs et négatifs du clavardage ont également été élaborées. Notamment, le clavardage facilite l'interaction des individus entre eux, de même que la prise de contact avec des gens du monde entier. Par ailleurs, des 
projets de clavardage pédagogique voient de plus en plus le jour dans les institutions scolaires, autant au Québec qu'ailleurs dans le monde. L'un des principaux points négatifs du clavardage est sans contredit la cyberintimidation, qui constitue réellement un problème de taille.

Lorsque nous analysons le phénomène à l'étude, il est primordial d'expliquer les formes linguistiques y étant associées, c'est-à-dire la façon dont les clavardeurs écrivent lorsqu'ils communiquent par le biais de ce média. Ces explications sur la langue codée sont aussi liées aux textos. Principalement, les internautes font appel à des réductions graphiques, c'est-à-dire qu'ils vont abréger des lettres et des mots. Les réductions avec variantes phonétiques sont aussi courantes. Ces réductions font en sorte que les gens modifient l'écriture des mots; l'important est alors que la prononciation reste la même pour que le message demeure compréhensible. Également, nous pouvons constater que certaines consonnes sont tout simplement coupées et que des mots sont parfois remplacés par une seule lettre. Encore ici, l'essentiel est que la prononciation de la lettre corresponde au son du mot. Une autre caractéristique du clavardage est les étirements graphiques, qui consistent à écrire plusieurs fois l'une des lettres du mot pour mettre l'emphase sur celui-ci. De même, le clavardage se caractérise comme étant une langue hétérogène, polyvalente, variable et où des troncations sont facilement visibles.

D'ailleurs, la langue utilisée pour clavarder possède plusieurs similitudes avec la langue orale. En effet, les internautes clavardent souvent comme ils parlent et certaines conditions de productions liées au clavardage possèdent plus de ressemblances avec l'oral qu'avec l'écrit.

Certes, les opinions concernant l'effet du clavardage sur la maîtrise de la langue sont mitigées. À vrai dire, pour certains, l'avènement de la langue utilisée par la communauté et par les adolescents pour clavarder n'est pas inquiétant pour ce qui est de la sauvegarde de la langue. À l'encontre de ces derniers, certaines personnes sont davantage inquiètes et estiment qu'il est possible que le clavardage ait un effet défavorable sur la maîtrise de la langue française. 
Peu d'études se sont jusqu'à maintenant attardées au phénomène du clavardage. Tout d'abord, Varnhagen et al. (2009) ont réalisé une recherche visant à déterminer si le clavardage a un effet sur la langue anglaise dans un contexte d'épellation. Les résultats ont démontré que le clavardage n'a pas d'influence sur la langue anglaise. Du côté francophone, une étude empirique cherchant à étudier l'influence du clavardage sur le français écrit a été réalisée par Lafontaine et al. (2005). Les résultats ont déterminé que le clavardage n'a pas d'influence négative sur le français écrit des jeunes de cinquième secondaire. David et Goncalves (2007), en ce qui les concerne, précisent que les jeunes français ne semblent pas confondre les deux langues (langue conventionnelle et langue codée) lors de travaux scolaires écrits.

De plus, Smith (1990) et Blake (2009) ont découvert l'influence positive du clavardage sur l'oral lors de l'apprentissage d'une langue seconde par le biais de deux études distinctes. Dans le cas de Smith, la langue seconde était l'espagnol, alors que pour Blake il s'agissait de l'anglais.

En somme, il importe de rappeler que plus d'opinions que d'études ont tenté d'expliquer le phénomène de l'influence du clavardage sur la maîtrise du français écrit. D'autre part, les recherches sur le clavardage ont davantage étudié l'incidence du clavardage sur l'écrit et sur l'oralité d'autres langues que le français, particulièrement l'anglais et l'espagnol. Une seule recherche (Lafontaine et al., 2005) a vérifié l'incidence du clavardage sur la maîtrise du français écrit des élèves francophones québécois et elle a révélé que celui-ci n'affecte pas négativement la langue écrite française. Nous pouvons facilement constater que cette étude possède plusieurs similitudes avec la nôtre. Cependant, il importe de préciser qu'il s'agit de la seule étude qui a étudié l'impact du clavardage sur le français écrit au Québec, ce qui ne suffit pas pour le domaine éducatif. De plus, les sujets y ayant participé sont des élèves de cinquième secondaire, ce qui fait en sorte que la clientèle ciblée n'est pas la même que pour les besoins de ce mémoire. Les élèves de cinquième secondaire sont plus avancés dans leur apprentissage du français que les élèves de secondaires un et deux, qui constituent les sujets de la présente étude; ils ont davantage eu 
l'occasion de rédiger des textes et d'apprendre les normes de la langue française. De ce point de vue, il est possible que les caractéristiques de l'échantillon aient une influence sur les résultats finaux. D'ailleurs, des variables différentes de celles attribuées à l'étude de Lafontaine $e t$ al. ont été prises en considération dans le cadre de ce mémoire. Notamment, l'âge de début du clavardage n'a jamais été considéré lors d'une étude sur le clavardage, de même que le temps hebdomadaire y étant consacré. D'après nous, ces dernières variables sont essentielles dans la démarche de compréhension du phénomène. Également, la distinction entre les sexes, cela pour chaque variable en statistiques descriptives, amène de nouvelles données concernant les habitudes de clavardage des adolescents d'aujourd'hui. Ainsi, plusieurs éléments associés à notre recherche sont tout à fait nouveaux pour le monde éducatif. Tout compte fait, peu est connu sur ce phénomène d'actualité qui doit être davantage documenté, ce qui nous mène vers les explications du cadre théorique, dans l'objectif d'apporter des éléments de réponse à la question suivante : quelle est l'influence du clavardage sur la maîtrise du français écrit des élèves du premier cycle du secondaire?

\subsection{CADRE THEORIQUE}

Le cadre théorique, dont les bases sont centrées sur l'influence de la psychologie cognitive sur l'apprentissage, a permis de faire ressortir les points principaux associés à cette forme de psychologie en lien avec le clavardage. Certains éléments laissent entendre que le clavardage n'est pas inquiétant pour ce qui est de l'évolution de la langue française. Toutefois, d'autres points semblent indiquer le contraire, c'est-à-dire que l'avènement du clavardage doit être considéré d'un œil craintif.

En premier lieu, la métacognition doit être prise en compte selon deux aspects bien distincts. D'une part, ce concept met en évidence le fait que les jeunes qui possèdent de bonnes aptitudes métacognitives devraient être capables de distinguer quand ils doivent faire appel à chaque forme de langue, soit la langue codée et le français conventionnel. Par contre, il est nécessaire de se questionner concernant les élèves en diffịculté 
d'apprentissage. En effet, il est possible que ces élèves aient davantage de difficulté à faire la part des choses et soient ainsi susceptibles de transférer les mots codés du clavardage dans leurs écrits scolaires, où ils doivent utiliser un français conventionnel. Ces élèves ont au départ des lacunes aux niveaux cognitifs et métacognitifs.

De plus, les jeunes ont développé au fil de leurs nombreuses heures de clavardage un nouveau répertoire de mots et d'expressions, qu'ils ont fini par mémoriser, pour en venir ensuite à les automatiser. En effet, plus ils passent de temps à clavarder, plus ils finissent par automatiser cette forme d'écriture. L'apprentissage de la langue codée semble donc se cristalliser de cette manière, par le biais de la pratique. Suite à cette affirmation, nous pouvons supposer que plus les élèves ont d'heures de clavardage à leur actif, plus ils sont à risque de transférer la langue codée dans leurs écrits scolaires. Malgré cela, nous pouvons également penser que les jeunes d'aujourd'hui ont dans leur système cognitif deux représentations du même mot, soit une représentation pour le clavardage et une seconde pour les autres contextes, particulièrement les contextes scolaires et professionnels. Selon ce dernier élément pris en considération, les jeunes pourraient être capables de cerner quand ils doivent recourir à chaque forme de français, sauf peut-être les élèves en difficulté d'apprentissage tel que mentionné précédemment.

Qui plus est, nous savons que les jeunes ne transfèrent pas le français conventionnel dans leurs conversations virtuelles, mis à part pour certains mots, bien entendu. Alors, ils devraient logiquement agir de la même façon lorsqu'ils rédigent un texte en classe. Effectivement, ils écrivent volontairement les mots de la mauvaise façon lorsqu'ils clavardent. En situation scolaire, ils peuvent aussi décider d'écrire leur texte en usant des mots respectant les balises de la langue française et ainsi démontrer une bonne maîtrise de la langue.

La question des variations linguistiques doit être considérée. Effectivement, les personnes recourant à la langue codée en contexte de clavardage ont tendance à modifier la langue de différentes façons, de sorte qu'ils en font un type de variation linguistique. Nous pouvons également lier le clavardage au verlan, qui consiste en une variation linguistique 
de la langue orale française utilisée par certains adolescents. En France, Bertucci (2003) mentionne que les jeunes parlent souvent verlan, qui est une langue orale qui se démarque par sa vulgarité et son côté obscène. Ils utilisent cette langue lorsqu'ils sont avec leurs amis, mais conservent une langue orale adéquate lorsqu'ils sont en classe. En revanche, pour les jeunes ayant des troubles d'apprentissage, ceux-ci ont davantage de difficulté à discerner quand ils peuvent parler verlan et quand ils ne le peuvent pas, surtout en contexte formel, tel que le précise l'auteure. Nous pouvons ici faire le parallèle avec le verlan et le clavardage. En effet, les jeunes en difficulté d'apprentissage, qui ont généralement des aptitudes métacognitives moins développées que les élèves plus forts, pourraient être plus à risque de transférer la langue codée du clavardage dans leurs écrits scolaires.

Enfin, nous savons que les jeunes qui clavardent omettent d'utiliser des stratégies d'écriture lorsqu'ils discutent par le biais du clavardage. Puisqu'ils y sont habitués, il est légitime de se demander s'ils automatiseront la non-utilisation de ces stratégies, ce qui les amènerait progressivement à ne plus utiliser de stratégies d'écriture en processus de rédaction à l'école. À cet égard, les particularités des élèves en difficulté doivent encore une fois être prises en compte. En effet, contrairement aux élèves plus forts, ceux-ci doivent prendre le temps de réfléchir aux stratégies d'écriture requises lorsqu'ils écrivent un texte puisque souvent leur utilisation n'a pas été automatisée. S'ils ont en plus pris l'habitude de ne plus y recourir en clavardant, il est possible qu'ils agissent de la même manière à l'école, sans y réfléchir.

En somme, suite à l'analyse des écrits recensés pour ce cadre théorique, plusieurs questions se sont posées. Certains éléments mentionnés tendent vers une possible influence du clavardage sur la maîtrise de la langue. Notamment, il est nécessaire de prendre en compte les particularités des élèves en difficulté d'apprentissage, qui sont pour plusieurs d'entre eux intégrés dans des groupes réguliers. Ces élèves, compte tenu de leurs lacunes au niveau métacognitif, constituent une clientèle à risque, ce qui contribue au fait que l'on pourrait voir des traces de la langue codée dans les écrits scolaires. Ainsi, l'hypothèse 
principale se pose comme suit : le clavardage a une influence défavorable sur la maitrise du français écrit des élèves du premier cycle du secondaire.

\subsection{RECHERCHE}

D'abord, il est important de rappeler les caractéristiques de l'échantillon. Ainsi, 158 élèves du premier cycle du secondaire régulier, donc de secondaire un et deux, ont participé à la recherche. Ces jeunes, soit 90 garçons et 68 filles, sont âgés entre 12 et 16 ans.

Chez ces sujets, les quatre variables suivantes ont été mesurées : l'âge de début du clavardage, le temps hebdomadaire consacré au clavardage, le fait que l'élève clavarde ou non et les erreurs de français associées au clavardage. Des hypothèses liées à chaque variable ont été formulées et vérifiées.

Tel que spécifié, l'hypothèse principale, qui met de l'avant que le clavardage a une influence défavorable sur la maîtrise du français écrit des élèves du premier cycle du secondaire, a été testée de différentes façons. Premièrement, il a été vérifié si les élèves qui ont commencé à clavarder à un plus jeune âge ont effectué plus d'erreurs de français associées au clavardage que les adolescents ayant commencé à le faire à un âge plus tardif. Il en ressort que les élèves qui ont commencé à clavarder très jeunes n'ont pas nécessairement fait plus d'erreurs de français associées au clavardage que ceux ayant débuté à le faire plus tard.

Suite à cette analyse, l'hypothèse suivante a été vérifiée : plus les élèves clavardent durant la semaine, plus ils sont susceptibles de faire des erreurs de français associées au clavardage. Les résultats ont montré que le temps passé à clavarder n'a pas d'incidence non plus sur le nombre d'erreurs de français associées au clavardage.

Pour ce qui est de la variable le fait que l'élève clavarde ou non, il a été vérifié si le clavardage en tant que tel, par rapport aux clavardeurs et aux non-clavardeurs, a une incidence sur le nombre d'erreurs. Les résultats ont démontré qu'il n'y a pas de différence 
significative entre les élèves qui clavardent et ceux qui ne s'y prêtent pas du tout, toujours par rapport aux erreurs de français associées au clavardage.

Donc, les trois hypothèses secondaires visant la vérification de l'hypothèse principale n'ont pas été confirmées, ce qui indique que le clavardage n'a pas d'influence défavorable sur la maîtrise du français écrit des élèves du premier cycle du secondaire. Les résultats à ces trois hypothèses sont donc concordants entre eux.

D'ailleurs, d'autres études ont abouti à des résultats semblables. Notamment, l'étude de Lafontaine et al. (2005) est arrivée aux mêmes constats, c'est-à-dire qu'elle a conclu, à la suite d'évaluations écrites, que le clavardage n'a pas d'incidence sur le français écrit des élèves de cinquième secondaire. De plus, l'étude anglophone de Varnhagen et al. (2009) a déterminé que le clavardage n'influence pas la langue anglaise des adolescents en situation d'épellation. En ce qui nous concerne, nous avions une opinion mitigée au départ quant à l'influence du clavardage sur le français, ce qui nous a poussées à travailler sur ce sujet. Les lectures réalisées concernant le clavardage, de même que les différentes recherches, nous ont aussi amenées à nous questionner davantage. Nous sommes néanmoins heureuses de constater que l'avènement du clavardage ne nuit pas à la maîtrise de la langue française écrite.

\subsection{Limites}

Comme dans toute recherche, celle-ci comporte des limites qui se doivent d'être soulevées. D'une part, il importe de rappeler que l'expérimentation s'est déroulée avec des élèves se situant dans des groupes réguliers. Des groupes plus forts et d'adaptation scolaire n'ont donc pas participé au projet. En revanche, de nos jours, il est fréquent que des élèves ayant des difficultés d'apprentissage se retrouvent malgré tout dans des groupes réguliers. Cet élément fait en sorte que plusieurs dictées ont sans doute été écrites par des élèves en difficulté. Nous pouvons ainsi affirmer que ces élèves n'ont pas été mis à l'écart puisqu'ils font probablement partie de l'ensemble des sujets. C'est pourquoi les particularités de ces 
élèves au niveau métacognitif ont été abordées dans le cadre théorique. Toutefois, nous ne pouvons pas dénombrer ces élèves puisqu'il n'était pas prévi dans le cadre de cette recherche de recueillir des informations les concernant spécialement. Il s'agit d'ailleurs d'une limite de cette étude. Il demeure qu'il serait intéressant pour le domaine éducatif d'étudier plus en profondeur l'influence du clavardage sur la maîtrise du français écrit de ces élèves en particulier. En effet, plusieurs d'entre eux ont au départ de la difficulté à rédiger un texte et commettent plus d'erreurs d'écriture que les élèves plus forts. Nous pouvons donc nous questionner sur leur aptitude à ne pas transférer les écrits du clavardage dans leurs écrits scolaires, surtout s'ils passent de nombreuses heures par semaine à clavarder.

D'autre part, les différentes étapes de la recherche ont eu lieu dans une seule et même polyvalente du Bas-St-Laurent. Il s'agit d'une école où les élèves complètent les cinq années de leur secondaire. Il est également important de mentionner que l'école se situe dans un milieu défavorisé, ce qui a pu peut-être jouer un rôle sur les résultats. Il aurait aussi été pertinent de réaliser l'expérimentation dans une ou deux autres écoles dans le but d'obtenir des résultats plus complets et de vérifier s'ils auraient été semblables. De même, il aurait été intéressant d'aller expérimenter dans d'autres régions du Québec, par exemple dans les grands centres tels que Québec et Montréal, ou les petits villages en région. Les résultats obtenus dans le cadre de ces expérimentations auraient peut-être été différents. Par contre, il suffit de penser à l'étude de Lafontaine et al. (2005), qui s'est déroulée dans la région de Québec. En effet, cette recherche a montré elle aussi que le clavardage n'a pas d'influence défavorable sur le français écrit des jeunes d'aujourd'hui, ce qui nous ramène aux résultats de notre propre recherche.

Concernant la correction des dictées, il est certain qu'il a été difficile de déterminer quelles erreurs devaient être comptabilisées comme étant attribuables au clavardage. Pour ce faire, nous nous sommes basées sur nos observations sur les sites de clavardage, sur les commentaires des jeunes à propos de leur style d'écriture lorsqu'ils clavardent, de même que sur les caractéristiques du clavardage rapportées par les écrits. Les éléments 
mentionnés concernant les erreurs demeurent subjectifs puisque lors de la correction, il est impossible de connaître l'état d'esprit de l'élève et si, par exemple, il enlève un s à la fin d'un mot parce qu'il ne sait pas comment l'orthographier ou parce qu'il l'écrit de cette façon lorsqu'il clavarde. Il demeure qu'un répertoire des erreurs considérées comme telles figure en annexe II de ce mémoire et que toutes les erreurs citées ont été prises en considération lors de la correction des dictées, cela pour chaque élève.

Pour ce qui est du questionnaire, les élèves ont eu tout le temps nécessaire pour y répondre. Toutefois, il est possible que certains élèves, dans le but de terminer plus rapidement, aient répondu aux questions sans réellement s'y attarder. Par exemple, lorsqu'ils devaient inscrire le nombre d'heures consacrées au clavardage par semaine, il est impossible de savoir si l'élève a pris la peine de bien calculer, s'il a inscrit à peu près le chiffre exact ou s'il a écrit un nombre aléatoire pour se débarrasser de la tâche. Cet aspect peut donc affecter les données étant donné que le temps hebdomadaire consacré au clavardage est une variable importante de la recherche.

Il aurait aussi été intéressant de vérifier les résultats en français des sujets; cette vérification aurait permis de mieux situer leurs performances scolaires en écriture et de constater s'il y a un lien entre le nombre d'erreurs de la dictée et les résultats scolaires du bulletin. Il aurait alors été possible de vérifier si les élèves dont les résultats scolaires mettent en évidence leurs difficultés d'apprentissage ont employé davantage la langue codée lors de la rédaction de la dictée. Nous aurions alors pu catégoriser les sujets selon les informations fournies par les enseignants et ainsi, enrichir les résultats. En somme, il est possible que les éléments susmentionnés aient eu une influence sur l'interprétation des résultats de la recherche. 


\subsection{IMPLICATIONS}

Abordons maintenant les implications éducatives, sociales et scientifiques qu'apportent les résultats de ce mémoire de maîtrise. Des avenues de futures recherches seront aussi proposées.

\subsubsection{Implications éducatives}

Au niveau éducatif, il est certain que cette recherche permettra aux enseignants de mieux cerner les particularités de la langue codée associée au clavardage, de même que les écritures y correspondant. En effet, ils y auront accès par le biais de cette recherche, autant par les explications concernant les caractéristiques de la langue codée que dans la liste des erreurs de français associées au clavardage commises par les élèves, qui est placée en annexe II. Il sera donc plus facile pour eux de dépister les mots qui semblent issus de cette langue dans les productions écrites de leurs élèves. Néanmoins, l'analyse des résultats démontre qu'en fin de compte, le nombre de mots codés dans les écrits scolaires des jeunes n'est pas inquiétant, compte tenu qu'il n'est pas trop élevé.

Également, cette recherche pourrait conduire vers de nouvelles avenues concernant les projets de clavardage pédagogique. Effectivement, les enseignants savent maintenant que le clavardage n'est pas nocif pour la maîtrise de la langue écrite conventionnelle, ce qui fait en sorte qu'il n'y a rien à craindre à centrer une partie de leur enseignement sur cette méthode pédagogique qui, de par son originalité, devrait plaire aux jeunes de plusieurs groupes d'âge. Comme de fait, les adolescents d'aujourd'hui, qui font partie de la génération Internet, se démarquent justement par leur appréciation du monde virtuel et du clavardage, d'autant plus qu'ils y ont souvent recours. De plus, il s'agit d'une formule pédagogique qui pourrait accroître la motivation des élèves, étant donné qu'ils apprécient généralement ce média. De même, le clavardage a l'avantage d'inciter les jeunes à écrire. Par exemple, les enseignants pourraient proposer des activités de clavardage pédagogique, 
mais requérant l'utilisation correcte du français conventionnel. Réaliser une recherche sur le potentiel du clavardage pédagogique s'avèrerait donc particulièrement pertinent.

D'autre part, la recension des écrits a permis de constater que le clavardage améliore les performances orales des adolescents lors de l'apprentissage d'une langue seconde. Nous pouvons donc supposer que ce média pourrait agir de la même façon chez les élèves

québécois francophones et donc, que les performances des jeunes lors des évaluations orales dans leurs cours d'anglais langue seconde subiraient une progression. Effectivement, les élèves, par le biais du clavardage, pourraient enrichir leur vocabulaire et mettre en application l'apprentissage de la syntaxe inhérente à cette langue seconde. Ils pourraient alors transférer ces apprentissages à la langue orale.

Pour terminer, les enseignants savent maintenant que ce moyen de communication n'a pas d'influence défavorable sur la maittrise de la langue écrite, ce qui fait en sorte qu'il n'est plus nécessaire pour eux de s'inquiéter concernant l'essor de ce phénomène, qui demeurait préoccupant pour certains enseignants en activité. Il demeure qu'il serait pertinent de prévoir davantage d'activités d'écriture, ce qui serait profitable pour les élèves se situant dans des groupes réguliers, mais encore plus pour ceux vivant avec des difficultés d'apprentissage, cela pour que le temps de clavardage ne dépasse pas le temps consacré à l'écriture conventionnelle. Les enseignants ont aussi une tâche de prévention à accomplir; ils doivent avertir leurs élèves qu'ils doivent garder à l'esprit quelle écriture ils doivent employer lorsqu'ils écrivent un texte, dans le but d'être certain que ce point demeure actif au niveau métacognitif.

\subsubsection{Implications sociales}

Pour ce qui est des implications sociales, il est intéressant pour les parents de savoir qu'il n'est pas nécessaire de s'alarmer lorsqu'ils constatent un nombre élevé d'erreurs de français visibles sur l'écran d'ordinateur de leurs enfants. Cette recherche permettra justement aux parents de savoir qu'il s'agit d'une langue codée qui s'utilise uniquement en 
contexte de clavardage, comme une langue seconde, et que les jeunes d'aujourd'hui sont capables de discerner les différences entre les deux langues et ainsi savoir quand ils peuvent y recourir. Pour les parents comme pour les enseignants et autres acteurs du monde de l'éducation, il s'agit d'une préoccupation en moins. Toutefois, compte tenu des limites de la recherche énoncées précédemment, les résultats associés à cette étude peuvent différer selon plusieurs caractéristiques, dont les caractéristiques personnelles et académiques des élèves (forces et faiblesses), la région où s'est déroulée la cueillette de données, l'enseignement préconisé par l'enseignant...De plus, ces résultats ne peuvent pas nécessairement être adaptés envers les élèves en difficulté d'apprentissage compte tenu de leurs spécificités; il n'est donc pas possible de généraliser ces résultats à tous les élèves. Cette recherche permettra également d'enrichir les statistiques descriptives concernant le clavardage, qui étaient peu nombreuses au Québec. À notre connaissance, aucune recherche n'a fait mention jusqu'à aujourd'hui de l'âge lors duquel les jeunes débutent en moyenne à clavarder, de même que le temps hebdomadaire moyen consacré au clavardage, autant selon les jeunes en général que selon les sexes. Ce mémoire apporte donc une nouvelle source de données concernant ce média très populaire, mais encore sous-étudié. D'autre part, l'ensemble de la société peut maintenant savoir qu'une étude rigoureuse a vérifié que le temps passé à clavarder chaque semaine n'a pas d'influence sur les erreurs de français associées au clavardage, étant donné qu'elles ne sont pas davantage nombreuses chez les élèves qui clavardent beaucoup.

À l'opposé, il importe de se rappeler un côté plus négatif relié au clavardage, de même qu'aux médias sociaux. Il s'agit de la cyberintimidation, qui est de plus en plus présente sur Internet. Même si ce mémoire ne traite pas de cyberintimidation, il est nécessaire de mentionner ce phénomène puisqu'il constitue un véritable problème. Les parents, autant que les enseignants et les autres personnes impliquées, doivent agir, prévenir et surveiller les jeunes quant à la possible influence de la cyberintimidation sur le cheminement personnel et scolaire des adolescents. Cette forme d'intimidation peut affecter les jeunes à plusieurs niveaux et faire de leur scolarité une période très difficile. Il est donc nécessaire de faire de la prévention quant à ce phénomène qui prend de plus en plus 
d'ampleur. Notamment, l'école pourrait réaliser des activités reliées à l'anticyberintimidation et aborder avec les enseignants, les parents et les élèves la gravité du harcèlement par le biais d'Internet et des téléphones cellulaires. Les parents ont également un rôle à jouer. Par exemple, ceux-ci devraient faire preuve de vigilance, s'impliquer dans la vie virtuelle de leurs adolescents et leur faire savoir qu'ils désirent être au courant s'ils vivent une situation de cyberintimidation (Réseau Éducation-Médias, 2009). Pour ce qui est des enseignants, ils doivent garder leurs jeunes à l'œil s'ils planifient et réalisent des projets de clavardage pédagogique, pour être certains que les conversations se limitent au cadre scolaire.

\subsubsection{Implications scientifiques et avenues de recherches}

Cette recherche permettra aux chercheurs actuels et futurs d'avoir accès à une étude répondant à plusieurs questions. En effet, les résultats obtenus dans le cadre de ce mémoire ont apporté diverses réponses qui pourraient être encore davantage précisées par l'intermédiaire de recherches complémentaires. Plusieurs pistes de recherches devraient être envisagées dans un avenir rapproché.

Il serait ainsi de circonstance de s'attarder au phénomène du clavardage par rapport aux élèves se situant dans des groupes d'adaptation scolaire. Il serait alors possible de vérifier si ces élèves transfèrent davantage la langue codée du clavardage dans leurs écrits scolaires, comparativement aux élèves des groupes réguliers. En effet, les écrits recensés dans le cadre théorique mettent de l'avant le fait que les élèves en difficulté ont des habiletés métacognitives moins développées que les élèves plus forts, ce qui pourrait peutêtre avoir un impact sur le fait qu'ils transféreront ou non la langue codée attribuée au clavardage dans leurs écrits scolaires. Il serait aussi bien pensé d'étudier plus en profondeur les côtés cognitifs et métacognitifs des élèves en difficulté, toujours concernant le phénomène de la présente recherche. De même, réaliser une recherche sur le clavardage tout en comparant entre eux différents milieux serait également pertinent. À cette fin, les chercheurs pourraient comparer le nombre d'erreurs de français associées au clavardage 
selon les milieux défavorisés et les milieux plus aisés, ainsi que les résultats des grands centres urbains à ceux des régions. De surcroît, des chercheurs pourraient également vérifier l'influence du clavardage sur la maîtrise du français écrit sur une échéance à plus long terme, à savoir si les heures cumulées finiront par avoir une incidence sur le français, au fil des années. Le clavardage pourrait donc être étudié selon une perspective longitudinale. En effet, ces élèves auraient automatisé sur une plus longue période la langue associée au clavardage que la clientèle ciblée dans ce mémoire. De plus, puisque les élèves du primaire débutent pour certains d'entre eux à clavarder en même temps qu'ils apprennent les rudiments de l'écriture, une recherche centrée sur leur réalité serait tout aussi intéressante et pourrait peut-être déboucher sur d'autres constats. En effet, ces jeunes enfants n'auraient pas eu l'occasion d'apprendre séparément le français conventionnel et la langue codée, comme les adolescents actuels du secondaire; ils auraient appris les deux langues d'une manière successive. Enfin, dans le cadre de cette recherche, nous avons abordé le clavardage pédagogique. Il serait pertinent d'étudier ce moyen pédagogique plus en profondeur, afin d'établir les bienfaits qu'il pourrait peut-être apporter chez différentes clientèles de jeunes, particulièrement au niveau des jeunes ayant des difficultés d'écriture et des troubles de comportement. Il demeure que le clavardage pédagogique pourrait accroître la motivation de ces jeunes envers l'apprentissage. 


\section{ANNEXE I}

\section{DICTÉE}

\section{Une discussion entre amies}

- Salut Caroline. Qu'est-ce que tu fais aujourd'hui?

- Je m'en vais chez Julie.

- Vous allez faire quoi?

- On va sûrement écouter un film ou aller au cinéma. On n'a pas encore décidé, parce que nous n'avons pas encore réussi à nous voir.

- Avez-vous une idée de ce que vous allez écouter? Quels sont les nouveaux films?

- Je ne sais pas. Je n'ai même pas regardé.

- En tout cas, c'est une bonne idée! Je pensais justement y aller en fin de semaine. Mais je n'ai pas encore eu le temps.

- Pourquoi?

- Je suis très occupée ces jours-ci.

- À part ça, quoi de neuf?

- Je me suis fait mal à la jambe. Je suis tombée en vélo hier.

- Comment c'est arrivé?

- Je suis arrivée sur une bosse qui a fait renverser mon vélo.

- Dis-moi, est-ce que tu as su que l'enseignante d'anglais est en congé de maternité?

- Non, je ne le savais pas.

- Ça va sûrement être moins tranquille dans les cours. Tout le monde va s'en permettre. En plus, il commence à faire beau dehors.

- Je sais. Moi, j'ai décidé que j'avais envie de réussir mes cours et de passer mon année. Jusqu'à ce que je sois tannée, j'imagine. J'ai beaucoup de retard à rattraper. 



\section{ANNEXE II}

\section{REGISTRE DES ERREURS}

Étant donné les particularités de la langue codée utilisée lors de sessions de clavardage, voici une liste de toutes les erreurs qui ont été relevées lors de la correction des

dictées pour la variable erreurs de français associées au clavardage. Il s'agit autant des mots cités lors de la dictée que ceux écrits par les élèves lorsqu'ils devaient rédiger les cinq lignes supplémentaires de leur propre gré. Tous ces mots et ces groupes de mots ont donc été placés dans cette liste, afin de créer un registre des erreurs qui ont été comptabilisées lors de la correction. Dans la colonne de gauche figurent les mots et les groupes de mots dans leur forme correcte, tandis que dans la colonne de droite, vis-à-vis ces derniers, sont indiqués les mêmes mots et groupes de mots s'apparentant à la langue codée tels que décelés dans la dictée et dans la petite rédaction. Plusieurs erreurs peuvent être attribuées à un même mot ou groupe de mots, ce qui fait en sorte que toutes les déclinaisons possibles en sont indiquées dans la colonne de droite. Ces mots et groupes de mots sont classés par ordre alphabétique. Le tableau 6 présente les mots erronés, alors que le tableau 7 présente les groupes de mots où des erreurs ont été ciblées. 
Tableau 6 : Mots inclus dans le registre des erreurs

\begin{tabular}{|c|c|}
\hline Mots bien orthographiés & Mots en langue codée \\
\hline 1) alors & 1) alor \\
\hline 2) allons & 2) alon \\
\hline 3) année & 3) anné \\
\hline 4) bien & 4) ben \\
\hline 5) classe & 5) class \\
\hline 6) commence & 6) comence \\
\hline 7) comment & 7) commen \\
\hline 8) concours & 8) concour \\
\hline 9) d'accord & 9) dacore \\
\hline 10) dehors & 10) dehor \\
\hline 11) difficile & 11) dificil \\
\hline 12) effort & 12) effor \\
\hline 13) encore & 13) encor ; ancor \\
\hline 14) en tout cas & 14) entoutca; en tk \\
\hline 15) envie & 15) envi \\
\hline 16) faire & 16) fair \\
\hline 17) idée & 17) idea; idé \\
\hline 18) instant & 18) instan \\
\hline 19) justement & 19) justemen \\
\hline 20) mais & 20) mai \\
\hline 21) m'appelle & 21) m’appel \\
\hline
\end{tabular}




\begin{tabular}{|c|c|}
\hline Mots bien orthographiés & Mots en langue codée \\
\hline 22) moins & 22) moin \\
\hline 23) ok & 23) oki \\
\hline 24) pourquoi & 24) pkoi ; pourkoi \\
\hline 25) professeur & 25) prof \\
\hline 26) quoi & 26) koi ; coi \\
\hline 27) retard & 27) retar \\
\hline 28) samedi & 28) samdie \\
\hline 29) semaine & 29) semain \\
\hline 30) sûrement & 30) surment \\
\hline 31) tantôt & 31) tanto \\
\hline 32) temps & 32) temp \\
\hline 33) toujours & 33) toujour \\
\hline 34) tranquille & 34) tranquil ; trankil \\
\hline 35) mot anglophone & 35) cool \\
\hline 36) mot anglophone & 36) bike \\
\hline 37) mot anglophone & 37) hot \\
\hline 38) mot anglophone & 38) fun \\
\hline 39) mot inventé & 39) tchiler \\
\hline 40) petit sourire (emoticons) & 40) : ) \\
\hline
\end{tabular}


Tableau 7 : Groupes de mots inclus dans le registre des erreurs

\begin{tabular}{|c|c|}
\hline $\begin{array}{l}\text { Groupe de mots } \\
\text { bien orthographiés }\end{array}$ & Groupe de mots codés \\
\hline 1) aimes-tu ça & 1) aime tu sa \\
\hline 2) à la prochaine & 2) à la prochain \\
\hline 3) à part & 3) a par \\
\hline 4) à part ça & 4) a par sa \\
\hline 5) à plus & 5) à + \\
\hline 6) bon bien & 6) bon ben \\
\hline 7) ça me fait plaisir & 7) sa me fait plaisir \\
\hline 8) ça va & 8) sa va \\
\hline 9) ça va être & 9) sa va être \\
\hline 10) ce soir & 10) se soir \\
\hline 11) ces jours-ci & 11) ses jours ci ; ses jour ci \\
\hline 12) c'est amusant & 12) ces le fun \\
\hline 13) c'est mon père & 13) ses mon père \\
\hline 14) c'est possible & 14) ces possible \\
\hline 15) c'est pour ça & 15) ses pour sa \\
\hline 16) c'est une bonne idée & 16) ces une bonne idée \\
\hline 17) c'est vrai & 17) ses vrai \\
\hline 18) comment c'est arrivé & $\begin{array}{l}\text { 18) comment cé arrivé; comment ces } \\
\text { arrivé }\end{array}$ \\
\hline 19) de ce que & 19) de se que \\
\hline
\end{tabular}




\begin{tabular}{|c|c|}
\hline $\begin{array}{l}\text { Groupe de mots } \\
\text { bien orthographiés }\end{array}$ & Groupe de mots codés \\
\hline 20) en tout cas & 20) entouka \\
\hline 21) est-ce que & 21) esceque; esseque \\
\hline 22) je déteste ça & 22) je déteste sa \\
\hline 23) je n’ai & 23) je n’é \\
\hline 24) je ne sais pas & 24) je ne ces pas; je ne ses pas \\
\hline 25) je te laisse sur ça & 25) je te laisse sur sa \\
\hline 26) jusqu'à ce que & 26) jusqu'à se que ; juscas-se que \\
\hline 27) j’y aille & 27) ji aille \\
\hline 28) moi aussi & 28) moi si \\
\hline 29) qu'est-ce que & 29) quesse que \\
\hline 30) qu'est-ce que tu vas faire & 30) ques tu va faire \\
\hline 31) quoi de neuf & 31) koi de neuf ; koi de 9 \\
\hline 32) si ça te dit & 32) si sa te dit \\
\hline 33) tu gardes ça & 33) tu garde sa \\
\hline 34) t'imagines-tu ça & 34) t'imagine tu sa \\
\hline 35) tu es & 35) tes \\
\hline 36) tu n'es pas chanceuse & 36) tes pas chanceuse \\
\hline 37) y aller & 37) i aller \\
\hline
\end{tabular}





\section{UQAR}

Université du Quebec

a Rimouski

Faculté des sciences de l'éducation

\section{ANNEXE III}

\section{QUESTIONNAIRE SUR LES HABITUDES INFORMATIQUES}

1) Possèdes-tu ton propre ordinateur?
a) Oui
b) Non

2) Si non, utilises-tu régulièrement l'ordinateur familial?
a) Oui
b) Non

3) Combien de temps par semaine, en moyenne, passes-tu devant l'ordinateur?

4) Tu vas surtout sur Internet pour (coche la réponse appropriée) :
a) clavarder («chatter »)
b) chercher des informations pour ta culture personnelle
c) télécharger de la musique
d) tes travaux scolaires

5) Mets les éléments de la question 4 en les notant par ordre d'importance sur les lignes à côté des énoncés (de 1 à 4), selon le temps que tu passes sur Internet. 
6) Combien de temps par semaine clavardes-tu en moyenne?

7) Si tu clavardes tous les jours, combien de temps par jour clavardes-tu en moyenne?

8) Décris en tes mots l'écriture que tu emploies lorsque tu clavardes avec tes amis:

\section{Merci beaucoup pour ton temps et ta collaboration !}




\section{RÉFÉRENCES BIBLIOGRAPHIQUES}

Anis, J. (2000). L'écrit des conversations électroniques de l'Internet. Le français aujourd'hui, 129, 59-69.

Anis, J. (2003). Communication électronique scripturale et formes langagières: chats et SMS. Actes des quatrièmes rencontres Réseaux Humains/Réseaux technologiques (pp. 57-70). Poitiers: Documents, Actes et Rapports pour l'éducation. Récupéré le 15 novembre 2009 de http ://rhrt.edel.univ-poitiers. fr/document.php?id=547

Bazin, A. et Girerd, R. (1997). La métacognition, une aide à la réussite des élèves du primaire. Dans M. Grangeat et P. Meirieu (Dir.), La métacognition, une aide au travail des élèves (pp. 63-93). Paris : ESF éditeur.

Bernier, M. (2007). Obstacles à la communication dans une situation de communication exolingue en contexte de clavardage. Actes de la $13^{e}$ Journée Sciences et Savoirs. Sudbury: Université Laurentienne, département d'études françaises et de traduction.

Bertucci, M. M. (2003). Les parlers jeunes en classe de français. Le français aujourd'hui, 143, 25-34.

Blake, C. (2009). Potential of Text-Based Internet Chats for Improving Oral Fluency in a Second Language. The Modern Language Journal, 93(2), 227-240.

Boudreau, G. (1995). Processus cognitifs des étudiants du postsecondaire en production de textes. Revue des sciences de l'éducation, XXI(1), 75-93.

Bouffard, M. et Pouliot, M. J. (2007). Diversité linguistique et Internet. Le cas de la messagerie instantanée et des forums de discussion sur Internet. Récupéré le 25 novembre 2009 du site Diversité Linguistique: http://wikini-backup. tuxcafe.org/wakka.php?wiki=DiversiteLinguistique

Bourdieu, P. (1983). Vous avez dit «populaire». Actes de la Recherche en Sciences

Sociales, 46, 98-105. 
Bourny, G., Braxmeyer, N., Dupé, C., Remond, M., Robin, I. et Rocher, T. (2002). Les compétences des élèves français à l'épreuve d'une évaluation internationale. Premiers résultats de l'enquête PISA 2000. France: Ministère de la Jeunesse, de l'Éducation nationale et de la Recherche, Direction de la programmation et du développement, sous-direction de l'évaluation.

Brown, A. (1987). Metacognition, Executive Control, Self-regulation and other more Mysterious Mechanisms. Dans E. E. Weinert et R. H. Kluwe (Dir.), Metacognition, motivation and understanding (pp. 65-116). Hillsdale, N.J.: Lawrence Erlbaum Associates.

Cadet, B. (1998). Psychologie cognitive. Paris : In Press.

Cerisier, J. F. (2003) Usages des technologies de la communication et maîtrise de la langue : l'œuf et la poule. Actes des Quatrièmes Rencontres Réseaux Humains / Réseaux technologiques (pp. 11-13). Poitiers: Actes et Rapports pour l'Éducation. Récupéré le 15 novembre 2009 de http://rhrt.edel.univpoitiers. fr/document. php?id=533

Chantelois, P. (2008). La cyberintimidation: "À ta place, je me suiciderais. » Récupéré le 15 janvier 2010 du site Cent-papiers: http://www.centpapiers.com/lacyberintimidation-a-ta-place-je-me-suiciderais/2302/

Chouinard, M. A. (2002). Échanges électroniques en direct: le chat et la souris. La communication par code du clavardage, très populaire chez les jeunes, menace-telle la qualité de la langue? Le Devoir. Récupéré le 13 novembre 2009 de http ://www.ledevoir.com/societe/education/9126/echanges-electroniques-endirect-le-chat-et-la-souris

Chouinard, R. (1998). Autorégulation, motivation et intervention auprès des élèves en difficulté. Dans P. Mongeau et L. Lafortune (Dir.), Interventions métacognitives : expérimentations et évaluation (pp. 101-129). Montréal : les Éditions Logiques.

Chouinard, R., Plouffe, C. et Roy, N. (2004). Caractéristiques motivationnelles de garçons du secondaire en difficulté d'apprentissage ou en trouble de la conduite. Revue des sciences de l'éducation, 30(1), 143-162.

Conseil supérieur de l'éducation. (1999). Pour une meilleure réussite scolaire des garçons et des filles. Panorama. Récupéré le 19 septembre 2011 de http://www.cse.gouv.qc.ca/f/pub/panorama/99-11/gar-fil.htm

Cornalba, V. (2009). Je, Net et Tchatche. Adolescence, 4(70), 971-982.

Crotty, M. (1998). The Foundations of Social Research. Thousand Oaks, CA : Sage Publications. 
Dalpé, V., Giroux, C., Lefebvre, F. et St-Pierre, M.-C. (2010). Composantes de la lecture et de l'écriture. Dans M.-C. St-Pierre., V. Dalpé., P. Lefebvre et C. Giroux (Dir.), Difficultés de lecture et d'écriture. Prévention et évaluation orthographique auprès des jeunes (pp. 29-45). Québec: Presses de l'Université Laval.

David, J. et Goncalves, H. (2007). L'écriture électronique, une menace pour la maîtrise de la langue? Le français aujourd'hui, 156, 39-48.

Debeurme, G. et Lamarche, M. (2001). Thématiques: difficultés d'apprentissage-écriture. Site de l'adaptation scolaire et sociale de la langue française.

Deschênes, A. J. (1991). La lecture: une activité stratégique. Dans Les Entretiens Nathan sur la lecture, Actes 1 (pp. 29-49). Paris : Nathan.

Dion-Viens, D. (2006). Les jeunes branchés: «Slt, qus tu fè ?» Le Soleil. Récupéré le 13 novembre 2009 de http://archives.infobourg.com/sections/actualite/actualite. php?id=10887

Dion-Viens, D. (2008). Le «texto »n'est pas une menace pour le français. Le Soleil. Récupéré le 13 novembre 2009 de http ://www.cyberpresse.ca/vivre/200809/08/ 01-652751-le-texto-nest-pas-une-menace-pour-le-francais.php

Doly, A. M. (1997). Métacognition et médiation à l'école. Dans M. Grangeat et P. Meirieu (Dir.), La métacognition, une aide au travail des élèves (pp. 17-61). Paris: ESF éditeur.

Doudin, P. A. et Martin, D. (1998). Métacognition et formation des enseignants. Dans L. Lafortune, P. Mongeau et R. Pallascio (Dir.), Métacognition et compétences réflexives (pp. 23-46). Montréal : les Éditions Logiques.

Doyon, F. (2007). Le clavardage, massacre de la langue ou renaissance linguistique? Le Devoir. Récupéré le 13 novembre 2009 de http :/www.ledevoir.com/societe/ education/129491/le-clavardage-massacre-de-la-langue-ou-renaissancelinguistique

Faverjon, J. (2003). Clavardage = clavier + bavardage. Récupéré le 28 février 2010 de http ://www.europeanmediaculture.org/fileadmin/bibliothek/francais/faverjon_cla varder/faverjon_clavarder.html

Fayol, M. (1991). La production d'écrits et la psychologie cognitive. Le français aujourd'hui, 93, 21-24.

Filatova, K. L. (2007) La construction de l'illusion: mécanismes linguistiques et cognitifs qui assurent la compréhension métaphorique du clavardage. Paris: L'Harmattan.

Flavell, J. H. (1985). Cognitive development (2 2 éd.). Englewood Cliffs, NJ : Prentice-Hall. 
Fuks, H., Pereira de Lucena, C. J. et Pimentel, M. (2006). R-U-Typing Me? Evolving a Chat Tool to Increase Understanding in Learning Activities. International Society of the Learning Sciences, 1(1), 117-142.

Gadet, F. (2003). La variation et le français dans l'espace social, régional et international. Dans M. Yaguello (Dir.), Le Grand Livre de la Langue Française (pp. 91-156). Paris : Seuil.

Gagné, E. D. (1985). The cognitive psychology of school learning. Boston : Little, Brown and Company.

Gagné, R. M. (1970). The conditions of learning. New-York : Holt, Rinehart and Winston.

Goudailler, J.-P. (2007). Français contemporain des cités: langue en miroir, langue du refus. Adolescence, 1(59), 119-124.

Grangeat, M. (1997). La métacognition, un enjeu pour autonomisation. Dans M. Grangeat et P. Meirieu (Dir.), La métacognition, une aide au travail des élèves (pp. 95129). Paris : ESF éditeur.

Gustin, M., Klein, G. et Rosenberg, S. (1999). Projet de recherche par des étudiants de l'université de Miami dans le cadre du cours Social Psychology of cyberspace. Tapscott says, Raised of the Net: The Effects of Computerization on the Next Generation. Récupéré le 5 septembre 2010 de http ://miavx 1.muohio.edu/psybersite/cyberspace/n-gen/tapscott.htx

Hagège, C. (2006). Combat pour le français au nom de la diversité des langues et des cultures. Paris : Odile Jacob.

Hickmann, M., Hendriks, H. et Roland, T. (1998). Référence spatiale dans les écrits d'enfants français : perspective inter-langues. Langue française, 118, 104-123.

Howell, D. (2008). Méthodes statistiques en sciences humaines. Bruxelles: De Boeck Université.

Jaffré, J. P. (2003). L'écriture et les nouvelles technologies: ce que les unes nous apprennent des autres. Actes des Quatrièmes Rencontres Réseaux Humains / Réseaux Technologiques (pp. 81-86). Poitiers: Actes et Rapports pour l'éducation. Récupéré le 15 novembre 2009 de http ://rhrt.edel.univpoitiers.fr/document.php?id=551

Jia, G. et Aaronson, D. (1999) Age Differences in Second Language Acquisition: The Dominant Language Switch and Maintenance Hypothesis. Dans A. Greenhill, $\mathrm{H}$. Littlefield et C. Tano (Dir.), Proceedings of the $23^{\text {rd }}$ Annual Boston University Conference on Language Development (pp. 301-312). Somerville, M.A.: Cascadilla Press. 
Karsenti, T. et Savoie-Zajc, L. (2004). La recherche en éducation (2 ${ }^{\mathrm{e}}$ éd.). Sherbrooke: Éditions du CRP.

Kern, R. (1995). Restructuring Classroom Interaction with Networked Computers : Effects on Quantity and Characteristics of Language Production. Modern Langage Journal, 79, 457-476.

Labbé, H. et Marcoccia, M. (2005). Communication numérique et continuité des genres : l'exemple du courrier électronique. Texto ! X(3). Récupéré le 18 octobre 2010 de http ://www.revue-texto.net/Inedits/Labbe-Marcoccia.html

Lafontaine, M., Blouin-Bradette, M., Cantin-Fontaine, M. et Fortier, M. M. (2005). Langue et ordinateur: le clavardage et le français font-ils bon ménage? Québec français, $137,64-66$.

La Haye, D. (2006). Donner sa langue au «ch@t». Le Droit. Récupéré le 13 novembre 2009 de http ://technaute.cyberpresse.ca/nouvelles/internet/200603/20/01-15547donner-sa-langue-au-cht.php

Léger, D. (2006). Le clavardage : danger linguistique ou avantage social? Le clavardeur, le journal des élèves du secondaire. Récupéré le 13 novembre 2009 de http ://www.leclavardeur.com/no_anterieurs_journal.asp?cleN $1=100 \& c l e N 2=200$ $7021308534771596 \&$ cleN3 $=2007021308551283388$

Levesque, J.-Y., Lavoie, N. et Chénard, M. (MELS : 2002-2007). Recherche action collaborative pour la réussite scolaire des garçons en lecture et en écriture. Rimouski : Université du Québec à Rimouski.

Lieury, A. (2005). Psychologie cognitive en 35 fiches, des principes aux applications. Paris: Dunod.

Lindsay, P. H. et Norman, D. A. (1980). Traitement de l'information et comportement humain, une introduction à la psychologie. Montréal : Études vivantes.

Lioger, E. (2002). Quelles approches théoriques pour la description du français parlé par les jeunes des cités. La linguistique, 38(1), 41-52.

Luzzati, D. (1991). Oralité et interactivité dans l'écrit Minitel. Langue française, 89(1) 99109.

Marcoccia, M. (2003). La communication médiatisée par ordinateur : problèmes de genres et de typologie. Communication présentée aux Journées d'études: les genres de l'oral. Lyon : Laboratoire Tech-CICO, Université de Technologie de Troyes.

Mercier, L. (2002). Le français, une langue qui varie selon les contextes. Dans C. Verreault., L. Mercier et M. Lavoie (Dir.), Le français, une langue à apprivoiser. Textes des conférences prononcées au Musée de la civilisation (Québec, 2000- 
2001) dans le cadre de l'exposition Une grande langue: le français dans tous ses états (pp. 41-60). Québec: Les Presses de l'Université Laval.

Ministère de l'Éducation du Québec, Gouvernement du Québec. (2003). Résultats obtenus en 2001 par les élèves québécois de dix ans. Direction de la sanction des études, Programme international de recherche en lecture scolaire (PIRLS).

Ministère de l'Éducation, du Loisir et du Sport, Gouvernement du Québec. (2006). Indicateurs de l'éducation, Édition 2006. Secteur de l'information et des communications.

Morgenstern, A. (2009). L'enfant dans la langue. Paris : Presses Sorbonne Nouvelle.

Nadeau, M. (1995). Propositions pour améliorer le transfert des connaissances en orthographe grammaticale. Québec français, 99, 35-38.

Negretti, R. (1999). Web-Based Activities and SLA : a Conversation Analysis Research Approach. Language Learning and Technology, 3(1), 75-87.

Nicolas, S. (2003). La psychologie cognitive. Paris : Armand Colin.

Nolet, M. (2006). Bon ou mauvais le clavardage? Récupéré le 19 décembre 2010 du site du Réseau pour le développement des compétences par l'intégration des technologies : http ://recit.nordtic.net/spip.php?article37

Office québécois de la langue française. (2002). Bibliothèque virtuelle. Récupéré le 26 septembre 2009 de http://www.olf.gouv.qc.ca/RESSOURCES/ bibliotheque/dictionnaires/Internet/fiches/8392463.html

Ouellon, C. (2010). La maîtrise de la langue d'enseignement - entre crispation et espoir. Communication présentée à la Rencontre des gestionnaires de l'éducation, Laval, Québec.

Panckhurst, R. (1999). Analyse linguistique assistée par ordinateur du courriel. Dans J. Anis (Dir.), Internet, communication et langue française (pp. 55-70). Paris: Hermès.

Paré, G. (2001). Rapport Bourgogne. Génération Internet: la prochaine grande génération. Montréal: Centre interuniversitaire de recherche en analyse des organisations.

Perraudeau, M. (1996). Les méthodes cognitives, apprendre autrement à l'école. Paris: Armand Colin.

Portelance, L. (1998). Enseigner en vue de développer la compétence métacognitive: comment et pourquoi? Dans L. Lafortune, P. Mongeau et R. Pallascio (Dir.), 
Métacognition et compétences réflexives (pp. 47-65). Montréal : les Éditions Logiques.

Préfontaine, C. et Lebrun, M. (1992). La lecture et l'écriture, enseignement et apprentissage. Montréal : les Éditions Logiques.

Radio-Canada. (2007). Êtes-vous indifférent? Récupéré le 18 août 2010 de http ://www.radio-canada.ca/nouvelles/societe/2007/02/02/003-clavardage-code. shtml

Réseau de télématique scolaire québécois. (2010). Clavardage et pédagogie. Récupéré le 22 novembre 2009 de http ://rtsq.qc.ca/communiquer/rencontres_virtuelles.htm

Réseau Éducation-Médias. (2005). Jeunes Canadiens dans un monde branché (JCMB). Récupéré le 22 octobre 2009 de http://www.media-awareness.ca/francais/ recherche/JCMB/index.cfm.

Réseau Éducation-Médias. (2009). Contrer la cyberintimidation. Récupéré le 22 octobre 2009 de http ://www.mediaawareness.ca/francais/ressources/projets_ speciaux/toile_ressources/Contrer_cyberintimidation.cfm

Robin, R. (2000). «Le texte cyborg ». Études françaises, 36(2), 11-38.

Roy, G. R., Lafontaine, L., Boudreau, G. et Viau, R. (1992). Vers un triple regard sur le français écrit des étudiants de collèges et d'universités. Sherbrooke: Éditions du CRP.

Sarrasin, L. (2010). Ordinateur et nouvelles formes d'écriture: en quoi cela change nos vies. Récupéré le 25 mai 2010 du site du Récit pour le service national du domaine des langues : http ://www.recit.qc.ca/spip.php?article86

Schneider, W. (1986). The Role of Conceptual Knowledge and Metamemory in the Development of Organizational Process in Memory. Journal of Experimental Child Development, 41, 218-236.

Smith, K. L. (1990). Collaborative and Interactive Writing for Increasing Communication Skills. Hispania, 73, 77-87.

Sousa, D. A. (2002). Un cerveau pour apprendre. Comment rendre le processus enseignement-apprentissage plus efficace. Montréal : Chenelière/McGraw-Hill.

Sternberg, R. (2007). Manuel de psychologie cognitive, du laboratoire à la vie quotidienne. Bruxelles : De Boeck Université.

Tapscott, Don. (1998). The Net Generation and the School. Récupéré le 12 février 2011 du site de la Milken Family Foundation: http ://www.mff.org/edtech/article.taf?_function=detail\&Content_uidl $=109$. 
Tardif, J. (1992). Pour un enseignement stratégique, l'apport de la psychologie cognitive. Montréal : les Éditions Logiques.

Totassian, A. (2008). Typologie des procédés scripturaux des salons de clavardage en français chez les adolescents et les adultes. Dans J. Durant, B. Habert et B. Laks (Dir.), Actes du premier congrès mondial de linguistique française (pp. 2337-2353). Paris : Institut de Linguistique Française.

Touzin, M. (2004). La rééducation des troubles spécifiques d'acquisition du langage écrit. Dans T. Rousseau (Dir.), Les Approches Thérapeutiques en Orthophonie, tome 2. Prise en charge orthophoniques des troubles du langage écrit (pp. 65-82). Isbergues, France : Ortho éditions.

Tudini, V. (2003). Éléments conversationnels du clavardage: un entrainement à l'expression orale pour les apprenants de langue à distance? Apprentissage des langues et systèmes d'information et de communication, 6(2), 63-81.

Varnhagen, C., McFall, G., Pugh, N., Routhledge, L. et Sumida-MacDonald, H. (2009). Lol : New Language and Spelling in Instant Messaging. Reading and Writing, 23(6), 719-733.

Warschauer, M. (1996). Comparing Face-to-Face and Electronic Communication in the Second Language Classroom, CALICO Journal, 13, 7-26.

Watrelot, M. (2008). De la France au Québec : l'écriture dans tous ses états. Colloque international. IUFM Poitou-Charentes, site de Poitiers.

Wilkinson, A. C. (1982). Partial Knowledge and Self-Correction : Developmental Studies of a Quantitative Concept. Developmental Psychology, 18(6), 876-893.

Wong, B. Y. L. (1991). The relevance of metacognition to learning disabilities. Dans B. Y. L. Wong (Dir.), Learning about learning disabilities (pp. 231-258). New-York: Academic Press. 

